\title{
$(2+1)$ EINSTEIN SPACETIMES OF FINITE TYPE
}

\author{
RICCARDO BENEDETTI AND FRANCESCO BONSANTE \\ Dipartimento di Matematica, Largo B. Pontecorvo 5, Pisa, ITALY \\ benedett@dm.unipi.it \\ Scuola Normale Superiore, Pisa, ITALY \\ f.bonsante@sns.it
}

\section{CONTEnts}

1. Introduction 2

2. 3-dimensional gravity 4

2.1. General background 4

2.2. (2+1)-spacetimes

3. The space $\mathcal{M L}(S)$

3.1. The Teichmüller space $\widetilde{\mathcal{T}}(S)$

3.2. The convex-core map 8

3.3. The space of measured geodesic laminations 14

3.4. The sub-space $\mathcal{M L}_{\mathfrak{c}}(S)$

3.5. Enhanced bundle $\mathcal{M L}_{\mathfrak{c}}(S)^{\#}$ and measure spectrum 17

3.6. Grafting, bending, earthquakes 22

4. Wick rotation-rescaling theory $\quad 27$

4.1. Cosmological time 28

4.2. Grafting and Lorentzian grafting $\quad 29$

4.3. Wick rotation - rescaling set up 32

4.4. Flat spacetimes classification 33

4.5. Wick rotation: flat Lorentzian vs hyperbolic geometry 37

4.6. Flat vs de Sitter Lorentzian geometry 43

4.7. Flat vs Anti de Sitter Lorentzian geometry 44

5. Causal AdS spacetimes, earthquakes and black holes 52

5.1. On holonomy pregnancy 52

5.2. Canonical causal AdS spacetimes with prescribed holonomy 53

5.3. AdS bending and Earthquake Theorems

5.4. Convex core of $\Omega(h)$ and black hole $\quad 55$

5.5. (Broken) T-symmetry 58

6. Including particles 59

6.1. Maximal globally hyperbolic spacetimes with particles 61

6.2. Earthquakes on hyperbolic cone surfaces $\quad 62$

References 


\section{InTRODUCTION}

A surface $S$ is said to be of finite type if:

(1) it is of the form

$$
S=\hat{S} \backslash V
$$

where $(\hat{S}, V)$ is a compact closed oriented surface of genus $g \geq 0$, with a set of $r \geq 0$ marked points $V=\left\{p_{1}, \cdots, p_{r}\right\}$;

(2) the fundamental group of $S$ is non Abelian, equivalently $2-2 g-r<0$.

The main aim of this survey is to widely describe, for every $S$ of finite type, and for every $\kappa=0, \pm 1$, the geometry of 3-dimensional maximal globally hyperbolic Lorentzian spacetimes of constant curvature $\kappa$, that contain a complete Cauchy surface homeomorphic to $S$. We call them generically Einstein $M G H$ spacetimes of finite type. The (3-dimensional) general relativity background will be briefly recalled in Section 2 . These spacetimes are supported by the product $S \times \mathbb{R}$; considered up to Lorentzian isometry homotopic to the identity of $S \times \mathbb{R}$, they form, for every $\kappa$, a Teichmüller-like space denoted

$$
\mathcal{M G H}_{\kappa}(S)
$$

Clearly these notions make sense also if $S$ is not necessarily of finite type. In the monograph [10] we have developed a canonical Wick rotation-rescaling theory on such general $M G H$ spacetimes. It is easy to see that $\mathcal{M G H}_{\kappa}(S) \neq \emptyset$ for every $\kappa$, if and only if the open disk $D^{2}$ is the universal covering of $S$. In [10] we have actually analyzed $\mathcal{M H \mathcal { G }}_{\kappa}\left(D^{2}\right)$, by developing also an equivariant version of the theory, with respect to the action of any deck transformation group. WR-rescaling theory includes a wide generalization of Mess classification [41] (completed by Scannell [45] for $\kappa=1$ ) of $M G H$ spacetimes with compact Cauchy surfaces $(i . e . V=\emptyset)$. Moreover, it establishes explicit geometric correlations between spacetimes of different curvatures, or between spacetimes and complex projective structures on $S$. In particular, this gives a clear geometric explanation of the occurrence of a certain "universal" parameter space

$$
\mathcal{M L}(S)
$$

shared by all $\mathcal{M G H}_{\kappa}(S), \kappa=0, \pm 1$, and by $\mathcal{P}(S)$, the Teichmüller-like space of complex projective structures on $S$.

A large part of this survey just reports on such a theory, by specializing it to $S$ of finite type. This class is large enough to display the main features of the theory; on the other hand, spacetimes of finite type are possibly easier to figure out than completely general ones. In fact we will spell out several specific statements that are quite implicit in the general treatment given in [10]. Hence the present paper should be an actual complement to that monograph. Moreover, there are in this case direct relations between $\mathcal{M L}(S)$ and more familiar Teichmüller spaces of hyperbolic structures on $S$ and, to some extent, with the corresponding tangent bundles (see Section 3). For example, when $S$ is compact $\mathcal{M L}(S)$ coincides with the (topologically trivialized) bundle $\mathcal{T}_{g} \times \mathcal{M} \mathcal{L}_{g}$ of measured geodesic laminations on hyperbolic structures on $S$; in general we will deal with hyperbolic structures $F$ on $S$ whose completions $F^{\mathcal{C}}$ have (non necessarily compact) geodesic boundary, and with a kind of measured geodesic laminations $\lambda$ on $F^{\mathcal{C}}$. In fact, another goal should be to convince a reader familiar with 
such topics of hyperbolic geometry, that not only this provides some important tools for studying Einstein spacetimes; in the reverse direction, via Lorentzian geometry we get a new insight into several fundamental hyperbolic constructions such as grafting, (3-dimensional hyperbolic) bending and earthquakes along laminations. To support this claim we just mention here the "AdS proof" of Thurston Earthquakes Theorem for hyperbolic structures on compact surfaces $S$, that Mess obtained in [41] as a byproduct of his classification of spacetimes in $\mathcal{M G H}_{-1}(S)$. An AdS look at earthquakes theory beyond the compact case will be a theme of this paper (see Section 5).

Finally, we note that spacetimes of finite type occur (via canonical Wick rotation) as "ending spacetimes" of geometrically finite hyperbolic 3-manifolds, which furnish basic examples for a bordism category supporting $(2+1)$ QFT pertinent to 3-dimensional gravity (see Section 1.11 of [10], and [8]).

In Section 5 we focus the AdS case that displays the richer phenomenology, mostly referring (besides [10]) to [6] $(1,2)$ and [19]. In particular we will describe the common maximal causal extension $\Omega(h)$ of the $M G H$ spacetimes of finite type that share a given AdS holonomy $h$. We will see that $\Omega(h)$ is still supported by the product $S \times \mathbb{R}$ but it is not in general globally hyperbolic. This is a particularly interesting case, because we can detect a specific one among the maximal globally hyperbolic spacetimes contained in $\Omega(h)$ that can be truly considered as a black hole. The analysis of the causal extension is also important to achieve a proof of the Earthquake Theorem.

Finally, in Section [6 we will outline (by following [11](2) and mostly [18]) how the Wick rotation - rescaling theory (partially) extends to $M G H$ spacetimes of finite type that include world lines of "particles" (i.e inextensible time-like lines of space-like conical singularities).

We stress that this paper is not intended to be exhaustive of the subject. We have made a few partial and subjective choices, organized around our favorite Wick rotationrescaling view point. Nevertheless, we hope that this would be enough to show that 3-dimensional gravity is a fairly non-trivial and beautiful "toy model". In particular, we have neglect a classical analytic approach to the classification of constant curvature MGH spacetimes in terms of solutions of the Gauss-Codazzi equation at a Cauchy surface, possibly imposing some supplementary conditions to such solutions, that translate some geometric property of the embedding of $S$ as Cauchy surface (see also Section 2). A widely studied possibility requires that the surface has constant mean curvature (see for instance [42, 3, 7, 36]). At least for compact $S$, the classical Teichmüller space of conformal structures on $S$, with its complex cotangent bundle arises in this way towards the classification. This approach also selects a distinguished $C M C$ global time on $M G H$ spacetimes, that basically coincides with the mean curvature of its level surfaces.

Wick rotation-rescaling theory is based on a rather different more geometric approach, initiated by Mess in [41]. It turns out that a key ingredient is another canonical time, the so called cosmological time. Every $M G H$ spacetime is in a sense determined by the "asymptotic states" of the corresponding level surfaces, rather than the embedding data of some Cauchy surface. The Wick rotation-rescaling mechanism is ultimately based on the fact that $M G H$ spacetimes (of different curvatures) can be associated in 
such a way that the intrinsic geometry of these level surfaces does not depend on the curvature, up to some scale factor.

\section{3-DIMENSIONAL GRAVITY}

2.1. General background. For the basic notions of global Lorentzian geometry and causality we refer for instance to [9, 31].

A $(n+1)$ spacetime consists of a $(n+1)$-manifold $M$ equipped with a Lorentzian metric $h$ and with a time orientation, so that the causal past/future of every event $p \in(M, h)$ is determined. We also stipulate that $M$ is oriented.

Roughly speaking, the general problem of gravity can be stated as follows. Given a $(n+1)$-manifold $M$, a symmetric $(0,2)$-Tensor $T$ on $M$ and a constant $\Lambda$ (called the cosmological constant), find out all spacetimes $(M, h)$ such that:

(a) The metric $h$ satisfies the Einstein equation

$$
\operatorname{Ric}_{h}+\left(\Lambda-1 / 2 R_{h}\right) h=T
$$

where $R i c_{h}$ is the Ricci tensor of $h, R_{h}$ is the scalar curvature.

(b) The global causal structure of $(M, h)$ satisfies determined conditions.

These spacetime structures are considered up to diffeomorphism of $M$ that preserves the tensor $T$. Both the features of the tensor $T$ and of the causality conditions are determined by physical (even logical) considerations. Normally they also impose some constraints on the topology of $M$. Requirements in (a) and (b) are basically of independent nature.

The pure gravity case is when $T=0$. In such a case the solutions of Einstein equation coincide with the so called Einstein metrics: Ric $_{h}=\frac{2}{n-1} \Lambda h$.

The basic causality condition is that $(M, h)$ is chronological (causal), that is it does not contain any closed timelike (causal) curve $c$. A curve is said timelike (causal) if the velocity field $v(t)$ along $c$ is timelike (causal): $h(v(t), v(t))<0(h(v(t), v(t)) \leq 0)$.

The strongest causality condition is that $(M, h)$ contains a Cauchy surface $S$; this means that $S$ is a spacelike hypersurface of $M$ (the restriction of $h$ to $S$ is Riemannian), such that every causal inextensible line of $(M, h)$ intersects $S$ exactly once. In such a case we say that $(M, h)$ is globally hyperbolic. If $(M, h)$ is globally hyperbolic the $M$ turns to be a product manifold $M \cong S \times \mathbb{R}$ so that (up to some diffeomorphisms of $M$ ) the Cauchy surface $S$ coincides with $S \times\{0\}$, and every slice $S \times\{t\}$ is $h$-spacelike (indeed we can also require that every such a slice eventually is a Cauchy surface of $(M, h)$ ). Such a picture is coherent with the intuitive idea of a space evolving in time. Globally hyperbolic spacetimes naturally arise as dependence domains $\left(D(S), h_{\mid D(S)}\right)$ of spacelike hypersurfaces $S$ in arbitrary spacetimes $(M, h) ; S$ turns to be a Cauchy surface of $D(S)$. Hence globally hyperbolic spacetimes make a fundamental sector of gravity theory.

2.2. $(2+1)$-spacetimes. 3D gravity is much simpler (but non trivial) because in three dimensions the Riemann tensor is determined by the Ricci tensor. In particular 3D Einstein metrics actually have constant (sectional) curvature. The sign of the curvature 
coincides with the sign of the cosmological constant. We will be mainly concerned by $(2+1)$ globally hyperbolic Einstein spacetimes $(M, h)$ (i.e. of constant curvature $\kappa)$. We recall two possible ways to study such spacetimes. The first analytic one is based on the important fact that the germ of the metric $h$ at a Cauchy surface $S$ determines, in a sense that we will make precise, the whole spacetime. This leads to consider the pairs $(g, b)$ of a Riemannian metric, $g$, on the surface $S$ and a $g$-symmetric endomorphism, $b$, of $T S$, that verify the Gauss-Codazzi equation

$$
\begin{aligned}
& d^{\nabla} b=0 \\
& \operatorname{det} b=\kappa-\kappa_{g}
\end{aligned}
$$

where $d^{\nabla}$ is the differential with respect to the Levi-Civita connection of $g, \kappa$ is a constant and $\kappa_{g}$ is the Gauss curvature of $g$.

It is possible to associate to such a pair $(g, b)$ a Lorentzian metric $h$ on $M=S \times \mathbb{R}$ of constant curvature $\kappa$, such that $S=S \times\{0\}$ is a Cauchy surface, the first fundamental form of $S$ in $(M, h)$ is $g$ and the shape operator is $b$ (recall that the shape operator of a spacelike surface $F$ in a Lorentzian or Riemannian manifold $M$ is the endomorphism of $T F$ that coincides with the covariant derivative of the normal field of $F$ in $M)$. A priori $(g, b)$ determines only the germ of $h$ around $S \times\{0\}$. On the other hand, it is proved in 22] that there exists a unique (up to isometries) such a globally hyperbolic spacetime $\left(M_{\max }, h_{\max }\right)$ that is maximal in the following sense:

Given any globally hyperbolic spacetime $(M, h)$ as above, there exists an isometric embedding of $(M, h) \rightarrow\left(M_{\max }, h_{\max }\right)$ that is the identity on $S \times\{0\}$ (and preserves the orientations).

At a first sight, this definition of "maximality" involves the choice of a Cauchy surface (i.e. $S \times\{0\}$ ). On the other hand, one can see that it is equivalent to the following one:

Every isometric embedding of $\left(M_{\max }, h_{\max }\right)$ into an Einstein spacetime $(N, k)$ that sends any Cauchy surface of $M_{\max }$ onto a Cauchy surface of $N$ actually is a global isometry.

This last property gives a good definition of the class of maximal globally hyperbolic $(M G H)$ Einstein spacetimes, that makes intrinsic sense, not depending on the analytic approach we are outlining. It is reasonable to restrict to this class in order to get a classification.

Continuing with the analytic approach, a well-defined map eventually associates to every pair $(g, b)$ as above the (isotopy class of the) maximal globally hyperbolic spacetime $\left(M_{\max }, h_{\max }\right)_{(g, b)}$. Such map is surjective, but not injective. In fact it establishes a bijective correspondence between pairs $(g, b)$ and spacetimes with a marked Cauchy surface. To get rid of this excess of degrees of freedom, some additional condition on $(g, b)$ has to be imposed, possibly translating some geometric property of the Cauchy surface embedding. A widely investigated possibility consists in requiring that the trace of $b$ is constant, that is, $S \times\{0\}$ is a surface of constant mean curvature.

The second geometric approach makes use of the $(X, G)$-structure technology. Indeed any $(2+1)$ Einstein spacetime $M$ is a $\left(\mathbb{X}_{\kappa}, \operatorname{Isom}\left(\mathbb{X}_{\kappa}\right)\right)$-manifold, where $\mathbb{X}_{\kappa}$ is a suitable isotropic model of constant curvature $\kappa$. 
Denote $\tilde{M}$ an universal covering of $M$. A very general "analytic continuation" procedure allows to associate to every $(X, G)$-manifold, $M$, a compatible couple $(d, h)$, where $d$ is a developing map, that is a local isomorphism

$$
d: \tilde{M} \rightarrow X
$$

and $h$ is a holonomy representation

$$
h: \pi_{1}(M) \rightarrow G
$$

such that $\left(\pi_{1}\right.$-equivariance):

$$
d(\gamma x)=h(\gamma) d(x)
$$

(where $\pi_{1}(M)$ is identified to the covering transformation group of $\tilde{M}$ ). The developing map is determined up to post-composition by any element of $G$, whereas the holonomy is determined up to conjugation by the same element.

Conversely a local diffeomorphism $d: \tilde{M} \rightarrow X$ equivariant with respect to a representation $h: \pi_{1}(M) \rightarrow G$ produces a well-defined $(X, G)$-structure on $M$.

In this paper we will mainly focus on this second geometric approach. For this reason we will briefly recall the principal features of the isotropic models of constant curvature $\kappa$, that we will normalize to be $\kappa=0,1,-1$.

Minkowski space. The isotropic model of flat spacetimes, $\mathbb{X}_{0}$, is the Minkowski space, that is $\mathbb{R}^{3}$ equipped with the flat metric $-\mathrm{d} x_{0}^{2}+\mathrm{d} x_{1}^{2}+\mathrm{d} x_{2}^{2}$. Isometries of $\mathbb{X}_{0}$ are affine transformations whose linear part preserves the Minkowski product (that is $\left.\operatorname{Isom}\left(\mathbb{X}_{0}\right)=S O(2,1) \rtimes \mathbb{R}^{3}\right)$. We consider the time-orientation on $\mathbb{X}_{0}$ such that the $x_{0^{-}}$ component of future-directed timelike vectors is positive. The set of future directed unit timelike vectors is a hypersurface of $\mathbb{X}_{0}$ that inherits from $\mathbb{X}_{0}$ a Riemannian metric. This is the hyperboloid model of the hyperbolic plane $\mathbb{H}^{2}$. The isometric action of $S O^{+}(2,1)$ on it induces an identification between $S O^{+}(2,1)$ and $\operatorname{PSL}(2, \mathbb{R}) \cong \operatorname{Isom}^{+}\left(\mathbb{H}^{2}\right)$ (by using also the Poincaré half-plane model of $\mathbb{H}^{2}$ ). The main advantage of the hyperboloid model is that geodesics are just obtained by intersecting $\mathbb{H}^{2}$ with timelike planes. In particular the duality between linear planes and linear straight lines given by the orthogonality relation induces an identification between the set of geodesics of $\mathbb{H}^{2}$ and the set of un-oriented spacelike directions of $\mathbb{X}_{0}$. The projection of $\mathbb{H}^{2}$ in the projective plane $\mathbb{P}\left(\mathbb{R}^{3}\right)$ is injective and the image is the set of timelike directions. Notice that in this projective (Klein) model geodesics are just projective lines. Moreover, the set of lightlike directions is the boundary of $\mathbb{H}^{2}$ and the end-points of a geodesic $l$ in $\mathbb{H}^{2}$ are the two lightlike directions contained in the plane of $\mathbb{X}_{0}$ containing $l$.

By using the 4-dimensional Minkowski space, in a similar way we get the different models of the hyperbolic space $\mathbb{H}^{3}$.

De Sitter space. The set of unit spacelike vectors in 4-dimensional Minkowski space, is a Lorentzian submanifold of constant curvature 1. It is called the de Sitter spacetime and will be denoted by $\hat{\mathbb{X}}_{1}$. The isometric action of $S O(3,1)$ on $\hat{\mathbb{X}}_{1}$ shows that this model is isotropic and that its isometry group coincides with $S O(3,1)$. Also in this model geodesic are obtained by intersecting $\hat{\mathbb{X}}_{1}$ with a linear plane of the Minkowski space. In particular spacelike geodesics are closed with length equal to $2 \pi$, whereas timelike geodesics are embedded lines with infinite length. 
It is often convenient to consider the projection of $\hat{\mathbb{X}}_{1}$ into the projective space $\mathbb{P}\left(\mathbb{R}^{4}\right)$. Notice that the image, $\mathbb{X}_{1}$, is the set of spacelike directions, that is, it is the exterior of $\mathbb{H}^{3}$ into $\mathbb{P}\left(\mathbb{R}^{4}\right)$. Clearly the projection $\hat{\mathbb{X}}_{1} \rightarrow \mathbb{X}_{1}$ is a 2 -to-1 covering, so $\mathbb{X}_{1}$ is not simply connected. On the other hand, since $\mathbb{X}_{1}=\hat{\mathbb{X}}_{1} /\{ \pm I d\}$, and $\{ \pm I d\}$ is the center of $S O(3,1)$, also $\mathbb{X}_{1}$ is an isotropic model of the de Sitter geometry. Its isometry group if $S O(3,1) /\{ \pm I d\} \cong S O^{+}(3,1)$. An advantage in using this model is that $\mathbb{X}_{1}$ and $\mathbb{H}^{3}$ share the same asymptotic boundary and their isometry groups actually coincide. By means of the duality between geodesic planes of $\mathbb{H}^{3}$ and spacelike directions of Minkowski space, $\mathbb{X}_{1}$ can be regarded as the set of un-oriented geodesic planes of $\mathbb{H}^{3}$.

Anti de Sitter space. Consider on $\mathbb{R}^{4}$ a scalar product $\eta$ with signature $(2,2)$, then the set of unit timelike vectors is a Lorentzian submanifold $\hat{\mathbb{X}}_{-1}$ of constant curvature -1 . Let $\mathbb{R}^{4}$ be identified with the set of $2 \times 2$ matrices, and consider the form $\eta$ such that $\eta(X, X)=-\operatorname{det} X$. The signature of $\eta$ is $(2,2)$, so an explicit model of $\hat{\mathbb{X}}_{-1}$ is $S L(2, \mathbb{R})$ equipped with its Killing form. The isometric action of $S L(2, \mathbb{R}) \times S L(2, \mathbb{R})$ on $S L(2, \mathbb{R})$ by left and right multiplication shows that $\hat{\mathbb{X}}_{-1}$ is isotropic and that its isometry group is $S L(2, \mathbb{R}) \times S L(2, \mathbb{R}) /(-I d,-I d)$.

As in the previous case the projection of $\hat{\mathbb{X}}_{-1}$ into the projective space $\mathbb{P}\left(\mathbb{R}^{4}\right)$, is a 2-to- 1 covering map on a open set $\mathbb{X}_{-1}$ of $\mathbb{P}\left(\mathbb{R}^{4}\right)$ that is $\operatorname{PSL}(2, \mathbb{R})$. Since the covering transformations of $\hat{\mathbb{X}}_{-1} \rightarrow \mathbb{X}_{-1}$ are $\pm I d$ it follows that $\mathbb{X}_{-1}$ inherits from $\hat{\mathbb{X}}_{-1}$ an isotropic Lorentzian metric of constant curvature -1 . The isometry group of $\mathbb{X}_{-1}$ turns to be $P S L(2, \mathbb{R}) \times P S L(2, \mathbb{R})$.

Topologically $\mathbb{X}_{-1}$ is a solid torus and its boundary in $\mathbb{P}\left(\mathbb{R}^{4}\right)$ can be identified with the projective classes of rank 1 matrices. The Segre embedding produces a double foliation on $\partial \mathbb{X}_{-1}$ by projective lines (actually it induces a product structure $\partial \mathbb{X}_{-1}=$ $\left.\mathbb{P}^{1} \times \mathbb{P}^{1}\right)$. Isometries of $\mathbb{X}_{-1}$ extends on the boundary: the left multiplication preserves the leaves of the left foliation, whereas right multiplication preserves the leaves of the right foliation. Notice that the product structure on the boundary can be regarded as a conformal Lorentzian structure.

Geodesics and geodesic planes of $\mathbb{X}_{-1}$ are the intersection of $\mathbb{X}_{-1}$ with projective lines and projective planes of $\mathbb{P}\left(\mathbb{R}^{4}\right)$. In particular projective lines contained in $\mathbb{X}_{-1}$ are timelike geodesic of length $\pi$, projective lines tangent to the boundary are lightlike lines and projective lines intersecting the boundary in two points are spacelike geodesic of infinite length. Notice that spacelike geodesics are determined by their end-points on the boundary. Conversely given two points on the boundary that do not lie on the same left nor right leaf, there exists a unique spacelike geodesic connecting them.

Projective planes intersecting $\mathbb{X}_{-1}$ along compression disks are spacelike planes and turn to be isometric to $\mathbb{H}^{2}$. Points of $\mathbb{X}_{-1}$ bijectively corresponds to spacelike planes via the duality induced by $\eta$ between points of $\mathbb{P}\left(\mathbb{R}^{4}\right)$ and projective planes. There is a geometric interpretation of such a duality: given a point $x \in \mathbb{X}_{-1}$, its dual plane $P(x)$ is the set of points at distance $\pi / 2$ from $p$ along some timelike geodesic. Conversely given a spacelike plane, its normal geodesics intersect at the dual point of the plane. Given a spacelike geodesic line $l$, the points $x$ such that $l \subset P(x)$ form another spacelike line $l^{*}$, that is the dual geodesic of $l$. 


\section{The SPACE $\mathcal{M L}(S)$}

This section is entirely settled in the framework of (2-dimensional) hyperbolic geometry, and several facts that we are going to recall are well-known. However, we will give later a new insight (if not an outline of foundation) to many constructions and concepts in terms of Lorentzian geometry.

Let us fix once for ever some base surfaces that will support several geometric structures:

$$
(\hat{S}, V)
$$

is a compact closed oriented surface of genus $g \geq 0$, with a set of $r \geq 0$ marked points $V=\left\{p_{1}, \cdots, p_{r}\right\}$

$$
S=\hat{S} \backslash V .
$$

$\bar{\Sigma}$ is obtained by removing from $\hat{S}$ a small open disk around each point $p_{j}$. Hence $\bar{\Sigma}$ is compact with $r$ boundary components $C_{1}, \cdots, C_{r}$. We denote by $\Sigma$ the interior of $\bar{\Sigma}$. We fix also a continuous map

$$
\bar{\phi}: \bar{\Sigma} \rightarrow \hat{S}
$$

such that for every $j, \bar{\phi}\left(C_{j}\right)=p_{j}$, and the restriction $\phi: \Sigma \rightarrow S$ is an oriented diffeomorphism that is the identity outside a regular neighbourhood of the boundary of $\bar{\Sigma}$. In this way, we will often tacitly confuse $S$ and $\Sigma$. We will also assume that $S$ is not elementary, that is its fundamental group is non-Abelian, equivalently $2-2 g-r<0$. Such an $S$ is said to be of finite type.

\subsection{The Teichmüller space $\widetilde{\mathcal{T}}(S)$. We denote by}

$$
\widetilde{\mathcal{H}}(S)
$$

the space of non-necessarily complete hyperbolic structures $F$ on $S$ such that its completion $F^{\mathcal{C}}$ is a complete hyperbolic surface with geodesic boundary. Note that we do not require that the boundary components of $F^{\mathcal{C}}$ are closed geodesics. Denote by Diff ${ }^{0}$ the group of diffeomorphisms of $S$ homotopic to the identity. Set

$$
\widetilde{\mathcal{T}}(S)=\widetilde{\mathcal{H}}(S) / \operatorname{Diff}^{0}
$$

in other words, two hyperbolic structures in $\widetilde{\mathcal{H}}(S)$ are identified up to isometries homotopic to the identity. This is the "full" Teichmüller space we will deal with.

3.2. The convex-core map. Let us point out some distinguished subspaces of $\widetilde{\mathcal{T}}(S)$.

$$
\mathcal{H}(S) \subset \widetilde{\mathcal{H}}(S)
$$

denotes the space of complete hyperbolic structures on $S$ (i.e. $F=F^{\mathcal{C}}$ ). Hence every $F \in \mathcal{H}(S)$ can be realized as the quotient $\mathbb{H}^{2} / \Gamma$ by a discrete, torsion free subgroup $\Gamma \subset \operatorname{Isom}^{+}\left(\mathbb{H}^{2}\right) \cong P S L(2, \mathbb{R})$, isomorphic to $\pi_{1}(S)$. The corresponding quotient space

$$
\mathcal{T}(S) \subset \widetilde{\mathcal{T}}(S)
$$

can be identified with the space of conjugacy classes of such subgroups of $P S L(2, \mathbb{R})$.

$$
\mathfrak{C}(S) \subset \widetilde{\mathcal{H}}(S)
$$


denotes the set of $F$ of finite area and such that all boundary components of $F^{\mathcal{C}}$ are closed geodesics.

$$
\mathcal{T}_{\mathfrak{c}}(S) \subset \widetilde{\mathcal{T}}(S)
$$

is the corresponding quotient space.

Clearly if $S$ is compact $(V=\emptyset)$

$$
\mathcal{T}_{g}:=\mathcal{T}_{\mathfrak{c}}(S)=\mathcal{T}(S)=\widetilde{\mathcal{T}}(S)
$$

is the classical Teichmüller space.

In general, set

$$
\mathcal{T}_{g, r}:=\mathcal{T}(S) \cap \mathcal{T}_{\mathfrak{c}}(S) .
$$

Via Uniformization Theorem, $\mathcal{T}_{g, r}$ is isomorphic to the Teichmüller space of conformal structures on $\hat{S}$ (i.e. on $S$ that extend to $\hat{S}$ ) $\bmod \operatorname{Diff}^{0}(\hat{S}$, rel $V)$. $\mathcal{T}(S)$ is isomorphic to the Teichmüller space of arbitrary conformal structures on $S$.

Proposition 3.1. There is an natural isomorphism

$$
\mathcal{K}: \mathcal{T}(S) \rightarrow \mathcal{T}_{\mathfrak{c}}(S)
$$

Basically $\mathcal{K}[F]$ coincides with $[\mathcal{K}(F)]$, where $\mathcal{K}(F)$ denotes the interior of the convex core $\overline{\mathcal{K}}(F)$ of $F$. Note that $\mathcal{K}(F)^{\mathcal{C}}=\overline{\mathcal{K}}(F)$. This is a bijection because the convex core determines the whole complete surface.

Proposition 3.2. There is a natural projection

$$
\beta: \widetilde{\mathcal{T}}(S) \rightarrow \mathcal{T}(S)
$$

such that $\beta_{\mid \mathcal{T}(S)}=\mathrm{Id}$.

In fact the holonomy of any $[F] \in \widetilde{\mathcal{T}}(S)$ is the conjugacy class of a faithful representation of $\pi_{1}(S)$ onto a discrete, torsion free subgroup $\Gamma$ of $P S L(2, \mathbb{R})$, hence $\beta([F])=[\hat{F}]$, $\hat{F}=\mathbb{H}^{2} / \Gamma$. Finally we can lift the map of Proposition 3.1 to define the convex-core map

$$
\mathcal{K}: \tilde{\mathcal{T}}(S) \rightarrow \mathcal{T}_{\mathfrak{c}}(S), \quad \mathcal{K}([F])=\mathcal{K}([\hat{F}]) .
$$

In fact we can realize the representatives of the involved classes in such a way that

$$
\overline{\mathcal{K}}(\hat{F}) \subset F^{\mathcal{C}} \subset \hat{F}
$$

for $F^{\mathcal{C}}$ is a closed convex set in $\hat{F}$ homotopically equivalent to $S$, and $\overline{\mathcal{K}}(\hat{F})$ is the minimal one with these properties. In what follows we will often made the abuse of confusing the classes with their representatives.

Partition by types.

Proposition 3.3. For every complete surface $F \in \mathcal{T}(S)$ there is a partition

$$
V=V_{\mathcal{P}} \cup V_{\mathcal{H}}
$$

such that $p$ belongs to $V_{\mathcal{P}}$ iff the following equivalent properties are satisfied:

(1) $F$ is of finite area at $p$ (that is $F$ has a cusp at $p$ );

(2) the holonomy of a circle in $S$ surrounding $p$ is of parabolic type;

$p$ belongs to $V_{\mathcal{H}}$ iff the following equivalent properties are satisfied: 
(i) p corresponds to a boundary component of the convex core $\overline{\mathcal{K}}(F)$;

(ii) the holonomy of a circle in $S$ surrounding $p$ is of hyperbolic type.

The partition $V=V_{\mathcal{P}} \cup V_{\mathcal{H}}$, so that $r=r_{\mathcal{P}}+r_{\mathcal{H}}$, is said the type $\theta(F)$ of $F$. More generally, for every $F \in \widetilde{\mathcal{T}}(S)$, set $\theta(F)=\theta(\hat{F})$. Any fixed type $\theta$ determines the subspace $\widetilde{\mathcal{T}}^{\theta}(S)$ of hyperbolic structures that share that type; varying $\theta$ we get the partition by types of $\widetilde{\mathcal{T}}(S)$.

\section{The fibers of the convex-core map.}

We want to describe the fibers of the convex-core map

$$
\mathcal{K}: \tilde{\mathcal{T}}(S) \rightarrow \mathcal{T}_{\mathfrak{c}}(S)
$$

Let $h \in \mathrm{Isom}^{+}\left(\mathbb{H}^{2}\right)$ be of hyperbolic type. Denote by $\gamma=\gamma_{h}$ its invariant geodesic. Let $P$ be the closed hyperbolic half-plane determined by $\gamma$ such that the orientation of $\gamma$ as boundary of $P$ is opposite to the sense of the translation $h_{\mid \gamma}$.

Definition 3.4. An $h$-crown is of the form

$$
\mathcal{E}=H / h
$$

where $H$ is the convex hull in $P$ of a $h$-invariant closed subset, say $\mathcal{E}_{\infty} \subset S_{\infty}^{1}$, contained in the frontier at infinity of $P$.

An $h$-crown $\mathcal{E}$ is complete and has geodesic boundary made by the union of the closed geodesic $\gamma / h$ and complete open geodesics. $\mathcal{E} \backslash \partial \mathcal{E}$ is homeomorphic to $S^{1} \times(0,+\infty)$. Now, let $F \in \widetilde{\mathcal{T}}^{\theta}(S)$ and $\overline{\mathcal{K}}(\hat{F}) \subset F^{\mathcal{C}} \subset \hat{F}$ be as above. Then $F^{\mathcal{C}}$ is obtained by gluing a (possibly empty) crown at each boundary component $C$ of $\overline{\mathcal{K}}(\hat{F})$, associated to some point $p \in V_{\mathcal{H}}$. This is possible iff, for every $C$ we take an $h$-crown $\mathcal{E}$ such that $h$ is in the same conjugacy class of the $\hat{F}$-holonomy of the loop $C$, endowed with the boundary orientation of $\overline{\mathcal{K}}(\hat{F})$ (in other words, length $(\gamma / h)=l(C)$ and both orientations of $\overline{\mathcal{K}}(\hat{F})$ and $\mathcal{E}$ are induced by the one of $\hat{F}$ ).

Lemma 3.5. $F$ is of finite area iff all crowns are. A crown $\mathcal{E}$ is of finite area iff one of the following equivalent conditions are satisfied:

(1) $\mathcal{E}_{\infty} / h$ is a finite set.

(2) $\mathcal{E}$ has finitely many boundary components. For every boundary component $l$, the distance between each end of $l$ and $\partial \mathcal{E} \backslash l$ is 0 .

Every h-crown $\mathcal{E}$ (every $F \in \widetilde{\mathcal{T}}(S)$ ) is the union of exhaustive sequences of increasing sub-crowns $\mathcal{E}_{n} \subset \mathcal{E}$ (sub-surfaces $F_{n} \subset F$ ) of finite area such that $\mathcal{E}_{n, \infty} \subset \mathcal{E}_{\infty}$.

In fact if $\mathcal{E}_{\infty}$ is finite, then the area of $\mathcal{E}$ can be bounded by the sum of the area of a finite set of ideal triangles. If $\mathcal{E}_{\infty}$ is not a finite set, then $\mathcal{E}$ contains an infinite family of disjoint ideal triangles.

Finally, for every $F \in \mathcal{T}_{\mathfrak{c}}(S)$, the fiber $\mathcal{K}^{-1}(F)$ can be identified with the set of all possible patterns of $r_{\mathcal{H}}$ gluable crowns.

Parameters for $\mathcal{T}_{\mathfrak{c}}(S)$. The fibers of the convex-core map are in any sense "infinite dimensional". On the other hand, the base space $\mathcal{T}_{\mathfrak{c}}(S)$ is tame and admits nice parameter spaces, that we are going to recall. 
Length/twist parameters. This is based on a fixed pant decomposition $\mathcal{D}$ of $\bar{\Sigma}$. $\mathcal{D}$ contains $2 g+r-2$ pants obtained by cut/opening $\bar{\Sigma}$ at $3 g-3+r$ (ordered) disjoint essential simple closed curves $z_{1}, \cdots, z_{3 g-3+r}$ in $\Sigma$, not isotopic to any boundary component. Everyone of the $r$ boundary components $C_{1}, \cdots, C_{r}$ of $\bar{\Sigma}$ is in the boundary of some pant. For every boundary component of a pant $P_{k}$, corresponding to some $z_{j}$, we fix also the unique "essential" $\operatorname{arc} \rho$ in $P_{k}$ (shown in Fig. 1) that has the end-points on that component, and we select furthermore one among these end-points, say $e$.

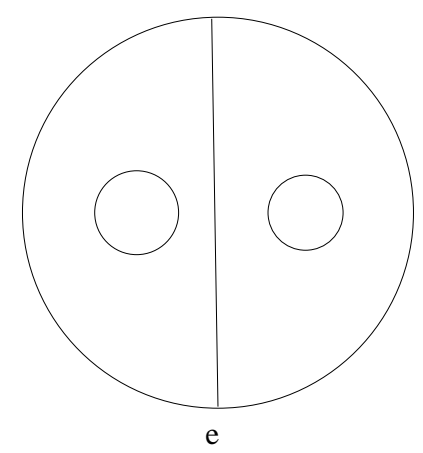

Figure 1. A pant and an $\operatorname{arc} \rho$.

Denote by

$$
\mathbb{R}_{+}=\{l \in \mathbb{R} ; l>0\}, \quad \overline{\mathbb{R}}_{+}=\{l \in \mathbb{R} ; l \geq 0\} .
$$

Consider first the simplest case of $S$ having $(g, r)=(0,3)$. In this case, set

$$
\mathcal{T}_{\mathfrak{c}}(S)=\mathcal{T}_{\mathfrak{c}}(0,3)
$$

We have just one pant. Let us vary the types. If $r_{\mathcal{H}}=3$, every hyperbolic structure is determined by the 3 lengths $\left(l_{1}, l_{2}, l_{3}\right)$ of the geodesic boundary components. If $r_{\mathcal{H}}=2$, by the corresponding 2 lengths, and it is natural to associate the value 0 to the boundary component that corresponds to the cusp, and so on. Eventually the octant

$$
\overline{\mathbb{R}}_{+}^{3}=\left\{\left(l_{1}, l_{2}, l_{3}\right) ; l_{j} \geq 0\right\}
$$

is a natural parameter space for the whole $\mathcal{T}_{\mathfrak{c}}(0,3)$. The canonical stratification by open cells of this closed octant corresponds to the partition by types.

In the general case, let $F \in \mathcal{T}_{\mathfrak{c}}(S)$; then every pant of the topological decomposition $\mathcal{D}$ is associated to a suitable hyperbolic pant $P_{i}=P_{i}(F)$ belonging to $\mathcal{T}_{\mathfrak{c}}(0,3)$. Pant geodesic boundary components corresponding to some curve $z_{j}$ have the same length, so that $F^{\mathcal{C}}$ is obtained by isometrically gluing the hyperbolic pants at the curves $z_{j}$. Summing up, $F$ is of the form

$$
\begin{gathered}
F=F(l, t) \\
(l, t)=\left(l_{C_{1}}, \cdots, l_{C_{r}}, l_{z_{1}}, \cdots l_{z_{3 g-3+r}}, t_{z_{1}}, \cdots, t_{z_{3 g-3+r}}\right)
\end{gathered}
$$

where $l_{C_{i}}\left(l_{z_{j}}\right)$ is the length of the geodesic boundary component (the simple closed geodesic) of $F^{\mathcal{C}}$ corresponding to $C_{i}\left(z_{j}\right)$. The twist parameter $t_{z_{j}} \in \mathbb{R}$ specifies the isometric gluing at $z_{j}$ as follows. For every hyperbolic pant, an arc $\rho$ is uniquely realized by a geodesic arc orthogonal to the boundary. Then $F(l, 0)$ is the unique hyperbolic structure such that the selected end-points $e$ of such geometric $\rho$-arcs match by gluing. 
A generic $F(l, t)$ is obtained from $F(l, 0)$ by modifying the gluing as follows: if $t_{z_{j}}>0$, the two sides at any geodesic line $\widetilde{z}_{j}$ in $\mathbb{H}^{2}$ over the closed geodesic $z_{j}$ of $F(l, 0)$ translate by $t_{z_{j}}$ along $\widetilde{z}_{j}$ on the left to each other. If $t_{z_{j}}<0$, they translate on the right by $\left|t_{z_{j}}\right|$ ("left" and "right" are well defined and only depend on the orientation of $S$ ).

We eventually realize in this way that

$$
\overline{\mathbb{R}}_{+}^{r} \times \mathbb{R}_{+}^{3 g-3+r} \times \mathbb{R}^{3 g-3+r}
$$

is a parameter space (depending on the choice of $\mathcal{D}$ ) for the whole $\mathcal{T}_{\mathfrak{c}}(S)$. The product by $\mathbb{R}_{+}^{3 g-3+r} \times \mathbb{R}^{3 g-3+r}$ of the natural stratification by open cells of $\overline{\mathbb{R}}_{+}^{r}$, corresponds to the partition by types. Every cell has dimension

$$
6 g-6+2 r+r_{\mathcal{H}}
$$

according to the type. The top-dimensional cell $\left(r_{\mathcal{H}}=r\right)$ corresponds to the hyperbolic surfaces $F$ without cusps. $\mathcal{T}_{g, r}$ is the lowest dimensional one. Cells that share the same $r_{\mathcal{H}}$ are isomorphic as well as the corresponding $\mathcal{T}_{\mathcal{C}}^{\theta}(S)$. By varying $\mathcal{D}$ we actually get an atlas for $\mathcal{T}_{\mathfrak{c}}(S)$ that gives it a real analytic manifold with corner structure.

Marked length spectrum. Length and twist parameters are of somewhat different nature; in fact we can deal with length parameters only. For every $j$, consider: the "double pant" obtained by gluing the two pants of $\mathcal{D}$ at $z_{j}$; the simple closed curve $z_{j}^{\prime}$ obtained by gluing the respective two $\rho$ arcs, and $z_{j}^{\prime \prime}$ the curve obtained from $z_{j}^{\prime}$ via a Dehn twist along $z_{j}$. Thus we have further $6 g-6+2 r$ simple closed curves on $S$, and for every $F$ we take the length of the corresponding simple closed geodesics. In this way we get an embedding

$$
\mathcal{T}_{\mathfrak{c}}(S) \subset \overline{\mathbb{R}}_{+}^{r} \times \mathbb{R}_{+}^{9 g-9+3 r}
$$

This is the projection onto this finite set of factors of the marked length spectrum injection

$$
\mathrm{L}: \mathcal{T}_{\mathfrak{c}}(S) \rightarrow \overline{\mathbb{R}}_{+}^{r} \times \mathbb{R}_{+}^{\mathfrak{S}^{\prime}}
$$

where $\mathfrak{S}^{\prime}$ denotes the set of isotopy classes of essential simple closed curves in $S$, not isotopic to any boundary component.

For more details about the length/twist parameters and the length spectrum see for instance [25, 12].

Shear parameters. This is based on a fixed topological ideal triangulation $T$ of $(\hat{S}, V)$, and works only if $V \neq \emptyset$. By definition $T$ is a (possibly singular - multi and self adjacency of triangles are allowed) triangulation of $\hat{S}$ such that $V$ coincides with the set of vertices of $T$. There are $6 g-6+3 r$ edges $E_{1}, \cdots, E_{6 g-6+3 r}$. The idea is to consider every triangle of $T$ as a hyperbolic ideal triangle and realize hyperbolic structures $F$ on $S$ by isometrically gluing them at the geodesic edges, according to the pattern of edge-identifications given by $T$. By the way, $T$ will be converted in a geometric ideal triangulation $T_{F}$ of $F$. Let us decorate every edge $E$ of $T$ by a real number $s_{E}$ and get

$$
s=\left(s\left(E_{1}\right), \cdots, s\left(E_{6 g-6+3 r}\right)\right) \in \mathbb{R}^{6 g-6+3 r} .
$$

These shear parameters encode the isometric gluing at each $E_{j}$, and are of the same nature of the above twist parameters. Every edge of an ideal triangle has a distinguished point, say $e$, that is the intersection of the edge with the unique geodesic line emanating from the opposite ideal vertex and which is orthogonal to it. Then set $F=F(0)$ to 
be the unique hyperbolic structure such that the distinguished points match by gluing. A generic $F=F(s)$ is obtained from $F(0)$ by modifying the gluing according to the left/right moving rule as before. It turns out that all so obtained hyperbolic structures $F$ belong to $\mathcal{T}_{\mathfrak{c}}(S)$, and all elements of $\mathcal{T}_{\mathfrak{c}}(S)$ arise in this way. For every $s$ and every $p_{i} \in V$, set

$$
s\left(p_{i}\right)=\sum_{E_{j} \in \operatorname{Star}\left(p_{i}\right)} s\left(E_{j}\right) .
$$

We realize that

$$
l_{C_{i}}(F(s))=\left|s\left(p_{i}\right)\right|
$$

so that, in particular, $p_{i} \in V_{\mathcal{P}}$ iff $s\left(p_{i}\right)=0$ and this determines the type $\theta=\theta(F(s))$. This also shows that the map

$$
\mathcal{S}: \mathbb{R}^{6 g-6+3 r} \rightarrow \mathcal{T}_{\mathfrak{c}}(S), \quad F=F(s)
$$

is not injective. For every $p_{i} \in V_{\mathcal{H}}$, define the sign $\epsilon_{s}\left(p_{i}\right)$ by

$$
\left|s\left(p_{i}\right)\right|=\epsilon_{s}\left(p_{i}\right) s\left(p_{i}\right) .
$$

Then, the generic fiber $\mathcal{S}^{-1}(F)$ consists of $2^{r_{\mathcal{H}}}$ points, that is $\mathcal{S}$ realizes all the possible signature $V_{\mathcal{H}} \rightarrow\{ \pm 1\}$. For the geometric meaning of these signs, see below. For more details about shear parameters see for instance [14](4).

The enhanced $\mathcal{T}_{\mathfrak{c}}(S)^{\#}$. Let us reflect a length/twist parameter space

$$
\overline{\mathbb{R}}_{+}^{r} \times \mathbb{R}_{+}^{3 g-3+r} \times \mathbb{R}^{3 g-3+r}
$$

of $\mathcal{T}_{\mathfrak{c}}(S)$ along its boundary components to get

$$
\mathbb{R}^{r} \times \mathbb{R}_{+}^{3 g-3+r} \times \mathbb{R}^{3 g-3+r} .
$$

This can be considered as a parameter space of the enhanced Teichmüller space $\mathcal{T}_{\mathfrak{c}}(S)^{\#}$, obtained by decorating each $F$ with a signature

$$
\epsilon: V_{\mathcal{H}} \rightarrow\{ \pm 1\} \text {. }
$$

Moreover, we stipulate that the sign $\epsilon_{i}$ associated to $i$ has the meaning of selecting an orientation of the corresponding $C_{i}$, by the rule: $\epsilon_{i}=+1$ iff $C_{i}$ is equipped with the boundary orientation.

To make the notation simpler, it is convenient to extend the signature $\epsilon$ to the whole of $V$ by stating that $\epsilon_{i}=1$ on $V_{\mathcal{P}}$. In this way an enhanced surface can be written as $\left(F, \epsilon_{1}, \ldots, \epsilon_{r}\right)$ with $\epsilon_{i} \in\{ \pm 1\}$ and $\epsilon_{i}=1$ for $i$ corresponding to a cusp of $F$.

In the same way one can show that the shearing parameters are global coordinates on $\mathcal{T}_{\mathfrak{c}}(S)^{\#}$, namely the map

$$
\mathcal{S}^{\#}: \mathbb{R}^{6 g-6+3 r} \rightarrow \mathcal{T}_{C}^{\#}(S)
$$

defined by $\mathcal{S}^{\#}(s)=\left(F(s), \operatorname{sign}\left(s\left(p_{1}\right)\right), \ldots, \operatorname{sign}\left(s\left(p_{n}\right)\right)\right.$ ), is a homeomorphism (see [26] for details).

There is a natural forgetting projection

$$
\phi^{\#}: \mathcal{T}_{\mathfrak{c}}(S)^{\#} \rightarrow \mathcal{T}_{\mathfrak{c}}(S) .
$$

We can also define in a coherent way the enhanced length spectrum

$$
\mathrm{L}^{\#}: \mathcal{T}_{\mathfrak{c}}(S)^{\#} \rightarrow \mathbb{R}^{r} \times \mathbb{R}_{+}^{\mathfrak{S}^{\prime}}
$$


by setting

$$
l_{C_{i}}^{\#}(F, \epsilon)=\epsilon_{i} l_{C_{i}}(F)
$$

on the peripheral loops, and $l_{\gamma}^{\#}(F, \epsilon)=l_{\gamma}(F)$ elsewhere. This is an injection of $\mathcal{T}_{\mathfrak{c}}(S)^{\#}$, and already the projection onto the usual finite set of factors as above is an embedding.

Remark 3.6. For each $C_{i}$, the enhanced length is a continuous function of $\mathcal{T}_{\mathfrak{c}}^{\#}(S)$. On the other hand notice that $\epsilon_{i}$ coincides with the sign of $l_{C_{i}}^{\#}$, with the rule that the sign of 0 is 1 .

\subsection{The space of measured geodesic laminations.}

Definition 3.7. A simple (complete) geodesic in $F \in \tilde{\mathcal{T}}(S)$ is a geodesic which admits an arc length parametrization defined on the whole real line $\mathbb{R}$ that either is injective (and we call its image a geodesic line of $F$ ), or its image is a simple closed geodesic. A geodesic lamination $\mathcal{L}$ on $F$ consists of:

(1) A closed subset $L$ of $F$ (the support);

(2) A partition of $L$ by simple geodesics (the leaves).

The leaves together with the connected components of $F \backslash L$ make a stratification of $S$.

Definition 3.8. Given a geodesic lamination $\mathcal{L}$ on $F \in \tilde{\mathcal{T}}(S)$, a rectifiable arc $k$ in $F$ is transverse to the lamination if for every point $p \in k$ there exists a neighbourhood $k^{\prime}$ of $p$ in $k$ that intersects each leaf in at most a point and each 2-stratum in a connected set. A transverse measure $\mu$ on $\mathcal{L}$ is the assignment of a positive measure $\mu_{k}$ on each rectifiable arc $k$ transverse to $\mathcal{L}$ (this means that $\mu_{k}$ assigns a non-negative mass $\mu_{k}(A)$ to every Borel subset of the arc, in a countably additive way) in such a way that:

(1) The support of $\mu_{k}$ is $k \cap L$;

(2) If $k^{\prime} \subset k$, then $\mu_{k^{\prime}}=\left.\mu_{k}\right|_{k^{\prime}}$;

(3) If $k$ and $k^{\prime}$ are homotopic through a family of arcs transverse to $\mathcal{L}$, then the homotopy sends the measure $\mu_{k}$ to $\mu_{k^{\prime}}$;

Notice that we allow an arc $k$ hitting the boundary of $F^{\mathcal{C}}$ to have infinite mass, that is $\mu_{k}(k)=+\infty$.

Definition 3.9. A measured geodesic lamination on $F$ is a pair $\lambda=(\mathcal{L}, \mu)$, where $\mathcal{L}$ is a geodesic lamination and $\mu$ is a transverse measure on $\mathcal{L}$. For every $F \in \widetilde{\mathcal{T}}(S)$, denote by $\mathcal{M L}(F)$ the set of measured geodesic laminations on $F$. Finally, let us define $\mathcal{M L}(S)$ to be the set of couples $(F, \lambda)$, such that $F \in \widetilde{\mathcal{T}}(S)$, and $\lambda \in \mathcal{M L}(F)$. We have the natural projection

$$
\mathfrak{p}: \mathcal{M L}(S) \rightarrow \widetilde{\mathcal{T}}(S)
$$

Definition 3.10. Given $(F, \lambda) \in \mathcal{M L}(S)$, the simplicial part $\mathcal{L}_{S}$ of $\mathcal{L}$ consists of the union of the isolated leaves of $\mathcal{L}$. Hence $\mathcal{L}_{S}$ does not depend on the measure $\mu$. A leaf, $l$, is called weighted if there exists a transverse arc $k$ such that $k \cap l$ is an atom of $\mu_{k}$. The weighted part of $\lambda$ is the union of all weighted leaves. It depends on the measure and it is denoted by $\mathcal{L}_{W}=\mathcal{L}_{W}(\mu)$. 
Remark 3.11. The word "simplicial" mostly refers to the "dual" geometry of the initial singularity of the spacetimes that we will associate to every $(F, \lambda)$, see Section 4 .

By property (3) of the definition of transverse measure, if $l$ is weighted then for every transverse arc $k$ the intersection of $k$ with $l$ consists of atoms of $\mu_{k}$ whose masses are equal to a positive number $A$ independent of $k$. We call this number the weight of $l$. Since every compact set $K \subset F$ intersects finitely many weighted leaves with weight bigger than $1 / n$, it follows that $\mathcal{L}_{W}$ is a countable set. As $L$ is the support of $\mu$, then we have the inclusion $\mathcal{L}_{S} \subset \mathcal{L}_{W}(\mu)$.

Remark 3.12. There is a slightly different but equivalent definition of $\mathcal{M L}(S)$ that runs as follows. We can consider measured geodesic laminations $\lambda=(\mathcal{L}, \mu)$ of $F^{\mathcal{C}}$ requiring furthermore that:

(1) The boundary components of $F^{\mathcal{C}}$ are leaves of $\mathcal{L}$;

(2) Every arc $k$ hitting the boundary of $F^{\mathcal{C}}$ necessarily has infinite mass $\left(\mu_{k}(k)=+\infty\right)$.

If a boundary component of $F^{\mathcal{C}}$ is isolated in $\mathcal{L}$ we stipulate that it has weight $+\infty$. Notice that while a geodesic lamination on $F^{\mathcal{C}}$ can be regarded also as a particular lamination on the associated complete surface $\hat{F}$, condition (2) ensures that such a measured lamination cannot be extended beyond $F^{\mathcal{C}}$. On the other hand, a lamination on $F$ in not in general a lamination on $\hat{F}$.

Given any $\lambda$ of $F$ we get a corresponding $\hat{\lambda}$ of $F^{\mathcal{C}}$ by adding the (possibly $+\infty$-weighted) boundary components to the lamination and keeping the same measure. Given $\hat{\lambda}$ in $F^{\mathcal{C}}$ we get $\lambda$ in $F$ by just forgetting the boundary leaves. In particular the empty lamination on $F$ corresponds to the lamination on $F^{\mathcal{C}}$ reduced to its boundary components. Clearly this establishes a canonical bijection, hence an equivalent definition of $\mathcal{M L}(S)$. This second definition would sound at present somewhat unmotivated, so in this section we prefer to deal with $F$ instead of $F^{\mathcal{C}}$. However, we will see in Section 4 that it is the suitable one when dealing with the Lorentzian "materializations" of $\mathcal{M L}(S)$.

Marked measure spectrum. Similarly to the above length spectrum L, for every $F \in \widetilde{\mathcal{T}}(S)$, it is defined the marked measure spectrum

$$
\mathrm{I}: \mathcal{M L}(F) \rightarrow \overline{\mathbb{R}}_{+}^{r} \times \overline{\mathbb{R}}_{+}^{\mathfrak{S}^{\prime}}
$$

where for every $\lambda \in \mathcal{M L}(F)$ and for every isotopy class $\gamma$ of essential simple closed curves on $S, \mathrm{I}_{\gamma}(\lambda)$ is the minimum of the total variation $\mu(c)$ of the " $\lambda$-transverse component" of $c, c$ varying among the representatives of $s$. The first $r$ factors correspond as usual to the curves parallel to the boundary components.

Ray structure. Every $\lambda=(\mathcal{L}, \mu) \in \mathcal{M L}(F)$ determines the ray

$$
R_{\lambda}=\{t \lambda=(\mathcal{L}, t \mu) ; t \in[0,+\infty)\} \subset \mathcal{M L}(F)
$$

where we stipulate that for $t=0$ we take the empty lamination of $F$. If $\mathrm{I}_{\lambda} \neq 0$, then $\mathrm{I}\left(R_{\lambda}\right)=R_{\mathrm{I}_{\lambda}}$, that is the corresponding ray in $\overline{\mathbb{R}}_{+}^{\mathfrak{S}}$. 


\subsection{The sub-space $\mathcal{M} \mathcal{L}_{\mathfrak{c}}(S)$.}

$$
\begin{gathered}
\mathcal{M} \mathcal{L}_{\mathfrak{c}}(S)=\left\{(F, \lambda) \in \mathcal{M L}(S) ; F \in \mathcal{T}_{\mathfrak{c}}(S)\right\} \\
\mathfrak{p}_{\mathfrak{c}}: \mathcal{M} \mathcal{L}_{\mathfrak{c}}(S) \rightarrow \mathcal{T}_{\mathfrak{c}}(S)
\end{gathered}
$$

being the natural restriction of $\mathfrak{p}$ with fibers $\mathcal{M} \mathcal{L}_{\mathfrak{c}}(F)$.

For any $F \in \mathcal{T}_{\mathfrak{c}}(S)$ denotes by $\mathcal{M L}_{\mathfrak{c}}(F)^{0}$ the set of laminations on $F$ that do not enter any cusp (namely the closure in $F^{\mathcal{C}}$ of the lamination support is compact). For a fixed type $\theta$ we denotes by

$$
\mathcal{M L}_{\mathfrak{c}}(S)^{\theta}=\left\{(F, \lambda) \mid F \in \mathcal{T}_{\mathfrak{c}}^{\theta}(S), \lambda \in \mathcal{M} \mathcal{L}_{\mathfrak{c}}(S)^{0}\right\}
$$

and we still denote by $\mathfrak{p}_{\mathfrak{c}}$ the restriction of the projection on every $\mathcal{M L}_{\mathfrak{c}}(S)^{\theta}$.

The spectrum I and the ray structure naturally restrict themselves. In particular, if $\lambda \in \mathcal{M L}_{\mathfrak{c}}(F)^{0}$, and $s$ surrounds a cusp of $F$, then $\mathrm{I}_{\lambda}(s)=0$. On the other hand, if $s$ is parallel to a boundary component of $F^{\mathcal{C}}$, then $\mathrm{I}_{\lambda}(s)=0$ iff the closure in $F^{\mathcal{C}}$ of the lamination support $L$ does not intersect that boundary component.

The following Proposition summarizes some basic properties of the fibers of $\mathfrak{p}_{\mathfrak{c}}$.

Proposition 3.13. Let $\lambda \in \mathcal{M L}_{\mathfrak{c}}(F)$. Then:

(1) $F \backslash L$ has a finite number of connected components, and each component belongs to some $\widetilde{\mathcal{T}}\left(S^{\prime}\right)$, providing that we drop out the requirement that $S^{\prime}$ is non-elementary.

(2) $\lambda$ is disjoint union of a finite set of minimal [with respect to the inclusion] measured sublaminations [recall that a lamination $\mathcal{L}$ is minimal iff every half-leaf is dense in $\mathcal{L}$ ]. Every minimal sublamination either is compact or consists of a geodesic line such that each sub half-line either enters a cusp or spirals towards a boundary component of $F^{\mathcal{C}}$. (3) $\mathcal{L}_{W}=\mathcal{L}_{S}$

(4) Either any cusp or any boundary component has a neighbourhood $U$ such that $\mathcal{L} \cap U=\mathcal{L}_{S} \cap U$.

(5) For every arc $c$ in $F$ transverse to $\lambda, c \cap L$ is union of isolated points and of a finite union of Cantor sets.

For a proof when $F \in \mathcal{T}_{g, r}$ we refer for instance to the body and the references of [14](1). The details for the extension to the whole of $\mathcal{M} \mathcal{L}_{\mathfrak{c}}(S)$ are given for instance in [19].

Remark 3.14. If the lamination $\hat{\lambda}$ of $F^{\mathcal{C}}$ corresponds to $\lambda$ of $F$ as in Remark 3.12, then a leaf spiraling towards a boundary component of $F^{\mathcal{C}}$ as in (2) is no longer a minimal sublamination of $\hat{\lambda}$.

Example 3.15. We refer to the above length/twist or shear parameters for $\mathcal{T}_{\mathfrak{c}}(S)$.

(a) Let $F=F(l, t)$. The union of simple closed geodesics of $F$ corresponding the the curves $z_{j}$ is a geodesic lamination $\mathcal{L}=\mathcal{L}_{S}$ of $F$. By giving each $z_{j}$ an arbitrary real weight $w_{j}>0$, we get $\lambda(w) \in \mathcal{M L}_{\mathfrak{c}}(F(l, t))^{0}$.

(b) Let $F=F(s)$. The 1-skeleton of the geometric ideal triangulation $T_{F}$ (which is made by geodesic lines) makes a geodesic lamination of $F$. Every geodesic line is a 
minimal sublamination. By giving each geodesic lines an arbitrary weight $w_{j}>0$, we get $\lambda(w) \in \mathcal{M L}_{\mathfrak{c}}(F(s))$. For such a $\lambda=\lambda(w)$

$$
\mathrm{I}_{C_{i}}(\lambda)=\sum_{E_{j} \in \operatorname{Star}\left(p_{i}\right)} w\left(E_{j}\right) .
$$

Lamination signatures. Let $\lambda \in \mathcal{M L}_{\mathfrak{c}}(F)$. Leaves of $\lambda$ can spiral around a boundary component $C_{i}$ in two different ways. On the other hand two leaves that spiral around $C_{i}$ must spiral in the same way (otherwise they would meet each other).

This determines a signature

$$
\sigma(\lambda): V_{\mathcal{H}} \rightarrow\{ \pm 1\}
$$

such that $\sigma_{i}(\lambda)=-1$ if and only if there are leaves of $\lambda$ spiraling around the corresponding geodesic boundary $C_{i}$ with a negative sense with respect to the boundary orientation. In other words, $\sigma_{i}(\lambda)$ is possibly equal to -1 only if $p_{i} \in V_{\mathcal{H}}$ and $\mathrm{I}_{C_{i}}(\lambda) \neq 0$, $\sigma_{i}(\lambda)=1$ otherwise. The signature depends indeed only on the lamination $\mathcal{L}$, not on the measure.

Remark 3.16. If $\lambda=\lambda(w)$ as in Example 3.15(b), then $\sigma_{\lambda}$ recovers the signs $\epsilon_{s}\left(p_{i}\right)$ already defined at the end of Section 3.2 .

3.5. Enhanced bundle $\mathcal{M} \mathcal{L}_{\mathfrak{c}}(S)^{\#}$ and measure spectrum. Here we address the question to which extent the (restricted) marked measure spectrum determines $\mathcal{M} \mathcal{L}_{\mathfrak{c}}(S)$. For example, this is known to be the case if we restrict to $\mathcal{M} \mathcal{L}_{g, r}^{0}$ i.e. to laminations over $\mathcal{T}_{g, r}$ that do not enter the cusps (see for instance [14](1)). We want to extend this known result.

We have seen in Proposition 3.13 that a measured geodesic lamination $\lambda$ on $F \in \mathcal{T}_{\mathfrak{c}}(S)$ is the disjoint union of a compact part, say $\lambda_{c}$ (that is far away from the geodesic boundary of $F^{\mathcal{C}}$ and does not enter any cusps), with a part, say $\lambda_{b}$, made by a finite set of weighted geodesic lines $l_{1}, \ldots, l_{n}$ whose ends leave every compact subset of $F$. Notice that $\sigma(\lambda)=\sigma\left(\lambda_{b}\right)$.

Let us take such a geodesic line $l$ on $F \in \mathcal{T}_{\mathfrak{c}}(S)$. We can select a compact closed interval $J$ in $l$ such that both components of $l \backslash J$ definitely stay either within a small $\epsilon$-neigbourhood of some boundary component of $F^{\mathcal{C}}$, or within some cusp. $J$ can be completed to a simple arc $c$ in $\hat{S}$ with end-points in $V$, just by going straightly from each end-point of $J$ to the corresponding puncture. It is easy to see that the homotopy class with fixed end-points of the so obtained arc $c$ does not depend on the choice of $J$. For simplicity we refer to it as the "homotopy class" of $l$. We can also give the end-points of $c$ a sign \pm 1 in the very same way we have defined the signature of a lamination on $F$ (recall that the sign is always equal to 1 at cusps). We can prove

Lemma 3.17. Given any $F \in \mathcal{T}_{\mathfrak{c}}(S)$, every homotopy class $\alpha$ of simple arcs on $\hat{S}$ with end-points on $V$, and every signature of the end-points (compatible with the type of $F$ ) can be realized by a unique geodesic line l of $F$ whose ends leave every compact set of $F$. Moreover, the members of a finite family of such geodesic lines are pairwise disjoint iff the signs agree on every common end-point and there are disjoint representatives with end-points on $V$ of the respective homotopy classes. Analogously they do not intersect a compact lamination $\lambda_{c}$ iff so do suitable representatives. 
By using the lemma, we can prove (see [19])

Proposition 3.18. Let $\lambda \in \mathcal{M} \mathcal{L}_{\mathfrak{c}}(F)$. Then the support of $\lambda_{b}$ is determined by the homotopy classes of its geodesic lines $l_{i}$ and the signature of $\lambda$. More precisely, given any $\lambda_{c}$, every finite set of homotopy classes of simple weighted arcs on $\hat{S}$, with signed end-points in $V$ (providing the signature being compatible with the type of $F$ ), admitting representatives that are pairwise disjoint and do not intersect $\lambda_{c}$, is uniquely realized by a lamination $\lambda_{b}$ such that $\lambda=\lambda_{b} \cup \lambda_{c} \in \mathcal{M} \mathcal{L}_{\mathfrak{c}}(F)$.

Proposition 3.19. Let $F, F^{\prime} \in \mathcal{T}_{\mathfrak{c}}(S)$. Assume that $F$ is without cusps (that is $F$ belongs to the top dimensional cell of $\left.\mathcal{T}_{\mathfrak{c}}(S)\right)$. Then there is a natural map

$$
\iota: \mathcal{M L}_{\mathfrak{c}}(F) \rightarrow \mathcal{M} \mathcal{L}_{\mathfrak{c}}\left(F^{\prime}\right)
$$

such that for every (isotopy class of) simple closed curve $\gamma$ on $S$, we have

$$
\mathrm{I}_{\gamma}(\lambda)=\mathrm{I}_{\gamma}(\iota(\lambda)) .
$$

Proof : Assume first that $\lambda=\lambda_{c} \in \mathcal{M L}_{\mathfrak{c}}(F)$. Then there is a unique $\lambda^{\prime}=\lambda_{c}^{\prime} \in$ $\mathcal{M} \mathcal{L}_{\mathfrak{c}}\left(F^{\prime}\right)$ with the same spectrum. For we can embed $F^{\prime}$ in the double surfaces of $\left(F^{\prime}\right)^{\mathcal{C}}$, say $D F^{\prime}$ which is complete and of finite area. The measure spectrum of $\lambda_{c}$ induces a measure spectrum of a unique lamination $\lambda_{c}^{\prime \prime}$ on $D F^{\prime}$ (by applying the result on the spectrum in the special case recalled at the beginning of this Section). Finally we realize that the compact support of $\lambda_{c}^{\prime \prime}$ is contained in $F^{\prime}$ giving us the required $\lambda_{c}^{\prime}$. So the map $\iota$ can be defined for laminations with compact support.

Given a general lamination $\lambda=\lambda_{c} \cup \lambda_{b}$, we can define $\lambda_{c}^{\prime}$ as before, while $\lambda_{b}^{\prime}$ is the unique lamination of $F^{\prime}$ (accordingly with Proposition 3.18) that share with $\lambda_{b}$ the same homotopy classes, weights and signs at $V_{\mathcal{H}}(F) \cap V_{\mathcal{H}}\left(F^{\prime}\right)$. Notice that $\lambda_{b}^{\prime}$ is disjoint from $\lambda_{c}^{\prime}$ : in fact one can construct an isotopy of $S$ sending the supports of $\lambda_{b}$ and $\lambda_{c}$ to the supports of $\left.\lambda_{b}^{\prime}\right)$ and $\lambda_{c}^{\prime}$. Finally set $\iota(\lambda)=\iota\left(\lambda_{b}\right) \cup \iota\left(\lambda_{c}\right)$.

Corollary 3.20. If both $F$ and $F^{\prime}$ are without cusps, then the map $\iota$ is bijective. More generally, for every $\lambda^{\prime} \in \mathcal{M} \mathcal{L}_{\mathfrak{c}}\left(F^{\prime}\right), \iota^{-1}\left(\lambda^{\prime}\right)$ consists of $2^{k}$ points, where $k$ is the number of cusps of $F^{\prime}$ entered by $\lambda^{\prime}$.

In fact, for every $F \in \mathcal{T}_{\mathfrak{c}}(S)$ (not necessarily in the top dimensional cell), there is a natural action of $(\mathbb{Z} / 2 \mathbb{Z})^{r}$ on $\mathcal{M} \mathcal{L}_{\mathfrak{c}}(F)$ determined as follows. Let $\rho_{i}=(0, \ldots, 1, \ldots, 0)$, $i=1, \ldots r$, be the $i$ th element of the standard basis of $(\mathbb{Z} / 2 \mathbb{Z})^{r}$. Let $\lambda \in \mathcal{M} \mathcal{L}_{\mathfrak{c}}(F)$. First define the new signature $\rho_{i} \sigma(\lambda)$ by setting:

$\rho_{i} \sigma(\lambda)\left(p_{j}\right)=\sigma(\lambda)\left(p_{j}\right)$ if $i \neq j$;

$\left.\rho_{i} \sigma(\lambda)\left(p_{i}\right)=\sigma(\lambda)\right)\left(p_{i}\right)$ if either $p_{i} \in V_{\mathcal{P}}(F)$ or $p_{i} \in V_{\mathcal{H}}(F)$ and $\mathrm{I}_{C_{i}}(\lambda)=0$;

$\rho_{i} \sigma(\lambda)\left(p_{i}\right)=-\sigma(\lambda)\left(p_{i}\right)$, otherwise.

This naturally extends to every $\rho \in(\mathbb{Z} / 2 \mathbb{Z})^{r}$, giving the signature $\rho \sigma(\lambda)$. Finally set $\rho(\lambda)=\rho\left(\lambda_{b}\right) \cup \lambda_{c}$ where (accordingly again with Proposition 3.18) $\rho\left(\lambda_{b}\right)$ is the unique lamination that shares with $\lambda_{b}$ the homotopy classes and the weights, while its signature is $\rho \sigma(\lambda)$. Clearly the orbit of $\lambda$ consists of $2^{k}$ points, where $k$ is the number of $p_{i}$ in $V_{\mathcal{H}}(F)$ such that $\mathrm{I}_{C_{i}}(\lambda) \neq 0$. Finally $\iota^{-1}\left(\lambda^{\prime}\right)$ in Corollary 3.20 is just an orbit of such an action. We call the action on $\mathcal{M L}_{\mathfrak{c}}(F)$ of the generator $\rho_{i}$, the reflection along $C_{i}$ (even if it could be somewhat misleading, as in some case it is just the identity). 
If we restrict over the top-dimensional cell of $\mathcal{T}_{\mathfrak{c}}(S), \mathfrak{p}_{\mathfrak{c}}$ is a bundle and we can use the first statement of the Corollary in order to fix a trivialization. The same fact holds for every restriction $\mathfrak{p}_{\mathfrak{c}}: \mathcal{M L}_{\mathfrak{c}}(S)^{\theta} \rightarrow \mathcal{T}_{\mathfrak{c}}(S)^{\theta}$, type by type. On the other hand, because of the last statement of the Corollary, this is no longer true for the whole $\mathfrak{p}_{\mathfrak{c}}$. In order to overcome such phenomenon, one can introduce the notion of enhanced lamination. An enhanced lamination on $F \in \mathcal{T}_{\mathfrak{c}}(S)$, is a couple $(\lambda, \eta)$ where $\lambda \in \mathcal{M} \mathcal{L}_{\mathfrak{c}}(F)$, and $\eta: V \rightarrow\{ \pm\}$ is a relaxed signature such that:

$\eta_{i}=\sigma_{i}(\lambda)$ if either $p_{i} \in V_{\mathcal{H}}(F)$ or $p_{i} \in V_{\mathcal{P}}(F)$ and $\mathrm{I}_{C_{i}}(\lambda)=0$;

$\eta_{i}$ is arbitrary otherwise.

Notice that there are exactly $2^{k}(\lambda, \eta)$ enhancing a given $\lambda \in \mathcal{M L}_{\mathfrak{c}}(F)$, where $k$ is the number of cusps entered by $\lambda$. Clearly the above action of $(\mathbb{Z} / 2 \mathbb{Z})^{r}$ extends on enhanced laminations: $\rho(\lambda, \eta)=(\rho(\lambda), \rho(\eta))$, where $\rho(\eta)$ is uniquely determined by the above requirements and by the fact that $\rho \sigma(\lambda)$ possibly modifies $\sigma(\lambda)$ only on $V_{\mathcal{H}}$. In particular this holds for the generating reflections $\rho_{i}$.

We denote by $\mathcal{M L}_{\mathfrak{c}}^{\#}(F)$ the set of such $\left.(\lambda, \eta)\right)$ on $F$. Finally we can define the enhanced measure spectrum

$$
\mathrm{I}^{\#}: \mathcal{M L}_{\mathfrak{c}}^{\#}(F) \rightarrow \mathbb{R}^{r} \times \mathbb{R}_{+}^{\mathfrak{S}^{\prime}}
$$

such that:

for every $\gamma \in \mathfrak{S}^{\prime}$

$$
\mathrm{I}_{\gamma}^{\#}(\lambda, \eta)=\mathrm{I}_{\gamma}(\lambda)
$$

for every peripheral loop $C_{i}$.

$$
\mathrm{I}_{C_{i}}^{\#}(\lambda, \eta)=\eta_{i} \mathrm{I}_{C_{i}}(\lambda)
$$

Here is the enhanced version of Proposition 3.19.

Corollary 3.21. Let $F, F^{\prime} \in \mathcal{T}_{\mathfrak{c}}(S)$. Then there is a natural bijection

$$
\iota^{\#}: \mathcal{M L}_{\mathfrak{c}}(F)^{\#} \rightarrow \mathcal{M} \mathcal{L}_{\mathfrak{c}}\left(F^{\prime}\right)^{\#}
$$

such that for every (isotopy class of) simple closed curve $\gamma$ on $S$, we have

$$
\mathrm{I}_{\gamma}^{\#}((\lambda, \eta))=\mathrm{I}_{\gamma}^{\#}\left(\iota^{\#}(\lambda, \eta)\right) .
$$

Proposition 3.22. (i) The enhanced spectrum $\mathrm{I}^{\#}$ realizes an embedding of every $\mathcal{M} \mathcal{L}_{\mathfrak{c}}(F)^{\#}$ into $\mathbb{R}^{r} \times \overline{\mathbb{R}}_{+}^{\mathfrak{S}^{\prime}}$. Only the empty lamination goes to 0 . The image is homeomorphic to $\mathbb{R}^{6 g-6+3 r}$. The image of $\mathcal{M L}_{\mathfrak{c}}(F)^{\#, 0}$ (that is the set of enhanced laminations that do not enter any cusp) is homeomorphic to $\mathbb{R}^{6 g-6+2 r+r_{\mathcal{H}}}$

(ii) For every pant decomposition $\mathcal{D}$ of $\bar{\Sigma}$, consider the subset [already considered to deal with the length spectrum]

$$
\mathfrak{S}_{\mathcal{D}}=\left\{C_{1}, \cdots, C_{r}, z_{1}, z_{1}^{\prime}, z_{1}^{\prime \prime}, \cdots, z_{3 g-3+r}, z_{3 g-3+r}^{\prime}, z_{3 g-3+r}^{\prime \prime}\right\} \subset \mathfrak{S} .
$$

The projection onto this finite set of factors is already an embedding of $\mathcal{M} \mathcal{L}_{\mathfrak{c}}(F)^{\#}$. By varying $\mathcal{D}$ we get an atlas of a $P L$ structure on $\mathcal{M} \mathcal{L}_{\mathfrak{c}}(F)^{\#}$ (i.e. on $\mathbb{R}^{6 g-6+3 r}$ ). Similar facts hold for the restriction to $\mathcal{M L}_{\mathfrak{c}}(F)^{\#, 0}$.

(iii) Finite laminations are dense in $\mathcal{M L}_{\mathfrak{c}}(F)^{\#}\left(\mathcal{M L}_{\mathfrak{c}}(F)^{\#, 0}\right)$. 
(iv) For every $F, F^{\prime} \in \mathcal{T}_{\mathfrak{c}}(S)$, there is a canonical identification between the respective sets of finite enhanced measured geodesic laminations, and this extends to a canonical $P L$ isomorphism between $\mathcal{M L}_{\mathfrak{c}}(F)^{\#}$ and $\mathcal{M} \mathcal{L}_{\mathfrak{c}}\left(F^{\prime}\right)^{\#}$, which respects the ray structures. Similarly for $\mathcal{M} \mathcal{L}_{\mathfrak{c}}(\cdot)^{\#, 0}$.

Proof : We will sketch the proof of this proposition. We assume that the result is known when $S$ is compact (see [14](1), 25]). Thanks to Proposition 3.21 it is enough to deal with $F$ without cusps. Then the double $D F$ of $F^{\mathcal{C}}$ is compact, and we consider on $D F$ the involution $\tau$ that exchange the two copies of $F$. Let us denote by $M L(F)$ the set of $\tau$-invariant measured geodesic laminations on $D F$ that do not contain any component of $\partial F^{\mathcal{C}}$. The idea is to construct a map

$$
T: \mathcal{M L}_{\mathfrak{c}}(F) \rightarrow M L(F)
$$

that is surjective and such that

(1) the fiber over a lamination $\lambda^{\prime} \in M L(F)$ consists of $2^{k}$ laminations of $\mathcal{M} \mathcal{L}_{\mathfrak{c}}(F)$, where $k$ is the number of boundary components of $F^{\mathcal{C}}$ that intersect the support of $\lambda^{\prime}$. (2) For every $\lambda \in \mathcal{M L}_{\mathfrak{c}}(F)$, the restrictions to $\mathfrak{S}$ of both the spectrum of $T(\lambda)$ and of $\lambda$ coincide.

The existence of the map $T$ and the known results in the special cases recalled above will imply the Proposition.

The construction of the map $T$ runs as follows. Let $\lambda=\lambda_{b} \cup \lambda_{c} \in \mathcal{M} \mathcal{L}_{\mathfrak{c}}(F)$ be decomposed as above. We define $T\left(\lambda_{c}\right)$ to be the double of $\lambda_{c}$ in $D F$. For each leaf $l_{i}$ of $\lambda_{b}$, take a "big" segment $J_{i} \subset l_{i}$, and complete it to a simple arc $l_{i}^{\prime}$ properly embedded in $\left(F^{\mathcal{C}}, \partial F^{\mathcal{C}}\right)$, obtained by going straightly from each end-point of $J_{i}$ to the corresponding boundary component along an orthogonal segment. Clearly the double of $l_{i}^{\prime}$ is a simple non-trivial curve in $D F$, so there is a geodesic representative, say $c_{i}$, that is $\tau$-invariant and simple. Since $l_{i} \cap l_{j}=\varnothing$ the same holds for the $c_{i}$ 's. Moreover, since $l_{i} \cap \lambda_{c}=\varnothing$, the intersection of $c_{i}$ with $T\left(\lambda_{c}\right)$ is also empty. So we can define

$$
T(\lambda)=T\left(\lambda_{b}\right) \cup\left(c_{1}, a_{1}\right) \cup\left(c_{2}, a_{2}\right) \cup \ldots \cup\left(c_{n}, a_{n}\right) .
$$

where $a_{i}$ is the initial weight of $l_{i}$. This map satisfies (2) by construction; moreover, it follows from Corollary 3.20 that (1) holds for every $\lambda^{\prime}$ belonging to the image of $T$. The only point to check is that the map is surjective. The key remark is that for every $\lambda^{\prime} \in M L(F)$, every leaf $l$ hitting $\partial F^{\mathcal{C}}$ is necessarily closed. As it is $\tau$-invariant, then $l$ is orthogonal to $\partial F^{\mathcal{C}}$, and if $l$ intersects $\partial F^{\mathcal{C}}$ twice, then it is closed. Suppose that $l$ is a geodesic line, so that $l$ meets $\partial F$ exactly once. On the other hand, we know that the closure of $l$ is a minimal sublamination $\lambda^{\prime \prime}$, such that every leaf is dense in it. Thus if $l^{\prime \prime} \neq l$ is another leaf in $\lambda^{\prime \prime}$, then it intersects $\partial F^{\mathcal{C}}$ in a point $p$. Since $l$ is dense in $\lambda^{\prime \prime}$, there is a sequence of points in $l \cap \partial F^{\mathcal{C}}$ converging to $p$ and this contradicts the assumption that $l$ intersects $\partial F^{\mathcal{C}}$ once.

Thus a lamination in $M L(F)$ is given by the double of a compact lamination $\lambda_{c}$ in $F$ and of a finite number of weighted simple geodesics arcs in $F$ hitting orthogonally $\partial F^{\mathcal{C}}$. These arcs can be completed to give a family of simple arcs on $\hat{S}$ with end-points on $V$. Fix a signature on the end-points of such arcs. Finally we can apply Proposition 3.18 to these data and we get a suitable $\lambda=\lambda_{b} \cup \lambda_{c} \in \mathcal{M L}_{\mathfrak{c}}(F)$ such that $T(\lambda)=\lambda^{\prime}$. 
Finally we can define the map

$$
\mathfrak{p}_{\mathfrak{c}}^{\#}: \mathcal{M} \mathcal{L}_{\mathfrak{c}}(S)^{\#} \rightarrow \mathcal{T}_{\mathfrak{c}}^{\#}(S) .
$$

The total space is defined as the set of pairs

$$
((F, \epsilon),(\lambda, \eta))
$$

such that

(1) $(F, \epsilon)=\left(F, \epsilon_{1}, \ldots, \epsilon_{r}\right) \in \mathcal{T}_{\mathfrak{c}}(S)^{\#}$;

(2) $(\lambda, \eta)=\left(\lambda, \eta_{1}, \ldots, \eta_{r}\right) \in \mathcal{M L}_{\mathfrak{c}}(F)^{\#}$

Clearly

$$
\phi^{\#} \circ \mathfrak{p}^{\#}=\mathfrak{p} \circ \phi_{\mathcal{M L}}^{\#}
$$

where $\phi_{\mathcal{M L}}^{\#}$ denotes the forgetting projection of $\mathcal{M L}_{\mathfrak{c}}(S)^{\#}$ onto $\mathcal{M L}_{\mathfrak{c}}(S)$. We are going to see that in fact $\mathfrak{p}_{\mathfrak{c}}$ determines a bundle of enhanced lamination, that admits furthermore natural trivializations $\boldsymbol{t}$. It follows from the previous discussion that the image of $\mathrm{I}^{\#}$ does not depend on the choice of $F$, hence $\mathrm{I}^{\#}(S)$ is well defined. We want to define a natural bijection

$$
\mathfrak{t}: \mathcal{T}_{\mathfrak{c}}^{\#}(S) \times \mathrm{I}^{\#}(S) \rightarrow \mathcal{M} \mathcal{L}_{\mathfrak{c}}(S)^{\#} .
$$

For every $\xi \in \mathrm{I}^{\#}(S)$ and $F \in \mathcal{T}_{\mathfrak{c}}(S)$ there is a unique $(\lambda(\xi), \eta(\xi)) \in \mathcal{M L}_{\mathfrak{c}}(F)^{\#}$ that realizes $\xi$. So, let us put

$$
\mathfrak{t}(F, \epsilon, \xi)=\left(F, \epsilon, \rho_{\epsilon}(\lambda(x i), \eta(\xi)) .\right.
$$

It follows from the previous discussion that $\mathfrak{t}$ is a bijection. We stipulate that it is a homeomorphism, determining by the way a topology on $\mathcal{M L}_{\mathfrak{c}}(S)^{\#}$. Summing up, the map

$$
\mathfrak{p}^{\#}: \mathcal{M} \mathcal{L}_{\mathfrak{c}}(S)^{\#} \rightarrow \mathcal{T}_{\mathfrak{c}}(S)^{\#}
$$

can be considered as a canonically trivialized fiber bundle having both the base space and the fiber (analytically or PL) isomorphic to $\mathbb{R}^{6 g-6+3 r}$. Different choices of the base surface $F_{0}$ lead to isomorphic trivializations, via isomorphisms that preserve all the structures. These trivializations respect the ray structures. When $S$ is compact this specializes to the trivialized bundle $\mathcal{T}_{g} \times \mathcal{M L}_{g} \rightarrow \mathcal{T}_{g}$ mentioned in the Introduction.

Remark 3.23. The definition of $\mathfrak{t}$ could appear a bit distressing at a first sight. However the geometric meaning is simple. Given a spectrum of positive numbers, this determines the lamination up to choosing the way of spiraling towards the boundary components. If we give a sign to the elements of the spectrum corresponding to the boundary components, this allows to reconstruct the lamination by the rule: if the sign is positive the lamination spiral in the positive way, if the sign is negative the lamination spirals in the negative way with respect to a fixed orientation of the boundary component. In the non-enhanced set up, we have stipulated to use the boundary orientation induced by the surface one. Since the elements of an enhanced Teichmüller space can be regarded as hyperbolic surfaces equipped with an (arbitrary) orientation on each boundary component, it seems natural to reconstruct the lamination from the spetrum I ${ }^{\#}$ by means of such boundary component orientations.

This choice is suitable in view of the earthquake flow that we are going to define on $\mathcal{T}_{\mathfrak{c}}(S)^{\#}$. 
RICCARDO BENEDETTI AND FRANCESCO BONSANTE

3.6. Grafting, bending, earthquakes. Let $(F, \lambda) \in \mathcal{M L}(S)$. Grafting $(F, \lambda)$ produces a deformation $G r_{\lambda}(F)$ of $F$ in $\mathcal{P}(S)$, the Teichmüller-like space of complex projective structures (i.e. $\left(S^{2}, P S L(2, \mathbb{C})\right.$-structures) on $S$.

3-dimensional hyperbolic bending produces the $H$-hull of $G r_{\lambda}(F)$, that is, in a sense, its "holographic image" in $\mathbb{H}^{3}$.

The left (right) earthquake produces (in particular) a new element $\beta_{\lambda}^{L}(F)\left(\beta_{\lambda}^{R}(F)\right)$ in $\widetilde{\mathcal{T}}(S)$.

We will see in Section 4 how these constructions are materialized within the canonical Wick rotation-rescaling theory for $M G H$ Einstein spacetimes. For example, the grafting is eventually realized by the level surfaces of the cosmological times; earthquakes are strictly related to the Anti de Sitter bending procedure.

Here we limit to recall a few details about earthquakes, purely in terms of hyperbolic geometry.

Features of arbitrary $(F, \lambda)$. In such a general case, the leaves of $\lambda$ possibly enter the crowns of $F$. If $F$ is of finite area (see Lemma 3.5), basically the conclusions of Proposition 3.13 still hold. The only new fact is that possibly there is a finite number of isolated geodesic lines of $\lambda$ having at least one end converging to a point of some $\mathcal{E}_{\infty}$

The situation is quite different if $F$ is of infinite area. The set of isolated geodesic lines of $\lambda$ that are not entirely contained in one crown $\mathcal{E}$ is always finite. On the other hand, (1), (2), (3) and (5) of Proposition 3.13 definitely fails. For example, the support of a lamination $\lambda$ could contain bands homeomorphic to $[0,1] \times \mathbb{R}$, such that every $\{t\} \times \mathbb{R}$ maps onto a geodesic line of $\lambda$. Both ends of every such a line converge to some $\mathcal{E}_{\infty}$. We can also construct transverse measures such that $L_{W}$ is dense in such bands. This also shows that in general $\mathcal{L}_{S}$ is strictly contained in $\mathcal{L}_{W}$.

In general the fibers of I are, in any sense, infinite dimensional. For example we have:

Lemma 3.24. $\mathrm{I}^{-1}(0) \subset \mathcal{M L}(F)$ consists of laminations such that the support is entirely contained in the union of crowns.

On the other hand, the image of I is tame, in fact:

Proposition 3.25. $\mathrm{I}(\mathcal{M L}(F))=\mathrm{I}\left(\mathcal{M L}_{\mathfrak{c}}(\mathcal{K}(F))\right.$.

Earthquakes along finite laminations of $\mathcal{M L}_{\mathfrak{c}}(F)$. As finite laminations are dense, and arbitrary laminations $\lambda \in \mathcal{M} \mathcal{L}_{\mathfrak{c}}(F)$ look like finite ones at cusps and boundary components of $F^{\mathcal{C}}$, it is important (and easy) to understand earthquakes in the finite case.

Example 3.26. Let us consider again the Examples 3.15. Let $(F(l, t)$ be such that all twist parameters are strictly positive. Then, by definition $(F(l, t), \lambda(t))$ is obtained from $(F(l, 0), \lambda(t))$ via a left earthquake (along the measured geodesic lamination $\lambda(t)$ on $F(l, 0)) .(F(l,-t), \lambda(t))$ is obtained from $(F(l, 0), \lambda(t))$ via a right earthquake (along the measured geodesic lamination $\lambda(t)$ on $F(l, 0))$. In the reverse direction, $(F(l, 0), \lambda(t))$ is obtained from $(F(l, t), \lambda(t))$ via a right earthquake, and so on. This pattern of earthquakes does preserve the types. 
Similarly, let $F(s)$ be such that all shear parameters are strictly positive. Then, by definition $(F(s), \lambda(s))$ is obtained from $(F(0), \lambda(s)$ ) via a left earthquake (along the measured geodesic lamination $\lambda(s)$ on $F(0))$. $(F(-s), \lambda(s))$ is obtained from $(F(0), \lambda(s))$ via a right earthquake (along the measured geodesic lamination $\lambda(s)$ on $F(0)$ ). In the reverse direction, $(F(0), \lambda(s))$ is obtained from $(F(s), \lambda(s))$ via a right earthquake, and so on. This pattern does not preserve the types, for $F(0) \in \mathcal{T}_{g, r}$, while $F(s)$ is without cusps. Moreover, $\lambda(s)$ has the following special property:

For every boundary component $C_{i}$ of $F(s)^{\mathcal{C}}$

$$
l_{C_{i}}(F(s))=\mathrm{I}_{C_{i}}(\lambda(s)) .
$$

For every $(F, \lambda) \in \mathcal{M} \mathcal{L}_{\mathfrak{c}}(S), \lambda$ being finite, the definition of $\left(F^{\prime}, \lambda^{\prime}\right)$ obtained from $(F, \lambda)$ via a left (right) earthquake extends verbatim the one of the above examples, so that $\left(F^{\prime}, \lambda^{\prime}\right) \in \mathcal{M L}_{\mathfrak{c}}(S), \lambda^{\prime}$ is also a finite lamination, and $(F, \lambda)$ is obtained from $\left(F^{\prime}, \lambda^{\prime}\right)$ via the inverse right (left) earthquake.

Quake cocycles and general earthquakes. It is convenient to describe earthquakes by lifting everything to the universal covering. Let us set as usual

$$
\overline{\mathcal{K}}(\hat{F}) \subset F^{\mathcal{C}} \subset \hat{F}=\mathbb{H}^{2} / \Gamma
$$

Then $F^{\mathcal{C}}$ lifts to a $\Gamma$-invariant straight convex set $H$ of $\mathbb{H}^{2}$ (i.e $H$ is the closed convex hull of an ideal subset of $S_{\infty}^{1}$ ), and $\lambda$ lifts to a $\Gamma$-invariant measured geodesic lamination on $\stackrel{\circ}{H}$, that, for simplicity, we still denote by $\lambda$. If $F \in \mathcal{T}_{\mathfrak{c}}$, then $\overline{\mathcal{K}}(\hat{F})=F^{\mathcal{C}}$.

Lemma 3.27. Let $(F, \lambda) \in \mathcal{M L}_{\mathfrak{c}}(S)$ such that $\lambda$ is finite. Then there exists a left-quake cocycle

such that

$$
B_{\lambda}^{L}: \stackrel{\circ}{H} \times \stackrel{\circ}{H} \rightarrow P S L(2, \mathbb{R})
$$

(1) $B_{\lambda}^{L}(x, y) \circ B_{\lambda}^{L}(y, z)=B_{\lambda}^{L}(x, z)$ for every $x, y, z \in \stackrel{\circ}{H}$.

(2) $B_{\lambda}^{L}(x, x)=I d$ for every $x \in \stackrel{\circ}{H}$.

(3) $B_{\lambda}^{L}$ is constant on the strata of the stratification of $\stackrel{\circ}{H}$ determined by $\lambda$.

(4) $B_{\lambda}(\gamma x, \gamma y)=\gamma B_{\lambda}(x, y) \gamma^{-1}$, for every $\gamma \in \Gamma$.

(5) For every $x_{0}$ belonging to a 2-stratum of $\stackrel{\circ}{H}$,

$$
\stackrel{\circ}{H} \ni x \mapsto B_{\lambda}^{L}\left(x_{0}, x\right) x \in \mathbb{H}^{2}
$$

lifts the left earthquake $\beta_{\lambda}^{L}(F)$ to $\stackrel{\circ}{H}$. This cocycle is essentially unique. There exists a similar right-quake cocycle $B_{\lambda}^{R}$.

The proof is easy and the earthquake is equivalently encoded by its cocycle. For a general $(F, \lambda)$ we look for (essentially unique) quake-cocycles that satisfy all the properties of the previous Lemma, with the exception of the last one, and requiring furthermore that

(*) If $\lambda_{n} \rightarrow \lambda$ on a $\epsilon$-neighbourhood of the segment $[x, y]$ and $x, y \notin L_{W}$, then $B_{\lambda_{n}}(x, y) \rightarrow$ $B_{\lambda}(x, y)$.

Given such cocycles we can use the map of (5) in the previous Lemma as the general definition of earthquakes. 
For example, if $(F, \lambda) \in \mathcal{M} \mathcal{L}_{\mathfrak{c}}(S)$ the cocycle can be derived by using Lemma 3.27, the density of finite laminations and the fact that we require $(*)$. If $\left(F^{\prime}, \lambda^{\prime}\right)$ results from the left earthquake starting at $(F, \lambda)$, then this last belongs to $\mathcal{M L}_{\mathfrak{c}}(S)$ and $(F, \lambda)$ is obtained from it via the inverse right earthquake.

In fact, in [24] Epstein-Marden defined these quake-cocycles in general (extending the construction via finite approximations). Strictly speaking they consider only the case of (arbitrary) measured geodesic laminations on $\mathbb{H}^{2}$, but the same arguments holds for laminations on arbitrary straight convex sets $H$ - see also [10] for more details. Hence general left (right) earthquakes

$$
\left(F^{\prime}, \lambda^{\prime}\right)=\beta^{L}(F, \lambda)
$$

so that

$$
(F, \lambda)=\beta^{R}\left(F^{\prime}, \lambda^{\prime}\right)
$$

are eventually defined for arbitrary $(F, \lambda) \in \mathcal{M L}(S)$. We will also write $F^{\prime}=\beta_{\lambda}^{L}(F)$, $\lambda^{\prime}=\beta_{\lambda}^{L}(\lambda)$.

Earthquake flows on $\mathcal{M L}_{\mathfrak{c}}(S)$. Let $\lambda \in \mathcal{M L}_{\mathfrak{c}}(F)$. Consider the ray $(F, t \lambda), t \in$ $[0,+\infty)$. Then, for every $t>0$, set

$$
\left(F_{t}, \lambda_{t}\right)=\left(\beta_{t \lambda}^{L}(F), \frac{1}{t} \beta_{t \lambda}^{L}(t \lambda)\right), \quad t \geq 0 .
$$

This continuously extends at $t=0$ by

$$
\left(F_{0}, \lambda_{0}\right)=(F, \lambda)
$$

We have

$$
\left(\left(F_{t}\right)_{s},\left(\lambda_{t}\right)_{s}\right)=\left(F_{t+s}, \lambda_{t+s}\right)
$$

hence this defines the so called left-quake flow on $\mathcal{M L}_{\mathfrak{c}}(S)$. In particular this allows to define a sort of "exponential" map

$$
\psi^{L}: \mathcal{M L}_{\mathfrak{c}}(F) \rightarrow \mathcal{M L}_{\mathfrak{c}}(S)
$$

by evaluating the flow at $t=1$. We do similarly for the right-quake flow.

Let $p_{i} \in V$ and $C_{i}$ be the curve surrounding it; as $\mathrm{I}_{C_{i}}(t \lambda)=t \mathrm{I}_{C_{i}}(\lambda)$, there is a unique "critical value" $t_{i}$ (see below) such that $\mathrm{I}_{C_{i}}(t \lambda)=l_{C_{i}}(F)$.

For every $t$, we denote by $l(t)$ the marked length spectrum of $F_{t}$, by $\theta(t)$ its type, by $\mathrm{I}(t)$ the marked measure spectrum of $\lambda_{t}$, by $\sigma_{t}: V \rightarrow\{ \pm\}$ its signature, and so on. The following Lemma describes the behaviour of these objects along the flow.

Lemma 3.28. The marked measure spectrum is constant for every $t$, that is

$$
\mathrm{I}_{\gamma}(t)=\mathrm{I}_{\gamma}(0) \text { for every } \gamma \in \mathfrak{S} .
$$

Let $p_{i} \in V$ and $C_{i}$ be the curve surrounding it.

If $p_{i} \in V_{\mathcal{H}}(0)$, then:

$$
l_{C_{i}}(t)=\left|l_{C_{i}}(0)-t \sigma_{i}(\lambda) \mathrm{I}_{C_{i}}(0)\right|
$$

and

$$
\sigma_{i}(t)=\operatorname{sign}\left[l_{C_{i}}-t \sigma_{i}(\lambda) \mathrm{I}_{C_{i}}(0)\right] \sigma_{i}(0)
$$


If $p_{i} \in V_{\mathcal{P}}(0)$ then:

$$
l_{C_{i}}(t)=t \mathrm{I}_{C_{i}}(0)
$$

and

$$
\sigma_{i}(t)=-1
$$

As every $\lambda \in \mathcal{M} \mathcal{L}_{\mathfrak{c}}(F)$ looks finite at cusps and boundary components of $F^{\mathcal{C}}$, it is enough (and fairly easy) to check the Lemma in the finite case, by using also Examples 3.26 .

Remark 3.29. If $p_{i} \in V_{\mathcal{P}}$ and the lamination enters the corresponding cusp, then for $t>0$ the cusp opens on a geodesic boundary component whose length linearly depends on $t$ with slope equal to $\mathrm{I}_{C_{i}}(0)$. The way of spiraling of $\lambda_{t}$ around $p_{i}$ is always negative (positive for right earthquakes).

Let us consider more carefully the case $p_{i} \in V_{\mathcal{H}}$. Notice that if $\lambda$ does not spiral around $C_{i}$ then the length of $C_{i}$ is constant. In the other cases let us distinguish two possibilities according to the sense of spiraling of $\lambda$.

(1) Case $\sigma_{i}(0)=-1$. Then for every $t>0$,

$$
\sigma_{i}(t)=-1, \quad l_{C_{i}}=l_{C_{i}}(0)+t \mathrm{I}_{C_{i}}(0) .
$$

Thus the length of $C_{i}$ increases linearly of slope $\mathrm{I}_{C_{i}}(0)$ and the laminations continues to spiral in the negative direction.

(2) Case $\sigma_{i}(0)=1$. There is a critical time $t_{i}=l_{C_{i}}(0) / \mathrm{I}_{C_{i}}(0)$. Before $t_{i}$ the length of $C_{i}$ decreases linearly and the lamination spiral in the positive direction. At $t_{i}, C_{i}$ is become a cusp. After $t_{i}, C_{i}$ is again a boundary component but the way of spiraling is now negative.

Remark 3.30. The above Proposition points out in every $\mathcal{M} \mathcal{L}_{\mathfrak{c}}(F)$ the set :

$$
\mathcal{V}_{\mathfrak{c}}(F)=\left\{\lambda ; \mathrm{I}_{C_{i}}(\lambda)<l_{C_{i}}(F) ; i \in V_{\mathcal{H}}\right\} .
$$

Note that this set is not preserved by the canonical bijections stated in Proposition 3.22 (iv).

Corollary 3.31. The restriction of the exponential-like map $\psi^{L}$ to $\mathcal{V}_{\mathfrak{c}}(F) \cap \mathcal{M} \mathcal{L}_{\mathfrak{c}}(F)^{0}$ preserves the type and the signatures. The restriction of this map to the whole of $\mathcal{V}_{\mathfrak{c}}(F)$ has generic image over the top-dimensional cell of $\mathcal{T}_{\mathfrak{c}}(S)$.

The quake-flow on $\mathcal{M} \mathcal{L}_{\mathfrak{c}}(S)^{\#}$. We will define an earthquake flow on $\mathcal{M} \mathcal{L}_{\mathfrak{c}}(S)^{\#}$ that will satisfy the following properties

(1) $\beta_{t}^{\#} \circ \beta_{s}^{\#}=\beta_{t+s}^{\#}$.

(2) Every flow line $\left\{\beta_{t}^{\#}(F, \epsilon, \lambda, \eta) \mid t>0\right\}$ is horizontal with respect to the trivialization of $\mathcal{M L}_{\mathfrak{c}}^{\#}(S)$. This means that the enhanced lamination is constant along the flow.

(3) If we include $\mathcal{M L}_{\mathfrak{c}}(S)$ into $\mathcal{M} \mathcal{L}_{\mathfrak{c}}(S)^{\#}$ by sending $(F, \lambda)$ to $(F, \epsilon, \lambda, \eta)$ with $\epsilon_{i}=1$ for every $i$ and $\eta_{i}=1$ for every $i \in V_{\mathcal{P}}$ then $\beta=\phi_{\mathcal{M L}}^{\#} \circ \beta^{\#}$ (where $\phi_{\mathcal{M L}}^{\#}$ is the usual forgetting map).

Remark 3.32. Before giving the actual definition, we describe the qualitative idea. Earthquakes paths on $\mathcal{T}_{\mathfrak{c}}(S)$ rebounce when reaches a cusp. Since $\mathcal{T}_{\mathfrak{c}}(S)^{\#}$ is obtained by reflecting $\mathcal{T}_{\mathfrak{c}}(S)$ along its faces, it is natural to lift such a paths to horizontal paths 
on $\mathcal{T}_{\mathfrak{c}}(S)^{\#}$. Instead of rebouncing the enhanced lamination after a cusp is obtained by a reflection along a boundary component of the initial lamination. This liftings are unique (up to the choice of a initial signature $\epsilon$ ) when $F$ does not contain cusp. When $F$ contains a cups then there are many possible liftings due to the possible choices of the signature of the cusp after the earthquake. Thus data $(F, \epsilon, \lambda)$ are not sufficient to determines the lifting. On the other hand the information of a signature of $\lambda$ around the cusp solves this ambiguity.

Let us come to the actual definition:

$$
\beta_{t}^{\#}(F, \epsilon, \lambda, \eta)=(\bar{F}, \bar{\epsilon}, \bar{\lambda}, \bar{\eta})
$$

where

(a) Similarly to the definition of the map $\mathfrak{t},(\bar{F}, \bar{\lambda})=\beta\left(F, \rho_{\epsilon}(\lambda)\right)$;

(b) $\bar{\epsilon}_{i}=\epsilon_{i} \operatorname{sign}\left(l_{C_{i}}(F)+t \eta_{i} \mathrm{I}_{C_{i}}(\lambda)\right)$.

(c) $\bar{\eta}_{i}=\eta_{i} \operatorname{sign}\left(l_{C_{i}}(F)+t \eta_{i} \mathrm{I}_{C_{i}}(\lambda)\right)$.

Property (1) follows from the fact that $\beta$ is a flow. Point (2) depends on the fact the spectrum of $\lambda_{t}$ is constant and the products $\epsilon_{i}(t) \eta_{i}(t)$ are constant. Point (3) is straightforward. The only point to check is that $\beta^{\#}$ is continuous, as a map $\mathbb{R}_{>} 0 \times$ $\mathcal{M L}_{\mathfrak{c}}^{\#}(S) \rightarrow \mathcal{M L}_{\mathfrak{c}}^{\#}(S)$. By the definition of the topology of $\mathcal{M L}_{\mathfrak{c}}^{\#}(S)$ it is enough to show that for every $\gamma \in \mathfrak{S}$ the functions

$$
(t,(F, \epsilon, \lambda, \eta)) \mapsto l_{\gamma}^{\#}\left(\beta_{t}^{\#}(F, \epsilon, \lambda, \eta)\right) \quad(t,(F, \epsilon, \lambda, \eta)) \mapsto \mathrm{I}_{\gamma}^{\#}\left(\beta_{t}^{\#}(F, \epsilon, \lambda, \eta)\right)
$$

are continuous. If $\gamma$ is not peripheral, then $l_{\gamma}^{\#}\left(\beta_{t}^{\#}(F, \epsilon, \lambda, \eta)\right)$ and $\mathrm{I}_{\gamma}^{\#}\left(\beta^{\#}(t, F, \epsilon, \lambda, \eta)\right.$ depend only on $F$ and $\lambda$ so the continuity is a consequence of the continuity of $\beta$.

If $\gamma$ is peripheral, then by Lemma 3.28 we have

$$
\begin{aligned}
& l_{\gamma}^{\#}\left(\beta_{t}^{\#}(F, \epsilon, \lambda, \eta)\right)=l_{\gamma}^{\#}(F, \epsilon)-t \mathrm{I}_{\gamma}^{\#}(F, \epsilon, \lambda, \eta) \\
& \mathrm{I}_{\gamma}^{\#}\left(\beta_{t}^{\#}(F, \epsilon, \lambda, \eta)\right)=\mathrm{I}_{\gamma}^{\#}(F, \epsilon) .
\end{aligned}
$$

For every $\xi \in \mathrm{I}^{\#}(S)$ let us consider the map of $\mathbb{R}_{\geq 0} \times \mathcal{T}_{\mathfrak{c}}^{\#}(S) \rightarrow \mathcal{T}_{\mathfrak{c}}^{\#}(S)$ that associates to $t,(F, \epsilon)$ the projection on $\mathcal{T}_{\mathfrak{c}}^{\#}(S)$ of $\beta_{t}(F, \epsilon, \xi(F)$ ) (where $\xi(F)$ is the realization of $\xi$ with respect to the structure given by $F)$. By $(2)$ it is a flow on $\mathcal{T}_{\mathfrak{c}}(S)^{\#}$. We will denote by $\mathcal{E}_{\xi}^{\#}$ the homeomorphism of $\mathcal{T}_{\mathfrak{c}}(S)^{\#}$ corresponding to such a flow at time 1 (notice that $\mathcal{E}_{\xi} \circ \mathcal{E}_{\xi}=\mathcal{E}_{2 \xi}$ ), it will be called the enhanced earthquake along $\xi$.

\section{Earthquake Theorem.}

Theorem 3.33. [Earthquake Theorem on $\mathcal{T}_{\mathfrak{c}}(S)$ ] For every $F_{0}, F_{1} \in \mathcal{T}_{\mathfrak{c}}(S)$, denote by $m$ the number of points in $V$ that do not correspond to cusp of $F_{1}$ nor of $F_{2}$. Then there exist exactly $2^{m}$ left earthquakes such that $F_{1}=\beta_{\lambda}^{L}\left(F_{0}\right)$. The similar statement holds with respect to right-quakes.

This is a consequence of the somewhat more precise

Theorem 3.34. [Earthquake Theorem on $\mathcal{T}_{\mathfrak{c}}(S)^{\#}$ ] For every $\left(F_{0}, \epsilon_{0}\right),\left(F_{1}, \epsilon_{1}\right) \in \mathcal{T}_{\mathfrak{c}}(S)^{\# \text {, }}$ there is a unique $\xi \in \mathrm{I}^{\#}(S)$ such that $\mathcal{E}_{\xi}^{\#}\left(F_{0}, \epsilon_{0}\right)=\left(F_{1}, \epsilon_{1}\right)$ Similarly for the right quakes.

Given two "signed" surfaces $\left(F_{0}, \sigma_{0}\right)$ and $\left(F_{1}, \sigma_{1}\right)$ in $\mathcal{T}_{\mathfrak{c}}(S)$, where the respective signatures are arbitrary maps $\sigma_{j}: V \rightarrow\{ \pm 1\}$ ), we say that they are left-quake compatible if 
there exists a left earthquake $\left(F_{1}, \lambda_{1}\right)=\beta^{L}\left(F_{0}, \lambda_{0}\right)$ such that $\sigma_{j}=\sigma_{\lambda_{j}}$. The following is an easy Corollary of Lemma 3.28 and of Theorem 3.33 .

Corollary 3.35. The signed surfaces $\left(F_{0}, \sigma_{0}\right)$ and $\left(F_{1}, \sigma_{1}\right)$ are left-quake compatible if and only if for every $i=1, \ldots, r$ the following condition is satisfied:

If $l_{C_{i}}\left(F_{1}\right)<l_{C_{i}}\left(F_{0}\right)$, then $\sigma_{0}(i)=1$. If $l_{C_{i}}\left(F_{1}\right)>l_{C_{i}}\left(F_{0}\right)$, then $\sigma_{1}(i)=1$.

Symmetric statements hold w.r.t. the right-quake compatibility.

In Section 5 we will outline an $A d S$ proof of the Earthquake Theorem (by following [19]) that generalizes Mess's proof in the special case of compact $S$.

$\mathcal{M L}_{\mathfrak{c}}(S)$ as tangent bundle of $\mathcal{T}_{\mathfrak{c}}(S)$. We have seen above that the bundle

$$
\mathfrak{p}^{\#}: \mathcal{M L}_{\mathcal{C}}(S)^{\#} \rightarrow \mathcal{T}_{\mathcal{C}}(S)^{\#}
$$

shares some properties with the tangent bundles $T \mathcal{T}_{\mathfrak{c}}^{\#}$ of its base space. We are going to substantiate this fact by means of quake-flows. In fact we have associated to every $\xi \in \mathrm{I}^{\#}(S)$ a flow of $\mathcal{T}_{\mathfrak{c}}(S)^{\#}$, So we can consider the infinitesimal generator of such a flow, that is a vector field on $\mathcal{T}_{\mathfrak{c}}(S)^{\#}$, say $X_{\xi}$.

Proposition 3.36. The map

$$
\Pi: \mathcal{T}_{\mathfrak{c}}(S)^{\#} \times \mathrm{I}^{\#}(S) \rightarrow T \mathcal{T}_{\mathfrak{c}}(S)^{\#}
$$

defined by $\Pi(\xi, F)=X_{\xi}(F)$ is a trivialization of $T \mathcal{T}_{\mathfrak{c}}(S)$.

As in the case of compact $S$, it is a consequence of the convexity of the length function along earthquakes paths.

Remark 3.37. The map $\Pi$ is only a topological trivialization. This means that the identifications between tangent spaces arising from $\Pi$ are not linear.

For a fixed type $\theta$, denotes by $\mathrm{I}^{\#}(S)^{\theta}$ the points corresponding to laminations that do not enter any cusp. It is clear that for a point $F \in \mathcal{T}_{\mathfrak{c}}^{\theta}(S)^{\#}$ we have that $X_{\xi}(F)$ is tangent to $T_{\mathfrak{c}}^{\theta}(S)^{\#}$. So we get that the restriction of $\Pi$ to $\mathcal{T}_{\mathcal{C}}^{\theta}(S)^{\#} \times \mathrm{I}^{\#}(S)^{\theta}$ is a trivialization of $T \mathcal{T}_{\mathfrak{c}}^{\theta}(S)^{\#}$.

\section{WICK ROTATION-RESCALING THEORY}

We refer to [10]. Let $S$ be a base surface of finite type. Recall from the Introduction and Section 2, that $\mathcal{M G H}_{\kappa}(S)$ denotes the Teichmüller-like space of Einstein maximal globally hyperbolic spacetimes of constant curvature $\kappa=0, \pm 1$, that contain a complete Cauchy surface homeomorphic to $S$.

Denote by $\mathcal{P}(S)$ the Teichmüller-like space of complex projective (that is $\left(S^{2}, P S L(2, \mathbb{C})\right.$ )manifold) structures on $S$. Here $S^{2}$ is the Riemann sphere, identified with $S_{\infty}^{2}=\partial \mathbb{H}^{3}$, and $\operatorname{PSL}(2, \mathbb{C}) \cong \operatorname{Isom}^{+}\left(\mathbb{H}^{3}\right)$.

The aim of this section is to illustrate the following pattern of statements (given here in somewhat informal way):

(Classifications) For every surface $S$ of finite type, and every $\kappa=0, \pm 1$, there are geometrically defined "materialization" maps

$$
\mathfrak{m}_{\mathcal{P}}: \mathcal{M L}(S) \rightarrow \mathcal{P}(S)
$$




$$
\mathfrak{m}_{\kappa}: \mathcal{M L}(S) \rightarrow \mathcal{M G H}_{\kappa}(S)
$$

that actually make $\mathcal{M L}(S)$ an universal parameter space.

(Canonical correlations) For every $(F, \lambda) \in \mathcal{M L}(S)$, there are geometrical correlations between the spacetimes $\mathfrak{m}_{\kappa}(F, \lambda)$ or between them and the projective surface $\mathfrak{m}_{\mathcal{P}}(F, \lambda)$. Such correlations are either realized by means of canonical rescalings or Wick rotations directed by the respective cosmological times, with universal rescaling functions.

Let us explain first some terms entering the last statement.

Definition 4.1. Let $(M, h)$ be any spacetime and $X$ be a nowhere vanishing $h$-timelike and future directed vector field on $M$. Let $\alpha, \beta: M \rightarrow \mathbb{R}_{+}$be positive functions.

We say that the Riemannian manifold $(M, g)$ is obtained from $(M, h)$ via the Wick rotation directed by $X$, with vertical (resp. horizontal) rescaling function $\beta$ (resp. $\alpha$ ), if for every $y \in M$, the $g$ - and $h$-orthogonal spaces to $X(y)$ coincide (denoted it by $\left.<X(y)>^{\perp}\right)$, and

$$
\begin{aligned}
\|X(y)\|_{g} & =-\beta(y)\|X(y)\|_{h} \\
\left.g\right|_{<X(y)>^{\perp}} & =\left.\alpha(y) h\right|_{<X(y)>^{\perp}} .
\end{aligned}
$$

Similarly, the spacetime $\left(M, h^{\prime}\right)$ is obtained from $(M, h)$ via the rescaling directed by $X$, with vertical (resp. horizontal) rescaling function $\beta$ (resp. $\alpha$ ), if for every $y \in M$, the $h^{\prime}$ - and $h$-orthogonal spaces to $X(y)$ coincide, and

$$
\begin{gathered}
\|X(y)\|_{h^{\prime}}=\beta(y)\|X(y)\|_{h} \\
\left.h^{\prime}\right|_{<X(y)>^{\perp}}=\left.\alpha(y) h\right|_{<X(y)>^{\perp} .} .
\end{gathered}
$$

4.1. Cosmological time. We refer to [2] for a general treatment of this matter. Here we limit ourselves to recalling the main features of this notion. Let $(M, h)$ be any spacetime. The cosmological function

$$
\tau: M \rightarrow(0,+\infty]
$$

is defined as follows: let $C^{-}(q)$ be the set of past-directed causal curves in $M$ that start at $q \in M$. Then

$$
\tau(q)=\sup \left\{L(c) \mid c \in C^{-}(q)\right\}
$$

where $L(c)$ denotes the Lorentzian length of $c$. Roughly speaking, this gives the (possibly infinite) proper time that every event $q \in M$ has been in existence in $M$. The function $\tau$ is said regular if it is finite valued for every $q \in M$, and $\tau \rightarrow 0$ along every past-directed inextensible causal curve. In such a case it turns that $\tau$ is a continuous global time on $M$, called its cosmological time. This cosmological time (if it exists) represents an intrinsic feature of the spacetime. Having cosmological time has strong consequences for the structure of $M$, and $\tau$ itself has stronger properties (it is locally Lipschitz and twice differentiable almost everywhere). In particular: $M$ is globally hyperbolic; for every $q \in M$, there exists a future-directed time-like unit speed geodesic ray whose length equals $\tau(q)$. Up to a suitable past-asymptotic equivalence, these rays form the initial singularity of $M$. In a sense $\tau$ gives the Lorentzian distance of every event from the initial singularity. 
4.2. Grafting and Lorentzian grafting. Before describing in some formal way how to get a parameterizations of $\mathcal{M G H}_{\kappa}(S)$ and $\mathcal{P}(S)$ in terms of $\mathcal{M L}(S)$, we will explain how to associate to a pair $(F, \lambda) \in \mathcal{M L}(S)$ a projective structure on $S$ and a spacetime of constant curvature $\kappa$, in some simple cases.

First consider the case $S$ compact closed and $\lambda$ empty. Given a hyperbolic structure $F=(S, h)$ on $S$, the projective structure associated to it, that, with a little abuse, we will denote simply by $F$, is the structure whose developing map coincides (up to post-composition with $g \in P S L(2, \mathbb{C})$ ) with the isometric developing map of $F$. Structures obtained in this way are called Fuchsian and are characterized by the following requirements:

(1) the developing map is injective,

(2) the holonomy is conjugated in $\operatorname{PSL}(2, \mathbb{R})$.

For the Lorentzian side, define $\mathfrak{m}_{\kappa}(F)$ to be the spacetime $\left(S \times I, g_{\kappa}\right)$ where $I$ is the interval $(0,+\infty)$ for $\kappa \geq 0$ and $I=(0, \pi / 2)$ for $\kappa=-1$ and $g_{\kappa}=g_{\kappa}(F)$ is so defined

$$
g_{\kappa}= \begin{cases}-d t^{2}+t^{2} h & \text { if } \kappa=0 \\ -d t^{2}+\operatorname{sh}^{2}(t) h & \text { if } \kappa=1 \\ -d t^{2}+\sin ^{2}(t) h & \text { if } \kappa=-1\end{cases}
$$

The fact that $g_{\kappa}$ has constant curvature $\kappa$ is just a local computation independent of the compactness of $F$. Thus one does the computation assuming $F=\mathbb{H}^{2}$. For instance, for $\kappa=0$, one embeds $\mathbb{H}^{2}$ in the Minkowski space $\mathbb{X}_{0}$ and take the normal evolution of $\mathbb{H}^{2}$ (that is a map $\mathbb{H}^{2} \times \mathbb{R}_{\geq} 0 \rightarrow \mathbb{X}_{0}$ sending $(x, t)$ to $t x$ ) the pull-back of the Minkowski metric takes the form (11).

Remark 4.2. Strictly speaking $\mathfrak{m}_{-1}(F)$ is not maximal. In fact the metric $g_{\kappa}$ can be defined as well on the interval $(0, \pi)$. On the other hand, for some reason that will appear clear it is better to define $\mathfrak{m}_{-1}(F)$ in this way and then to take its maximal extension.

Now suppose $S$ to be closed and $\lambda$ to be a weighted curve $(c, a)$. The projective surface $\mathfrak{m}_{\mathcal{P}}(F, \lambda)$ is the grafting of $F$ along $\lambda$, that we sometimes denote by $G r_{\lambda}(F)$. We cut $F$ along $c$ and grafts a projective annulus $A=c \times[0, a]$ whose developing map can be explicitly described in the following way. We can choose a developing map dev $: \tilde{F} \rightarrow \mathbb{H}^{2}$ such that $c$ lifts to a geodesic $\tilde{c}$ with end-points at 0 and $\infty$. The developing map of $A$ is given by

$$
\tilde{c} \times[0, a] \ni(x, t) \rightarrow \operatorname{dev}(x) e^{i t} \in \mathbb{C} \subset S^{2} .
$$

The fact that $A$ can be grafted on $F$ descends on the fact that the developing map of each component of $\partial A$ is conjugated in $P S L(2, \mathbb{C})$ to the developing map of $c$. Notice that $A$ carries a natural Euclidean metric. The length of each boundary component of $A$ is equal to the length of $c$ whereas the width of $A$ is equal to $a$. Thus we can consider on $G r_{\lambda}(F)$ the metric that is hyperbolic on $F \backslash c$ and Euclidean on $A$. Such a metric is $C^{1}$ and compatible with the conformal structure underlying the projective structure of $G r_{\lambda}(F)$. We call it the the Thurston metric of $G r_{\lambda}(F)$, in what follows we often indicate with $G r_{\lambda}(F)$ both the projective structure and the metric structure on $S$. 
Remark 4.3. Thurston distance is defined on every projective structure on $S$ and is a metric compatible with the conformal class of the projective surface. The interesting point showed by Thurston is that Thurston metric determines the projective structure. This means that a map between projective surfaces is a projective equivalence iff it is an isometry with respect to the corresponding Thusrton distances.

Remark 4.4. If $a$ is small, then the holonomy group of $G r_{\lambda}(F)$, say $\Gamma$, is quasiFuchsian and the developing map is injective with image a component of the discontinuity domain. Thus, $G r_{\lambda}(F)$ can be regarded as an asymptotic end of the quasi-Fucshian manifold $\mathbb{H}^{3} / \Gamma$. In fact, the boundary component of the convex core facing $G r_{\lambda}(F)$ is isometric to $F$ bent along $c$ with bending angle $a$, and the annulus $A$ coincides with the set of points in $G r_{\lambda}(F)$ that are sent by the retraction on the convex core to the bending line.

Moreover, let us consider the component of the complement of the convex core in $\mathbb{H}^{3} / \Gamma$, facing $G r_{\lambda}(F)$. Then the distance $d$ from the convex core is a $\mathrm{C}^{1}$ function on it whose level surfaces are isometric to $\operatorname{ch} d \cdot G r_{\operatorname{tgh} d \lambda}(F)$ (if $X$ is a metric space $\lambda \cdot X$ denotes the metric space obtained by multiplying the distance by $\lambda$ ).

Thurston generalized this idea and showed how to associate to each projective structure on $S$ a non-complete hyperbolic structure on $S \times(0,1)$, called the $H$-hull such that

(1) its completion is $S \times[0,1)$ and $S \times\{0\}$ is a locally convex bent surface $F$ along a lamination $\lambda$

(2) the asymptotic end $S \times\{1\}$ carries the original projective surface that in tunrs coincides with $G r_{\lambda}(F)$.

Moreover the distance $d$ from $S \times\{0\}$ is a $C^{1}$ function and level surfaces are isometric to

$$
\operatorname{ch} d \cdot G r_{\operatorname{tgh} d \lambda}(F) \text {. }
$$

Clearly in the quasi-Fuchsian case the $H$-hull is simply the end of the corresponding quasi-Fuchsian manifold facing the projective surface.

Consider now the Lorentzian case.

To construct $\mathfrak{m}_{\kappa}(F, \lambda)$ we will deform the structure on $\mathfrak{m}_{\kappa}(F)$ by means of a construction that is reminescent of the grafting procedure, so we call it the Lorentzian grafting.

With a little abuse let us denote by $c$ the geodesic representative of $c$ with respect to the hyperbolic structure $F$. Then one shows that the timelike surface $c \times I$ is totally geodesic in $\mathfrak{m}_{\kappa}(F)$ (it is still a local computation - for instance, in the flat case it is a direct consequence of the fact that geodesics of $\mathbb{H}^{2}$ are intersection of $\mathbb{H}^{2}$ with linear time-like planes of Minkowski space). Then one cuts $\mathfrak{m}_{\kappa}(F)$ along $c \times I$ and grafts a piece, say $\mathfrak{m}_{\kappa}(A)$ such that

(1) topologically $\mathfrak{m}_{\kappa}(A)=(c \times[0, a]) \times I$ that is a the product of the annulus $A=c \times[0, a]$ by the time interval $I$.

(2) the restriction of the metric on each slice $A \times\{t\}$ is a Euclidean annulus, whose width depends only on $a$ and on $t$ and whose boundary length is equal to the length of $c \times\{t\} \subset \mathfrak{m}_{\kappa}(F)$.

(3) the boundary of $\mathfrak{m}_{\kappa}(A)$ (that is $\partial A \times I=c \times I \times\{0, a\}$ ) is totally geodesic and each component is isometric to $c \times I$ 
For instance in the flat case $\mathfrak{m}_{0}(A)$ is just $(c \times I) \times[0, a]$ with the product metric (that is flat since it is the product of two flat metrics). For the other curvatures, the expression of the metric on $\mathfrak{m}_{0}(A)$ takes the more complicated form given by

$$
\begin{cases}-d t^{2}+\left(\operatorname{ch}^{2}(t) d r^{2}+\operatorname{sh}^{2}(t) d \theta^{2}\right) & \text { for } \kappa=1 \\ -d t^{2}+\left(\cos ^{2}(t) d r^{2}+\sin ^{2}(t) d \theta^{2}\right) & \text { for } \kappa=-1\end{cases}
$$

where $\theta$ is an arc parameter on $c$ and $r$ is the variable on $[0, a]$. Notice that the width of $A \times\{t\}$ is independent of $t$ only in the flat case.

Remark 4.5. The piece $\mathfrak{m}_{-1}(A)$ is well-defined only for $t \in(0, \pi / 2)$ and this explains the definition of $\mathfrak{m}_{-1}(F)$. In general the spacetime obtained for $\kappa=-1$ is never maximal, so more correctly $\mathfrak{m}_{-1}(F)$ will denote the maximal extension of the spacetime we have defined. In the next sections we will explain the reason of this asymmetry and also how the spacetime we have defined is uniquely determined by its maximal extension.

Remark 4.6. A way to define $\mathfrak{m}_{\kappa}(F, \lambda)$ for a generic $\lambda$ is by means of an approximation argument. We take a sequence of simple weighted curves $\lambda_{n}=\left(c_{n}, a_{n}\right)$ converging to $\lambda$ and define $\mathfrak{m}_{\kappa}(F, \lambda)=\lim \mathfrak{m}_{\kappa}\left(F, \lambda_{n}\right)$. Clearly the existence of this limit has to be checked: to this aim it is better to work in the framework of $(X, G)$-structures and study the behaviour of the developing maps of $\mathfrak{m}_{\kappa}(F, \lambda)$. This will be the theme of the next sections.

Notice that the construction of $\mathfrak{m}_{\kappa}(F, \lambda)$ gives, as a by-product, a natural foliation of the spacetime in spacelike surfaces homeomorphic to $S$. In fact in both $\mathfrak{m}_{\kappa}(F)$ and $\mathfrak{m}_{\kappa}(A)$ we have pointed out a time-function $t$ to express the metric in some explicit way, these functions glue to a time-function on $\mathfrak{m}_{\kappa}(F, \lambda)$. Notice however that the function $t$ in $\mathfrak{m}_{\kappa}(F, \lambda)$ is not smooth: its level surfaces are made by hyperbolic pieces and Euclidean annuli. In fact they are reminescent of the usual grafted surfaces.

Let us consider the flat case. In such a case the $t$ level surface corresponding to some value $t_{0}$ is obtained by multiplying the hyperbolic metric on $F$ by the factor $t_{0}^{2}$ (that is by multiplying the hyperbolic distance by the factor $t_{0}$ ), by cutting along $c$ and gluing a Euclidean annulus of width $a$. This is the same as grafting an annulus of width $a / t_{0}$ on $F$ and then multiplying the grafted distance by the factor $t_{0}$.

More generally one can check explicitly that for a weighted multi-curve $\lambda=(c, a)$ the surface $t^{-1}\left(t_{0}\right) \subset \mathfrak{m}_{\kappa}(F, \lambda)$ is metrically equal to

$$
\begin{array}{ll}
t_{0} \cdot G r_{\lambda / t_{0}}(F) & \text { if } \kappa=0 \\
\operatorname{sh} t_{0} \cdot G r_{\lambda / \operatorname{tgh} t_{0}}(F) & \text { if } \kappa=1 \\
\sin t_{0} \cdot G r_{\lambda / \tan t_{0}}(F) & \text { if } \kappa=-1
\end{array}
$$

The point that makes this remark interesting is that the function $t$ is the cosmological time of $\mathfrak{m}_{\kappa}(F, \lambda)$, so it is somehow independent of the parameterization and the same formulae to express the level surface work for every $(F, \lambda)$. This remark motivates the idea to find a canonical rescaling directed by the gradient of the cosmological time transforming $\mathfrak{m}_{0}(F, \lambda)$ into $\mathfrak{m}_{ \pm 1}(F, \lambda)$ and a Wick Rotation transforming $\mathfrak{m}_{0}(F, \lambda)$ into the $H$-hull of $G r_{\lambda}(F)$.

Remark 4.7. Consider the case $S$ of finite type. For $F \in \mathcal{T}_{\mathcal{C}}(S)$ we could try to define $\mathfrak{m}_{\kappa}(F)$ as in the closed case. Notice however that the slice $S \times\{t\}$ is in general not 
complete. In fact such a spacetime have a natural totally geodesic timelike boundary that is homeomorphic to $\partial F^{\mathcal{C}} \times I$. A way to get a complete level surface is then for each boundary component $c$ of $F$ to glue a piece $\mathfrak{m}_{\kappa}(\Delta)$ where $\Delta=c \times[0,+\infty)$ is a annulus with infinite width and $\mathfrak{m}_{\kappa}(\Delta)=\Delta \times I$ with a metric given in (3). Notice that the definition of $\mathfrak{m}_{\kappa}(F)$ is then consistent with the previous case provided to allow boundary component of $F$ to carry an infinite weight.

In fact one can show that to define $\mathfrak{m}_{\kappa}(F, \lambda)$ it is necessary to glue this cylindrical ends for every boundary component of $F$ that is not close to the lamination. On the other hand, if $\lambda$ contains a leaf $l$ spiraling around a boundary curve, it is clear that it is possible to define the analogous of $\mathfrak{m}_{\kappa}(A)$ for this leaf (that now is the product of a infinite band of width equal to the weight of $l$ and the time-interval $I$ ) and apply the grafting procedure. Notice that if $l$ spirals around a boundary component $c$, the corresponding end on the slice $S \times\{t\}$ in $\mathfrak{m}_{\kappa}(F, \lambda)$ appears complete (in fact a path entering the ends meets the band infinite times so its length cannot be bounded).

From this discussion it appears clear that in this context it is more convenient to use the notion of geodesic lamination on a surface given in Remark 3.12. That is we require that the boundary components of $F$ are contained in the lamination and that paths arriving on the boundary have infinite total mass. In particular for each boundary component either a leaf spirals around it or it carries an infinite weight. With this definition the 0 lamination on $F$ is obtained by putting the weight $+\infty$ on each boundary component.

4.3. Wick rotation - rescaling set up. Let us go back to the statement concerning the canonical correlations. We will see that every spacetime $\mathfrak{m}_{\kappa}(F, \lambda)$ has (rather tame) cosmological time, so that the geometry of the initial singularity will quite naturally arise. The above mentioned Wick rotation-rescaling (possibly only defined on suitable "slabs" of the spacetimes) will be directed by the gradient of the cosmological times. The rescaling functions will be universal in the sense that their values only depend on the cosmological time values: for every $y$ in the domain of definition, $\beta(y)=\beta(\tau(y))$, $\alpha(y)=\alpha(\tau(y))$. We stress that they do not depend on $(F, \lambda)$.

We are going to outline the linked auguments establishing both the constructions of the maps $\mathfrak{m}_{*}$, the geometric correlations and the fact that the materialization maps induce bijections.

Let $(F, \lambda) \in \mathcal{M L}(S)$. With the notations of Section 3, we have

$$
F \subset F^{\mathcal{C}} \subset \hat{F}=\mathbb{H}^{2} / \Gamma .
$$

$\left(F^{\mathcal{C}}, \lambda\right)$ lifts to a $\Gamma$-invariant couple $(H, \tilde{\lambda})$ where $H$ is a straight convex set in $\mathbb{H}^{2}$ equipped with the measured geodesic lamination $\tilde{\lambda}$. The universal covering map

$$
\mathbb{H}^{2} \rightarrow \hat{F}
$$

restricts to the universal covering maps $H \rightarrow F^{\mathcal{C}}, \stackrel{\circ}{H} \rightarrow F$, where $\stackrel{\circ}{H}$ is the interior of $H$. To simplify the notations we make the abuse of always writing $\lambda$ instead of either $\tilde{\lambda},(F, \lambda)$ or $(\stackrel{\leftrightarrow}{H}, \tilde{\lambda})$, that is we will understand $F$ or $\stackrel{\circ}{H}$.

The projective surface

$$
S_{\mathcal{P}}^{\lambda}=\mathfrak{m}_{\mathcal{P}}(\lambda)
$$


will be given in terms of a couple $\left(d_{\mathcal{P}}^{\lambda}, h_{\mathcal{P}}^{\lambda}\right)$ of compatible developing map

$$
d_{\mathcal{P}}^{\lambda}: \widetilde{S} \rightarrow S^{2}
$$

and holonomy representation

$$
h_{\mathcal{P}}^{\lambda}: \pi_{1}(S) \rightarrow P S L(2, \mathbb{C}) .
$$

We denote

$$
p_{\mathcal{P}}^{\lambda}: \tilde{S}_{\mathcal{P}}^{\lambda} \rightarrow S_{\mathcal{P}}^{\lambda}
$$

the corresponding local isomorphic universal covering.

Similarly, every spacetime

$$
Y_{\kappa}^{\lambda}=\mathfrak{m}_{\kappa}(\lambda)
$$

will be specified by a compatible couple $\left(d_{\kappa}^{\lambda}, h_{\kappa}^{\lambda}\right)$,

$$
\begin{gathered}
d_{\kappa}^{\lambda}: \widetilde{S} \times \mathbb{R} \rightarrow \mathbb{X}_{\kappa} \\
h_{\kappa}^{\lambda}: \pi_{1}(S) \rightarrow \operatorname{Isom}^{+}\left(\mathbb{X}_{\kappa}\right) .
\end{gathered}
$$

We denote

$$
p_{\kappa}^{\lambda}: \mathcal{U}_{\kappa}^{\lambda} \rightarrow Y_{\kappa}^{\lambda}
$$

the corresponding local isomorphic universal covering.

For simplicity, we will often identify $S$ with $F, \widetilde{S}$ with $\stackrel{\circ}{H}, \pi_{1}(S)$ with $\Gamma$, and so on.

For every $F$ as above, denote $\lambda_{0}$ the measured geodesic lamination just consisting of the $+\infty$ weighted boundary components of $F^{\mathcal{C}}$. Recall that $\lambda_{0}$ is the initial end-point of any ray in $\mathcal{M L}(F)$. We will describe explicitely the corresponding surface $S_{\mathcal{P}}^{0}$ and spacetimes $Y_{\kappa}^{0}, \mathcal{U}_{\kappa}^{0}$. Every $\lambda \in \mathcal{M L}(F)$ somehow encodes the instructions in order to deform $\left(d_{*}^{0}, h_{*}^{0}\right)$ towards $\left(d_{*}^{\lambda}, h_{*}^{\lambda}\right)$ as it has been make explicit in the case of finite laminations.

4.4. Flat spacetimes classification. Take the hyperboloid model $\mathbb{H}^{2} \subset \mathbb{X}_{0}$ of the hyperbolic plane. The chronological future of 0 in $\mathbb{X}_{0}$ is the cone $I^{+}(0)=\left\{-x_{0}^{2}+x_{1}^{2}+\right.$ $\left.x_{2}^{2}<0, x_{2}>0\right\}$ from 0 over $\mathbb{H}^{2}$. $I^{+}(0)$ has cosmological time $\tau=\left(x_{0}^{2}-\left(x_{1}^{2}+x_{2}^{2}\right)\right)^{1 / 2}$, so that $\mathbb{H}^{2}=\{\tau=1\}$ and 0 is the initial singularity. The future $I^{+}(r)=\left\{-x_{0}^{2}-\right.$ $\left.x_{2}^{2}<0, x_{2}>0\right\}$ of the spacelike geodesic $r=\left\{x_{0}=x_{2}=0\right\}$ has cosmological time $\tau=\left(x_{0}^{2}-x_{2}^{2}\right)^{1 / 2} ; r$ is the initial singularity.

Construction of $\mathcal{U}_{0}^{0}$. The cone $C_{0} H$ from 0 over $H \subset \mathbb{H}^{2}$ is contained in $I^{+}(0)$. The boundary of $C_{0} H$ is made by the cone over the boundary of $H$. Each component of $\partial C_{0} H$, corresponding to a geodesic line $\gamma \subset \partial H$, is the intersection with $I^{+}(0)$ of a hyperplane $P_{\gamma}$, orthogonal to a determined unitary spacelike vector $v_{\gamma}$, that points out of $C_{0} H$. The developing map $d_{0}^{0}$ is an embedding onto the convex domain $\mathcal{U}_{0}^{0}$ of $\mathbb{X}_{0}$ made by the union of $C_{0} H$ with the future of all the rays of the form $\left\{t v_{\gamma}+x \mid t \geq 0\right\}$.

A convenient description of the domain $\mathcal{U}_{0}^{0}$ is as the intersection of half-planes. In fact we have

$$
\mathcal{U}_{0}^{0}=\bigcap_{x \in H} \mathrm{I}^{+}\left(x^{\perp}\right)
$$

This shows that $\mathcal{U}_{0}^{0}$ is convex and future complete. 
Up to isometry of $\mathbb{X}_{0}$, the local model for $\mathcal{U}_{0}^{0}$ at each component of $\partial C_{0}(H)$, is the future $I^{+}\left(r_{+}\right)$of the ray $\left\{x_{1} \geq 0\right\} \subset r$, that is

$$
I^{+}\left(r_{+}\right)=\left(I^{+}(0) \cap\left\{x_{1} \leq 0\right\}\right) \cup\left(I^{+}(r) \cap\left\{x_{1} \geq 0\right\} .\right.
$$

The above cosmological times match at the intersection, producing the cosmological time of the union, that turns to be a $\mathrm{C}^{1}$ function. The ray $r_{+}$is the initial singularity. Then $\mathcal{U}_{0}^{0}$ has cosmological time that coincides with the one of $I^{+}(0)$ on $C_{0} H$; the initial singularity is the spacelike tree made by one vertex at 0 and the rays $t v_{\gamma}, t \geq 0$, emanating from the origin. The action of $\pi_{1}(S)$ on $H$ naturally extends to the whole of $\mathcal{U}_{0}^{0}$, giving the holonomy $h_{0}^{0}$.

Construction of $\mathcal{U}_{0}^{\lambda}$. Let us consider now an arbitrary lamination $\lambda=(L, \mu) \in$ $\mathcal{M L}(F)$. The developing map $d_{0}^{\lambda}$ will be always an embedding onto a convex domain $\mathcal{U}_{0}^{\lambda}$ in $\mathbb{X}_{0}$, obtained as follows. Fix a base-point $x_{0} \in \stackrel{\circ}{H}$ not belonging to the weighted part $L_{W}$ of $\lambda$. For every $x \in \stackrel{\circ}{H} \backslash L_{W}$ choose an arc $c$ transverse to $\lambda$ with end-points $x_{0}$ and $x$. For $t \in c \cap L$, let $v(t) \in \mathbb{R}^{3}$ denote the unitary spacelike vector tangent to $\mathbb{H}^{2}$ at $t$, orthogonal to the leaf through $t$ and pointing towards $x$. For $t \in c \backslash L$, let us set $v(t)=0$. In this way we define a function

$$
v: c \rightarrow \mathbb{R}^{3}
$$

that is continuous on the support of $\mu$. We can define

$$
s(x)=\int_{c} v(t) \mathrm{d} \mu(t) .
$$

It is not hard to see that $s$ does not depend on the path $c$. Moreover, it is constant on every stratum of the stratification determined by $\lambda$, and it is a continuous function on $\mathrm{H} \backslash L_{W}$.

The domain $\mathcal{U}_{0}^{\lambda}$ can be defined in the following way

$$
\mathcal{U}_{0}^{\lambda}=\bigcap_{x \in \mathrm{H} \backslash L_{W}} \mathrm{I}^{+}\left(s(x)+x^{\perp}\right)
$$

where $x^{\perp}$ denote the orthogonal 2-plane to $x$ in $\mathbb{X}_{0}$. Note that this definition is compatible with the one already given for $\mathcal{U}_{0}^{0}$.

The holonomy of $Y_{0}^{\lambda}$ can be defined in this way:

$$
h_{0}^{\lambda}(\gamma)=h_{0}^{0}(\gamma)+\tau(\gamma)
$$

where $h_{0}^{0}: \pi_{1}(S) \rightarrow S O(2,1)$ is the hyperbolic holonomy of $F$ and $\tau(\gamma)$ is the translation by the vector $s\left(\gamma x_{0}\right)$. Since the lamination $\lambda$ is $h$-invariant (being the pull-back of a lamination on $F$ ) the domain $\mathcal{U}_{0}^{\lambda}$ turns to be $h_{0}^{\lambda}$-invariant and $Y_{0}^{\lambda}$ is the quotient of $\mathcal{U}_{0}^{\lambda}$ by this action.

Let us summarize the main properties of this constructions (see [10, 6] for all details).

Theorem 4.8. (1) $\mathcal{U}_{0}^{\lambda}$ coincides with the intersection of the future of its null support planes. In particular it is future complete.

(2) $\mathcal{U}_{0}^{\lambda}$ has $C^{1}$ cosmological time $T_{0}^{\lambda}$ with range $(0,+\infty)$. Every level surface $\mathcal{U}_{0}^{\lambda}(a)=$ $\left(T_{0}^{\lambda}\right)^{-1}(a)$ is a complete Cauchy surface. For every $y \in \mathcal{U}_{0}^{\lambda}$, there is a unique past timelike geodesic segment emanating from $y$ that realizes $T_{0}^{\lambda}(y)$. The union of the past 
end-points of such segments makes the initial singularity $\Sigma_{0}^{\lambda}$. This is a spacelike $\mathbb{R}$-tree injectively immersed in $\mathbb{X}_{0}$.

(3) The action of $\pi_{1}(S)$ on $H$ induces a natural flat spacetime holonomy action on $\mathcal{U}_{0}^{\lambda} \cup \Sigma_{0}^{\lambda}$. The cosmological time descends to the quotient spacetime $Y_{0}^{\lambda}$.

It is convenient to give a general definition of convex subset of $\mathbb{X}_{0}$ satisfying statement (1) in this theorem.

Definition 4.9. A regular domain is an open convex subset of $\mathbb{X}_{0}$ that coincides with the intersection of the future of its null support planes and admits at least two non-parallel null support planes.

Hence we have a well defined map

$$
\mathfrak{m}_{0}: \mathcal{M L}(S) \rightarrow \mathcal{M G H}_{0}(S)
$$

The spacetimes $\mathcal{U}_{0}^{\lambda}$ (and $Y_{0}^{\lambda}$ ) are particularly simple to figure out when $\lambda$ is a finite lamination. In such a case, the local model consists of the future, say $\mathcal{U}_{0}$, of a segment $I=\left[0, \alpha_{0} v_{0}\right]$, where $v_{0}$ is a unitary spacelike vector and $0<\alpha_{0}<\pi$. Here local model means that there is a neighbourhood of each point $p \in Y_{0}^{\lambda}$ that embeds in $\mathcal{U}_{0}$ via an isometry that preserves the cosmological time.

The cosmological time on $\mathcal{U}_{0}$ is realized by geodesics with starting point on $\left[0, \alpha_{0} v\right]$, so there is a natural projection say $r: \mathcal{U}_{0} \rightarrow\left[0, \alpha_{0} v\right]$ sending $p$ to the point on the segment that relaizes the cosmological time.

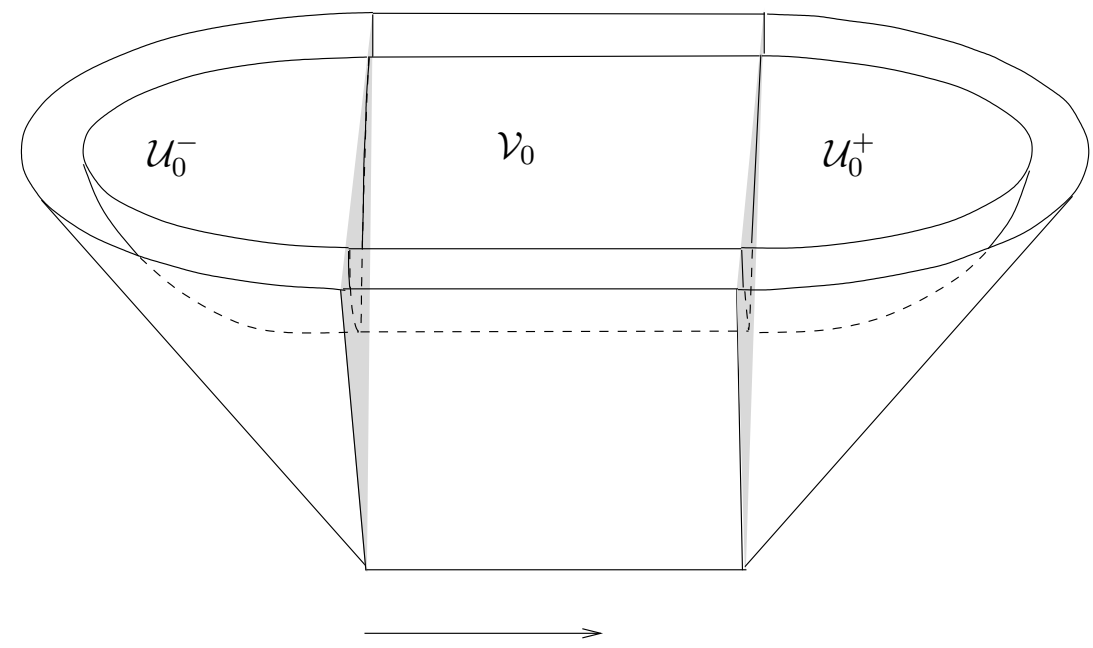

$v_{0}$

Figure 2. The domain $\mathcal{U}_{0}$, its decomposition, and a level surface.

We have a decomposition of $\mathcal{U}_{0}$ in three pieces $\mathcal{U}_{0}^{-}, \mathcal{U}_{0}^{+}, \mathcal{V}$ defined in the following way:

$$
\begin{aligned}
& \mathcal{U}_{0}^{-}=r^{-1}(0) ; \\
& \mathcal{V}=r^{-1}\left(0, \alpha_{0} v_{0}\right) \\
& \mathcal{U}_{0}^{+}=r^{-1}\left(\alpha_{0} v_{0}\right)
\end{aligned}
$$


We denote by $\mathcal{U}_{0}^{+}(a), \mathcal{U}_{0}^{-}(a), \mathcal{V}(a)$ the intersections of corresponding domains with the surface $\mathcal{U}_{0}(a)$. Surfaces $\mathcal{U}_{0}^{+}(a)$ are hyperbolic of constant curvature $-1 / a^{2}$. On the other hand, the parametrization of $\mathcal{V}$ given by

$$
\left(0, \alpha_{0}\right) \times l_{0} \ni(t, y) \mapsto a y+t v_{0} \in \mathcal{V}(a)
$$

produces two orthogonal geodesic foliations on $\mathcal{V}$. The parametrization restricted to horizontal leaves is an isometry, whereas on the on vertical leaves it acts as a rescaling of factor $a$. Thus $\mathcal{V}(a)$ is a Euclidean band of width $\alpha_{0}$. Note that by formally setting $\alpha_{0}=+\infty$, and removing $\mathcal{U}_{0}^{+}$, we recover the above local model for $\mathcal{U}_{0}^{0}$ at each component of $\partial C_{0}(H)$.

The initial singularity of a flat spacetime corresponding to a finite lamination is a simplicial metric tree. On the other hand, in [10] we prove also a suitable continuous dependence of $\mathcal{U}_{0}^{\lambda}$ on $\lambda$. By using the density of finite laminations, this implies that spacetimes corresponding to finite laminations provide us with good approximations of arbitrary ones.

Asymptotic states. In general, the cosmological time level surface $\mathcal{U}_{0}^{\lambda}(1)\left(Y_{0}^{\lambda}(1)\right)$ is a $\mathrm{C}^{1}$ spacelike surface; with the induced Riemannian metric it realizes the grafting of $\stackrel{\circ}{H}$ (the hyperbolic surface $F$ ) at the measured geodesic lamination $\lambda$. By taking the rescaled level surface $(1 / a) Y_{0}^{\lambda}(a)$, we get a 1-parameter family of grafting of $F$. More precisely we get that $(1 / a) Y_{0}^{\lambda}(a)$ is obtained by grafting $F$ along $\lambda / a$.

When $a \rightarrow+\infty$ the geometries of $(1 / a) Y_{0}^{\lambda}(a)$ converge to $F$. The geometry of the initial singularity $\Sigma_{0}^{\lambda}$ of $\mathcal{U}_{0}^{\lambda}$, together with the isometric action of $\Gamma$ on it, is "dual" to the geometry of the measured lamination $\lambda$, and can be recovered by means of the asymptotic behaviour of the level surfaces $\mathcal{U}_{0}^{\lambda}(a)$ (equipped with the respective isometric actions of $\Gamma$ on them), when $a \rightarrow 0$.

The inverse map of $\mathfrak{m}_{0}$. The image of $\mathfrak{m}_{0}$ consists of spacetimes whose universal covering is a regular domain that is, in particular, future complete. On the other hand, general results due to Barbot [6] on flat spacetimes, applied in our finite type situation, imply that, possibly reversing the time orientation, every spacetime $Y$ in $\mathcal{M G \mathcal { H }}_{0}(S)$ is future complete, and its universal covering is a regular domain $\mathcal{U} \neq I^{+}(r)$. So it is natural to consider the quotient $\mathcal{M G \mathcal { H }}_{0}(S) / \pm$, up to time orientation reversing. We are going to outline the steps leading to the inverse map of $\mathfrak{m}_{0}$, defined on it. First one shows that every regular domain $\mathcal{U}$ has cosmological time $T$ that satisfies point (2) of Theorem 4.4. We consider the level surface $\mathcal{U}(1)$. We have a natural continuous retraction

$$
r: \mathcal{U}(1) \rightarrow \Sigma_{\mathcal{U}}
$$

onto the initial singularity. Moreover, the gradient of $T$ is a unitary vector field, hence it induces the Gauss map

$$
N: \mathcal{U}(1) \rightarrow \mathbb{H}^{2}
$$

The closure $H_{\mathcal{U}}$ of the image of $N$ in $\mathbb{H}^{2}$ is a straight convex set. If $\mathcal{U} \rightarrow Y$ is a universal covering of $Y \in \mathcal{M G H}_{0}(S)$, the action of $\pi_{1}(S)$ extends to $H_{\mathcal{U}}$, and makes it a universal covering of $F_{\mathcal{U}}^{\mathcal{C}}$, for some $F_{\mathcal{U}} \in \widetilde{\mathcal{T}}(S)$. We take the partition of $\mathcal{U}(1)$ given by the closed sets $r^{-1}(y), y \in \Sigma_{\mathcal{U}}$. Via the retraction, we can pullback to this partition the metric structure of $\Sigma_{\mathcal{U}}$, and (in a suitable sense) we can project everything onto $H_{\mathcal{U}}$, by means 
of the Gauss map. More precisely, if $r^{-1}(y)$ is 1-dimensional, then it is a geodesic line, so that the union of such lines makes a lamination in $\mathcal{U}(1)$. We can define on it a transverse measure such that the mass of any transverse path is given by the integral of the Lorentzian norm of the derivative of $r$. A measured geodesic lamination $\lambda_{\mathcal{U}}$ on $H_{\mathcal{U}}$ is obtained via the push-forward by $N$ of this lamination on $\mathcal{U}(1)$. This descends to a lamination $\lambda_{\mathcal{U}}$ on $F$. So we eventually get $\mathfrak{m}_{0}^{-1}(Y)=\left(F_{\mathcal{U}}, \lambda_{\mathcal{U}}\right)$. This achieves our classification of flat $M G H$ spacetimes of finite type.

4.5. Wick rotation: flat Lorentzian vs hyperbolic geometry. Although we adopt a slightly different definition of the involved measured geodesic laminations, the bijective map

$$
\mathfrak{m}_{\mathcal{P}}: \mathcal{M L}(S) \rightarrow \mathcal{P}(S)
$$

is due to Kulkarni-Pinkall [37] and extends one due to Thurston for compact $S$. This is unfolded in terms of a 3-dimensional hyperbolic construction. We are going to describe it, by performing at the same time the canonical Wick rotation establishing the correlation between the flat spacetimes $Y \in \mathcal{M G \mathcal { H }}_{0}(S)$ and suitable hyperbolic 3-manifolds.

For every $Y \in \mathcal{M G H}_{0}(S)\left(Y=Y_{0}^{\lambda}\right)$, with universal covering $\mathcal{U} \rightarrow Y$, and cosmological time $T$, we construct a local $\mathrm{C}^{1}$-diffeomorphism

$$
d_{\mathbb{H}}: \mathcal{U}(>1) \rightarrow \mathbb{H}^{3}
$$

and a compatible holonomy

$$
h_{\mathbb{H}}: \pi_{1}(S) \rightarrow P S L(2, \mathbb{C})
$$

realizing a (non complete) hyperbolic structure $M=M_{Y}$ on $Y(>1)$. This verifies the following properties:

(1) The hyperbolic metric of $M$ is obtained by the Wick rotation of the flat Lorentzian metric on $Y(>1)$, directed by the gradient of $T$, with universal rescaling functions:

$$
\alpha=\frac{1}{T^{2}-1}, \quad \beta=\frac{1}{\left(T^{2}-1\right)^{2}} .
$$

(2) Recall that the closure $H$ of the Gauss map image is the straight convex set realizing the future asymptotic geometry of $\mathcal{U}$. Then the map $d_{\mathbb{H}}$ extends (in $h_{\mathbb{H}}$ equivariant way) to

$$
d_{\mathbb{H}}: \mathcal{U}(\geq 1) \cup H \rightarrow \mathbb{H}^{3}
$$

such that:

(a) The restriction of $d_{\mathbb{H}}$ to $\mathcal{U}(>1) \cup H$ corresponds to the completion of the manifold $M$. The restriction to $\stackrel{\circ}{H}$ is a locally isometric pleated immersion in $\mathbb{H}^{3}$, having the measured geodesic lamination $\lambda$ as bending locus. This gives the so called hyperbolic boundary of $M$. The level surfaces $\mathcal{U}(a), a>1$, correspond via $d_{\mathbb{H}}$ to level surfaces of the distance function $\Delta$ on $M$ from its hyperbolic boundary, so that the inverse Wick rotation is directed by the gradient of $\Delta$. More precisely the following formula holds:

$$
\Delta=\operatorname{arctgh}(1 / T) \text {. }
$$


(b) The restriction $\left.d_{\mathbb{H}}\right|_{\mathcal{U}(1)}$ actually coincides with the developing map of the complex projective structure $S_{\mathcal{P}}^{\lambda}$

$$
d_{\mathcal{P}}: \mathcal{U}(1) \rightarrow S^{2}
$$

so that

$$
h_{\mathcal{P}}=h_{\mathbb{H}} .
$$

The spacelike metric of $\mathcal{U}(1)(Y(1))$ coincides with the Thurston metric of this projective surface, as well as its canonical stratification coincides with the stratification induced by the retraction $r$ of $\mathcal{U}(1)$ onto the initial singularity. This gives the so called asymptotic complex projective boundary of $M$. In fact $M$ turns to be the $H$-hull of $Y(1)$.

Remark 4.10. We recall here the definition of the above mentioned "Thurston metric" and "canonical stratification". Let us take a complex projective structure on our surface $S$ and consider a developing map

$$
D: \tilde{S} \rightarrow S^{2}
$$

Pulling back the standard unitary-sphere metric of $S^{2}$ on $\tilde{S}$ is not a well-defined operation, as it depends on the choice of the developing map. Nevertheless, by the compactness of $S^{2}$, the completion $\bar{S}$ of $\tilde{S}$ with respect to such a metric is well-defined. It turns out that in our finite-type situation, $\bar{S} \backslash \tilde{S}$ contains at least 2 points (we say that it is of hyperbolic type). A round disk in $\tilde{S}$ is a set $\Delta$ such that $\left.D\right|_{\Delta}$ is injective and the image of $\Delta$ is a round disk in $S^{2}$ (this notion is well defined because $P S L(2, \mathbb{C}$ ) sends round disks onto round disks). Given a maximal disk $\Delta$ (with respect to the inclusion), we can consider its closure $\bar{\Delta}$ in $\bar{S}$.

$\bar{\Delta}$ is sent by $D$ to the closed disk $\overline{D(\Delta)}$. In particular, if $g_{\Delta}$ denotes the pull-back on $\Delta$ of the standard hyperbolic metric on $D(\Delta)$, we can consider the boundary of $\Delta$ in $\hat{S}$ as its ideal boundary. Since $\Delta$ is maximal, $\bar{\Delta}$ is not contained in $\tilde{S}$. So, if $\Lambda_{\Delta}$ denotes the set of points in $\bar{\Delta} \backslash \tilde{S}$, let $\hat{\Delta}$ be the convex hull in $\left(\Delta, g_{\Delta}\right)$ of $\Lambda_{\Delta}$ (by maximality $\Lambda_{\Delta}$ contains at least two points). In [37] it is proved that for every point $p \in \tilde{S}$, there exists a unique maximal disk $\Delta$ containing $p$ such that $p \in \hat{\Delta}$. So, $\{\hat{\Delta} \mid \Delta$ is a maximal disk $\}$ is a partition of $\tilde{S}$. We call it the canonical stratification of $\tilde{S}$. Clearly the stratification is invariant under the action of $\pi_{1}(S)$.

Let $g$ be the Riemannian metric on $\tilde{S}$ that coincides at $p$ with the metric $g_{\Delta}$, where $\Delta$ is the maximal disk such that $p \in \hat{\Delta}$. It is a conformal metric, in the sense that it makes $D$ a conformal map. It is $\mathrm{C}^{1,1}$ and is invariant under the action of $\pi_{1}(S)$. So, it induces a metric on $\tilde{S}$. We call it the Thurston metric on $\tilde{S}$.

Finally let us recall the construction of the $H$-hull of $S$. For $p \in \tilde{S}$, let $\Delta(p)$ be the maximal disk such that $p \in \hat{\Delta}$. The image of $\Delta$ via dev is a round disk in $S^{2}$, so its boundary is the trace of a hyperbolic plane $P(p)$ in $\mathbb{H}^{3}$. Let $c_{p}$ the geodesic half-line with an end-point at $\operatorname{dev}(p)$ an end-point on $P(p)$ and orthogonal to $P(p)$. Then the developing map of the $H$-hull of $S$ is the map

$$
\tilde{S} \times(0,+\infty) \ni(p, t) \mapsto c_{p}(t) \in \mathbb{H}^{3} .
$$


Notice that if $S$ is quasi-Fuchsian, the $H$-hull is simply the end of the corresponding quasi-Fuchsian manifold facing $S$.

About the rescaling functions. Before proving the Theorem we want to give some euristhic motivation for formulae of Wick Rotation. The point is that we want to construct a Wick Rotation transforming $Y_{0}^{\lambda}$ (or some slab) into the $H$-hull, say $H$, of $G r_{\lambda}(F)$, in such a way that the CT level surfaces are sent to level surfaces of the distance from the hyperbolic boundary and rescaling functions are constant on level surfaces. Now suppose that such a Wick Rotation exists. Let $\Delta(T)$ be such a way that the Wick Rotation transforms $Y(T)$ into $M(\Delta(T))$, and let $\alpha(T)$ and $\beta(T)$ be the horizontal and vertical rescaling functions.

By formulae (2) and (41) we should have

$$
(\alpha(T))^{1 / 2} T G r_{\lambda / T}(F)=\operatorname{ch} \Delta(T) G r_{\operatorname{tgh} \Delta(T) \lambda}
$$

Since $G r_{t \lambda}(F)$ is conformally equivalent to $G r_{s \lambda}(F)$ iff $s=t$ we deduce that

$$
T=1 / \operatorname{tgh}(\Delta(T))
$$

that is $\Delta(T)=\operatorname{arctgh}(1 / T)$. Moreover we have

$$
\alpha(T)=\operatorname{ch}^{2}(\Delta(T)) / T^{2}=1 /\left(T^{2}-1\right) .
$$

Finally, let $X$ denote the gradient of $T$ with respect to the flat metric and $Y$ denote the gradient of $\Delta$ with respect to the hyperbolic metric. We have $X=-\beta^{1 / 2} Y$. On the other hand $\langle X, Y\rangle_{H y p}=d \Delta(X)=\Delta^{\prime}(T) d T(X)=-1 /\left(T^{2}-1\right)$. Thus $\beta(T)=$ $1 /\left(T^{2}-1\right)^{2}$.

Summarizing, if some Wick Rotation exists satysfying properties we have required, then necessarily $\alpha=1 /\left(T^{2}-1\right)$ and $\beta=1 /\left(T^{2}-1\right)^{2}$.

Bending cocycle A key step in the construction is the bending of $\stackrel{\circ}{H}$ in $\mathbb{H}^{3}$ along a measured geodesic lamination $\lambda$. We mostly refer to the Epstein-Marden paper [24] where this hyperbolic bending has been carefully studied (in the case of $\stackrel{\circ}{H}=\mathbb{H}^{2}$; however the constructions extend straightforwardly to the general case). In fact in [24] one considers quake-bend maps, more generally associated to complex-valued transverse measures on a lamination $\mathcal{L}$. Bending maps correspond to imaginary valued measures. So, given a measured geodesic lamination $\lambda=(\mathcal{L}, \mu) \in \mathcal{M L}(F)$, we take $i \mu$ in order to get the corresponding bending map.

The bending cocycles. We fix once and for all an embedding of $\mathbb{H}^{2}$ into $\mathbb{H}^{3}$ as a totally geodesic hyperbolic plane.

Given $\lambda$ on $H$ as usual, we define first the associated bending cocycle (recall a similar notion already introduced in Section 3 relatively to the earthquakes). This is a map

$$
B_{\lambda}: \stackrel{\circ}{H} \times \stackrel{\circ}{H} \rightarrow P S L(2, \mathbb{C})
$$

which satisfies the following properties:

(1) $B_{\lambda}(x, y) \circ B_{\lambda}(y, z)=B_{\lambda}(x, z)$ for every $x, y, z \in \stackrel{\circ}{H}$.

(2) $B_{\lambda}(x, x)=I d$ for every $x \in \stackrel{\circ}{H}$.

(3) $B_{\lambda}$ is constant on the strata of the stratification of $\stackrel{\circ}{H}$ determined by $\lambda$.

(4) If $\lambda_{n} \rightarrow \lambda$ on a $\epsilon$-neighbourhood of the segment $[x, y]$ and $x, y \notin L_{W}$, then $B_{\lambda_{n}}(x, y) \rightarrow B_{\lambda}(x, y)$. 
If $\lambda$ is finite, then there is an easy description of $B_{\lambda}$. If $l$ is an oriented geodesic of $\mathbb{H}^{3}$, let $X_{l} \in \mathfrak{s l}(2, \mathbb{C})$ denote the infinitesimal generator of the positive rotation around $l$ such that $\exp \left(2 \pi X_{l}\right)=I d$ (since $l$ is oriented the notion of positive rotation is well defined). Now take $x, y \in \stackrel{\circ}{H}$. If they lie in the same leaf of $\lambda$ then put $B_{\lambda}(x, y)=I d$. If both $x$ and $y$ do not lie on the support of $\lambda$, then let $l_{1}, \ldots, l_{s}$ be the geodesics of $\lambda$ meeting the segment $[x, y]$ and $a_{1}, \ldots, a_{s}$ be the respective weights. Let us consider the orientation on $l_{i}$ induced by the half plane bounded by $l_{i}$ containing $x$ and non-containing $y$. Then put

$$
B_{\lambda}(x, y)=\exp \left(a_{1} X_{1}\right) \circ \exp \left(a_{2} X_{2}\right) \circ \cdots \circ \exp \left(a_{s} X_{s}\right) .
$$

If $x$ lies in $l_{1}$ use the same construction, but replace $a_{1}$ by $a_{1} / 2$; if $y$ lies in $l_{s}$ replace $a_{s}$ by $a_{s} / 2$.

The bending cocycle is not continuous on the whole definition set. However, there is a natural continuous "pull-back" of it to a cocycle defined on the flat spacetime $\mathcal{U}=\mathcal{U}_{0}^{\lambda}$

$$
\hat{B}_{\lambda}: \mathcal{U} \times \mathcal{U} \rightarrow P S L(2, \mathbb{C})
$$

such that

$$
\hat{B}_{\lambda}(p, q)=B_{\lambda}(N(p), N(q))
$$

for $p, q$ such that $N(p)$ and $N(q)$ do not lie on $L_{W}$.

This map is locally Lipschitz (with respect to the Euclidean distance on $\mathcal{U}$ ). Moreover, for every compact set $K$ of $\mathcal{U}$, the Lipschitz constant on $K \times K$ depends only on $N(K)$, on the diameter of $r(1, \cdot)(K)$ and on the maximum $M$ and minimum $m$ of $T$ on $K$.

The bending map. Fix a base point $x_{0}$ of $\stackrel{\circ}{H}\left(x_{0}\right.$ is supposed not to be in $\left.L_{W}\right)$. The bending map of $\stackrel{\circ}{H}$ along $\lambda$ is

$$
F=F_{\lambda}: \stackrel{\circ}{H} \ni x \mapsto B\left(x_{0}, x\right) x \in \mathbb{H}^{3} .
$$

$F$ satisfies the following properties:

(1) It does not depend on $x_{0}$ up to post-composition of elements of $P S L(2, \mathbb{C})$.

(2) It is a 1-Lipschitz map.

(3) If $\lambda_{n} \rightarrow \lambda$ then $F_{\lambda_{n}} \rightarrow F_{\lambda}$ with respect to the compact open topology.

The Wick rotation. We are ready to construct the local $\mathrm{C}^{1}$-diffeomorphism

$$
d_{\mathbb{H}}: \mathcal{U}(>1) \rightarrow \mathbb{H}^{3}
$$

with the properties outlined at the beginning of this Section.

Recall the continuous cocycle $\hat{B}=\hat{B}_{\lambda}$ defined above on the whole of $\mathcal{U} \times \mathcal{U}$. Since both $\mathbb{H}^{3}$ and $\mathbb{H}^{2} \subset \mathbb{H}^{3}$ are oriented, the normal bundle is oriented too. Let $v$ denote the normal vector field on $\mathbb{H}^{2}$ that is positive oriented with respect to the orientation of the normal bundle. Let us take $p_{0} \in N^{-1}\left(x_{0}\right)$ and for $p \in \mathcal{U}(>1)$ consider the geodesic ray $c_{p}$ of $\mathbb{H}^{3}$ starting from $F(N(p))$ with speed vector equal to $w(p)=\hat{B}\left(p_{0}, p\right)_{*}(v(N(p)))$. Thus $d_{\mathbb{H}}$ is defined in the following way:

$$
d_{\mathbb{H}}(p)=c_{p}(\operatorname{arctgh}(1 / T(p)))=\exp _{F(N(p))}\left(\operatorname{arctgh}\left(\frac{1}{T(p)}\right) w(p)\right) .
$$

As usual, we make everything explicit on the local models of $\mathcal{U}_{0}^{0}$ and of flat spacetimes associated to finite laminations. 
Local model of the Wick rotation for finite laminations. Consider as above the future $\mathcal{U}_{0}$ of a spacelike segment in $\mathbb{X}_{0}$ (adopting the same notations). We introduce suitable $\mathrm{C}^{1,1}$ coordinates on $\mathcal{U}_{0}$. Denote by $l_{a}$ the boundary of $\mathcal{U}_{0}^{-}(a)$ and by $d_{a}$ the intrinsic distance of $\mathcal{U}_{0}(a)$. Fix a point $z_{0}$ on $l_{0}$ and denote by $\hat{z}_{a} \in l_{a}$ the point such that $N\left(\hat{z}_{a}\right)=z_{0}$.

For every $x \in \mathcal{U}_{0}(a)$ there is a unique point $\pi(x) \in l_{a}$ such that $d_{a}\left(x, l_{a}\right)=d_{a}(x, \pi(x))$. Then we consider coordinates $T, \zeta, u$, where $T$ is again the cosmological time, and $\zeta, u$ are defined in the following way

$$
\begin{aligned}
& \zeta(x)=\epsilon(x) d_{T(x)}\left(x, l_{T(x)}\right) / T(x) \\
& u(x)=\epsilon^{\prime}(x) d_{T(x)}\left(\pi(x), \hat{z}_{T(x)}\right) / T(x)
\end{aligned}
$$

where $\epsilon(x)$ (resp. $\left.\epsilon^{\prime}(x)\right)$ is -1 if $x \in \mathcal{U}_{0}^{-}$(resp. $\pi(x)$ is on the left of $\hat{z}_{T(x)}$ ) and is 1 otherwise.

Choose coordinates $\left(y_{0}, y_{1}, y_{2}\right)$ of the Minkowski space such that $v_{0}=(0,0,1)$ and $z_{0}=(1,0,0)$. Thus the parametrization induced by $T, \zeta, u$ is

$$
(T, u, \zeta) \mapsto \begin{cases}T(\operatorname{ch} u \operatorname{ch} \zeta, \operatorname{sh} u \operatorname{ch} \zeta, \operatorname{sh} \zeta) & \text { if } \zeta<0 \\ T(\operatorname{ch} u, \operatorname{sh} u, \zeta) & \text { if } \zeta \in\left[0, \alpha_{0} / T\right] \\ T\left(\operatorname{ch} u \operatorname{ch} \zeta^{\prime}, \operatorname{sh} u \operatorname{ch} \zeta^{\prime}, \operatorname{sh} \zeta^{\prime}+\alpha_{0} / T\right) & \text { otherwise }\end{cases}
$$

where we have put $\zeta^{\prime}=\zeta-\alpha_{0} / T$.

With respect to these coordinates the metric take the following form:

$$
h_{0}(T, \zeta, u)= \begin{cases}-\mathrm{d} T^{2}+T^{2}\left(\mathrm{~d} \zeta^{2}+\mathrm{ch}^{2} \zeta \mathrm{d} u^{2}\right) & \text { if } \zeta<0, \\ -\mathrm{d} T^{2}+T^{2}\left(\mathrm{~d} \zeta^{2}+\mathrm{d} u^{2}\right) & \text { if } \zeta \in\left[0, \alpha_{0} / T\right] \\ -d T^{2}+T^{2}\left(\mathrm{~d} \zeta^{2}+\operatorname{ch}^{2}\left(\zeta^{\prime}\right) \mathrm{d} u^{2}\right) & \text { otherwise. }\end{cases}
$$

Notice that the gradient of $T$ is just the coordinate field $\frac{\partial}{\partial T}$.

The Gauss map takes the following form

$$
N(T, \zeta, u)= \begin{cases}(\operatorname{ch} u \operatorname{ch} \zeta, \operatorname{sh} u \operatorname{ch} \zeta, \operatorname{sh} \zeta) & \text { if } \zeta<0 \\ (\operatorname{ch} u, \operatorname{sh} u, 0) & \text { if } \zeta \in\left[0, \alpha_{0} / T\right] \\ \left(\operatorname{ch} u \operatorname{ch} \zeta^{\prime}, \operatorname{sh} u \operatorname{ch} \zeta^{\prime}, \operatorname{sh} \zeta^{\prime}\right) & \text { otherwise }\end{cases}
$$

and the bending cocycle $\hat{B}_{0}\left(p_{0},(T, \zeta, u)\right)$ is the rotation around $l_{0}$ of angle equal to 0 if $\zeta<0, \zeta$ if $\zeta \in\left[0, \alpha_{0} / T\right], \alpha_{0} / T$ otherwise.

Let $\mathbb{H}^{3}$ be identified with the set of timelike unit vectors in the $3+1$-Minkowski space $\mathbb{M}^{4}$. We can choose affine coordinates on $\mathbb{M}^{4}$ in such a way the inclusion $\mathbb{H}^{3} \subset \mathbb{H}^{4}$ is induced by the inclusion $\mathbb{X}_{0} \rightarrow \mathbb{M}^{4}$ given by $\left(x_{0}, x_{1}, x_{2}\right) \mapsto\left(x_{0}, x_{1}, x_{2}, 0\right)$. Thus the general rotation around $l_{0}$ of angle $\alpha$ is represented by the linear transformation $T_{\alpha}$, such that

$$
T_{\alpha}\left(e_{0}\right)=e_{0}, T_{\alpha}\left(e_{1}\right)=e_{1}, T\left(e_{2}\right)=\cos \alpha e_{2}+\sin \alpha e_{3}, T_{\alpha}\left(e_{3}\right)=-\sin \alpha e_{2}+\cos \alpha e_{3}
$$




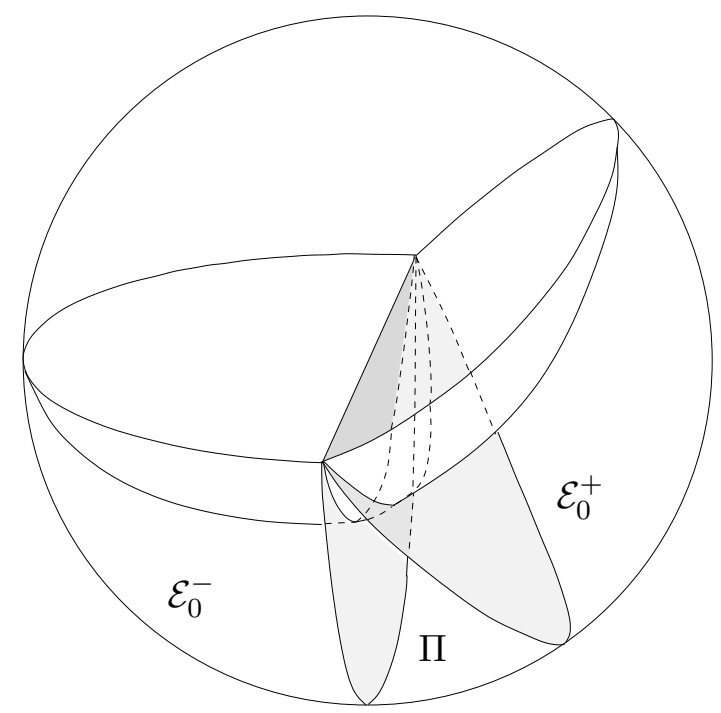

Figure 3. The image $\mathcal{E}_{0}$ of $D_{0}$ and its decomposition.

where $\left(e_{0}, e_{1}, e_{2}, e_{3}\right)$ is the canonical basis of $\mathbb{R}^{4}$. Thus, we can write in local coordinates $d_{\mathbb{H}}=D_{0}$

$$
D_{0}(T, u, \zeta) \mapsto\left\{\begin{array}{l}
\operatorname{ch} \delta(\operatorname{ch} \zeta \operatorname{ch} u, \operatorname{ch} \zeta \operatorname{sh} u, \operatorname{sh} \zeta, 0)+\operatorname{sh} \delta(0,0,0,1) \\
\text { if } \zeta \leq 0 ; \\
\operatorname{ch} \delta(\operatorname{ch} u, \operatorname{sh} u, 0,0)+\operatorname{sh} \delta\left(0,0,-\sin \frac{\zeta}{\operatorname{tgh} \delta}, \cos \frac{\zeta}{\operatorname{tgh} \delta}\right) \\
\text { if } \zeta \in\left[0, \alpha_{0} / T\right] ; \\
\operatorname{ch} \delta\left(\operatorname{ch} \zeta^{\prime} \operatorname{ch} u, \operatorname{ch} \zeta^{\prime} \operatorname{sh} u, \operatorname{sh} \zeta^{\prime} \cos \alpha_{0}, \operatorname{sh} \zeta^{\prime} \sin \alpha_{0}\right)+ \\
\operatorname{sh} \delta\left(0,0,-\sin \alpha_{0}, \cos \alpha_{0}\right) \\
\text { otherwise }
\end{array}\right.
$$

where $\delta=\operatorname{arctgh}(1 / T)$ and $\zeta^{\prime}=\eta-\alpha_{0} / T$. This map is clearly smooth for $\zeta \neq 0, \alpha_{0} / T$. Since the derivatives of $D_{0}$ with respect the coordinates fields glue along $\zeta=0$ and $\zeta=\alpha_{0} T$ the map $D_{0}$ is $\mathrm{C}^{1}$. It is not hard to see that the derivatives are locally Lipschitz. One can check by direct computation that $D_{0}^{*}(g)$ is obtained by the canonical Wick rotation. The same formulae hold on $\mathcal{U}_{0}^{0}$, providing that we replace $\mathcal{U}_{0}^{+} \cup \mathcal{V}$ by $r^{-1}\left(0,+\infty v_{0}\right)$, the inverse image of the open ray.

The holonomy $h_{\mathbb{H}}$. Recall that $(F, \lambda) \in \mathcal{M L}(S), F \subset F^{\mathcal{C}} \subset \hat{F}=\mathbb{H}^{2} / \Gamma, F=\stackrel{\circ}{H} / \Gamma$. Then (see [24]) the bending cocycle satisfies:

$$
B_{\lambda}(\gamma x, \gamma y)=\gamma \circ B(x, y) \circ \gamma^{-1}
$$

for every $\gamma \in \Gamma$.

Consider a bending map

$$
F_{\lambda}: \stackrel{\circ}{H} \rightarrow \mathbb{H}^{3}
$$

For $\gamma \in \Gamma$ let us define

$$
h_{\mathbb{H}}(\gamma)=B_{\lambda}\left(x_{0}, \gamma x_{0}\right) \circ \gamma \in P S L(2, \mathbb{C})
$$




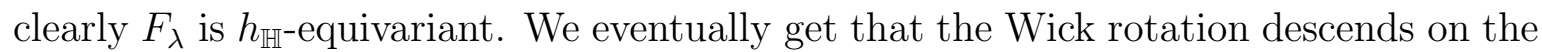
quotient spacetime $Y=Y_{0}^{\lambda}$, this gives the required hyperbolic structure $M$ on $Y(>1)$, having as asymptotic boundary the projective surface $S_{\mathcal{P}}^{\lambda}$.

4.6. Flat vs de Sitter Lorentzian geometry. In order to classify $M G H$ de Sitter spacetimes of finite type in terms of complex projective structures, we widely refer to [45] where the case of compact Cauchy surfaces was treated. In fact we can check that all constructions work as well by simply letting the Cauchy surface be complete of finite type. Let us summarize the main steps of this classification:

(1) We associate to every complex projective structure on a surface of finite type $S$ a so called standard spacetime belonging to $\mathcal{M G H}_{1}(S)$. It turns that it is future complete. By composing with the parametrization $\mathfrak{m}_{\mathcal{P}}: \mathcal{M} \mathcal{L}(S) \rightarrow \mathcal{P}(S)$, we eventually construct the injective map $\mathfrak{m}_{1}: \mathcal{M L}(S) \rightarrow \mathcal{M G H}_{1}(S)$.

(2) We show that, possibly inverting the time orientation, every spacetime in $\mathcal{M G H}_{-1}(S)$ is standard, that is $\mathfrak{m}_{1}: \mathcal{M L}(S) \rightarrow \mathcal{M G H}_{1}(S) / \pm$ is a bijection (with the same meaning of \pm as for $\mathfrak{m}_{0}$ ).

We recall the construction of these standard spacetimes. Given a projective structure on $S$, with developing map

$$
d: \tilde{S} \rightarrow S_{\infty}^{2}
$$

we perform a construction which is dual to the one made for the $H$-hulls. Recall the canonical stratification of $\tilde{S}$. For every $p \in \tilde{S}$ let $U(p)$ denote the stratum passing through $p$ and $U^{*}(p)$ be the maximal ball containing $U(p)$. Now $d\left(U^{*}(p)\right)$ is a ball in $S_{\infty}^{2}$ which determines a hyperbolic plane in $\mathbb{H}^{3}$. Let $\rho(p)$ denote the point in $\mathbb{X}_{1}$ corresponding to this plane: the map $\rho: \tilde{S} \rightarrow \mathbb{X}_{1}$ turns out to be continuous. There exists a unique timelike geodesic $c_{p}$ in $\mathbb{X}_{1}$ joining $\rho(p)$ to $d(p)$ so we can define the map

$$
\hat{d}: \Delta \times(0,+\infty) \ni(p, t) \mapsto c_{p}(t) \in \mathbb{X}_{1}
$$

This map is a developing map for the required standard de Sitter spacetime. A compatible holonomy follows by a natural equivariant version of the construction.

Assume now that the the projective structure is encoded by $(F, \lambda) \in \mathcal{M L}(S)$, via $\mathfrak{m}_{\mathcal{P}}$. We eventually realize that the construction of $\mathfrak{m}_{1}$ can be obtained via a canonical rescaling performed on $Y_{\lambda}^{0}(<1)$. More precisely we realize $\hat{d}$ as a $\mathrm{C}^{1}$ developing map

$$
d_{1}^{\lambda}: \mathcal{U}_{\lambda}(<1) \rightarrow \mathbb{X}_{1}
$$

obtained as a sort of semi-analytic continuation of the hyperbolic developing map $d_{\mathbb{H}}$ constructed in the previous Section, and we have:

Theorem 4.11. The spacetime $\mathcal{U}_{\lambda}^{1}\left(Y_{\lambda}^{1}\right)$, obtained from $\mathcal{U}_{\lambda}^{0}(<1)\left(Y_{\lambda}^{0}(<1)\right.$ ) via the rescaling directed by the gradient of its cosmological time $T$ and with rescaling functions

$$
\alpha=\frac{1}{1-T^{2}} \quad \beta=\frac{1}{\left(1-T^{2}\right)^{2}} .
$$

is the standard de Sitter spacetime corresponding to the projective structure on $\mathcal{U}_{\lambda}^{0}(1)$ $\left(Y_{\lambda}^{0}(1)\right)$ produced by the Wick rotation. 


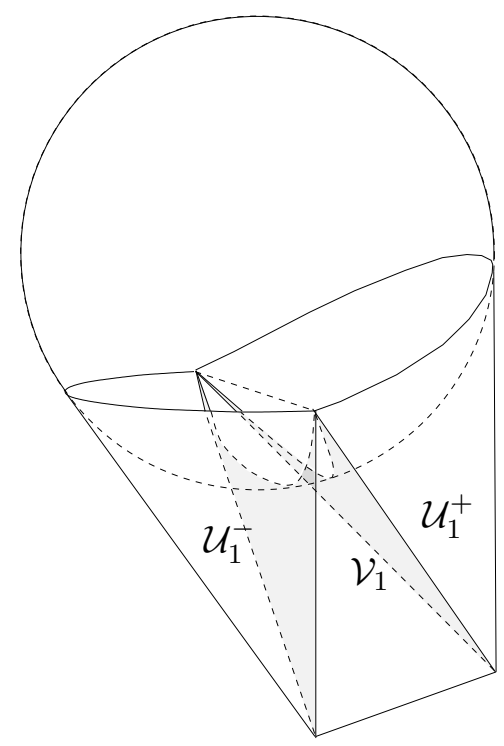

Figure 4. A standard de Sitter spacetime - local model

The construction of $d_{1}^{\lambda}$ is very simple. We regard both $\mathbb{H}^{3}$ and $\mathbb{X}_{1}$ as open sets of the real projective space (Klein models), separated by the quadric $S_{\infty}^{2}$. If $s$ is a geodesic integral line of the gradient of the cosmological time, $s_{>1}=s \cap \mathcal{U}_{\lambda}^{0}(>1)$ is sent by $d_{\mathbb{H}}$ onto a geodesic ray of $\mathbb{H}^{3}$. We define $d_{1}^{\lambda}$ on $s_{<1}$ in such a way that it parameterizes the timelike geodesic ray in $\mathbb{X}_{1}$ contained in the projective line (in the Klein model) determined by $d_{\mathbb{H}}\left(s_{>1}\right)$.

The proof, as well as the explicit computation for our favourite local models (evoked in the Figure) are similar to the ones made for the Wick rotation, so we omit them. A main point in proving that $\mathfrak{m}_{1}$ is a bijection consists in

Proposition 4.12. (1) Every $Y \in \mathcal{M G \mathcal { H }}_{1}(S)$ has $C^{1}$ cosmological time, and every level surface is a complete Cauchy surface.

(2) If $Y=Y_{\lambda}^{1}$ with universal covering $\mathcal{U}_{\lambda}^{1}$, then the cosmological time of $\mathcal{U}_{\lambda}^{1}$ is

$$
\tau=\operatorname{arctgh}(T) \text {. }
$$

$T$ being the cosmological time of $\mathcal{U}_{\lambda}^{0}(<1)$. Hence the inverse rescaling is directed by the gradient of $\tau$ and has universal rescaling functions.

(3) Let $\Sigma^{0}$ be the initial singularity of $\mathcal{U}_{\lambda}^{0}$. Then the map $d_{1}^{\lambda}$ extends to a continuous map

$$
\mathcal{U}_{\lambda}^{0}(\leq 1) \cup \Sigma^{0} \rightarrow \mathbb{X}_{1} \cup S_{\infty}^{2} .
$$

Moreover, its restriction to $\mathcal{U}_{\lambda}^{0}(1)$ coincides with $d_{\mathbb{H}}$; the restriction to $\Sigma^{0}$ is an (equivariant) isometry onto the initial singularity $\Sigma^{1}$ of $\mathcal{U}_{\lambda}^{1}$.

Note that, in contrast with the flat Lorentzian case, these de Sitter developing maps, as well as the dual hyperbolic ones are in general not injective.

4.7. Flat vs Anti de Sitter Lorentzian geometry. We are going to outline first a few features of the spacetimes in $\mathcal{M G H}_{-1}(S)$. Recall the content of Section 2.2, in 
particular the duality between points $x$ of $\mathbb{X}_{-1}$ and spacelike planes $P(x)$, or between spacelike lines, $l \rightarrow l^{*}$. Recall also that the boundary $\partial \mathbb{X}_{-1}$ has a natural causal structure, so that the notion of a nowhere timelike simple closed curve embedded in $\partial \mathbb{X}_{-1}$ makes sense.

Standard AdS spacetimes. Given such a curve $C \subset \mathbb{X}_{-1}$, assume furthermore that $C$ is different from a (left or right) leaf of the natural double foliation of $\partial \mathbb{X}_{-1}$ (that it is a so called admissible achronal curve). Then its Cauchy development is defined as

$$
\mathcal{Y}(C)=\left\{p \in \mathbb{X}_{-1} \mid \partial P(p) \cap C=\varnothing\right\}
$$

and the so obtained spacetime is called a (simply connected) standard AdS spacetime. $C$ is said its curve at infinity. $C$ is homotopic to the meridian of $\partial \mathbb{X}_{-1}$ with respect to $\mathbb{X}_{-1}$. In general and AdS spacetime is said standard if its universal covering is standard.

The convex core. There exists a spacelike plane $P$ not intersecting $\mathcal{Y}(C)$ (see [41]). In the Klein model we can cut $\mathbb{P}^{3}$ along the projective plane $\hat{P}$ containing $P$ and we have that $\mathcal{Y}(C)$ is contained in $\mathbb{R}^{3}=\mathbb{P}^{3} \backslash \hat{P}$. Since $C$ is nowhere timelike, then for every point $p \in C$ the plane $P(p)$ tangent to $\partial \mathbb{X}_{-1}$ at $p$ (that cuts $\mathbb{X}_{-1}$ at a null totally geodesic plane) does not separate $C$. It follows that the convex hull $\mathcal{K}(C)$ of $C$ in $\mathbb{R}^{3}$ is actually contained in $\mathbb{X}_{-1}$. We realize that $\mathcal{K}(C)$ does not depend on the choice of $\hat{P}$, and is called the convex core of $\mathcal{Y}(C)$.

Support planes of $\mathcal{K}(C)$ are non-timelike and the closure $\overline{\mathcal{Y}(C)}$ of $\mathcal{Y}(C)$ in $\mathbb{X}_{-1}$ coincides with the set of dual points of spacelike support planes of $\mathcal{K}(C)$ whereas the set of points dual to null support planes of $\mathcal{K}(C)$ coincides with $C . \overline{\mathcal{Y}(C)}$ is convex and the closure of $\mathcal{Y}(C)$ in $\overline{\mathbb{X}}_{-1}$ is $\overline{\mathcal{Y}(C)} \cup C$. It follows that $\mathcal{K}(C) \subset \overline{\mathcal{Y}(C)}$. A point $p \in \partial \mathcal{K}(C)$ lies in $\mathcal{Y}(C)$ if and only if it is touched only by spacelike support planes.

Being the boundary of a convex set in $\mathbb{R}^{3}, \partial \mathcal{K}(C) \cup C$ is homeomorphic to a sphere. In particular $\partial \mathcal{K}(C)$ (that is the boundary of $\mathcal{K}(C)$ in $\mathbb{X}_{-1}$ ) is obtained by removing a circle from a sphere, so it is the union of two disks. These components will be called the past and the future boundary of $\mathcal{K}(C)$ (with respect to the time orientation), and denoted $\partial_{-} \mathcal{K}(C)$ and $\partial_{+} \mathcal{K}(C)$ respectively. Given any inextendible timelike ray contained in $\mathcal{K}(C)$, its future end-point lies on the future boundary, and the past end-point lies on the past boundary.

$\partial_{+} \mathcal{K}(C) \cap \mathcal{Y}(C)$ is obtained by removing from $\partial_{+} \mathcal{K}(C)$ the set of points that admits a null support plane. Now suppose that a null support plane $P$ passes through $x \in$ $\partial_{+} \mathcal{K}(C)$. Then $P \cap \mathcal{K}(C)$ is a triangle with a vertex at $x(P)$, two ideal edges (that are segments on the leaves of the double foliation of $\left.\partial \mathbb{X}_{-1}\right)$ and a complete geodesic of $\mathcal{K}(C)$. It follows that the set $\partial_{+} \mathcal{K}(C) \cap \mathcal{Y}(C)$ is obtained by removing from $\partial_{+} \mathcal{K}(C)$ (at most) numerable many ideal triangles, so it is homeomorphic to a disk. The only case for $\partial_{+} \mathcal{K}(C) \cap \mathcal{Y}(C)$ to be empty is that the curve $C$ is obtained by joining the end-points of a spacelike geodesic $l$ with the end-points of its dual geodesic $l^{*}$; in that case $\mathcal{Y}(C)=\mathcal{K}(C)$, and we call it the degenerate standard spacetime. So, from now on, we incorporate in the definition of standard AdS spacetime that it is not degenerate. Moreover, since we will be mainly interested in $\partial_{+} \mathcal{K}(C) \cap \mathcal{Y}(C)$, from now on we will use $\partial_{+} \mathcal{K}(C)$ just to denote that set.

Proposition 4.13. $\partial_{+} \mathcal{K}(C)$ is locally $\mathrm{C}^{0}$-isometric to $\mathbb{H}^{2}$. 
Remark 4.14. If $\partial_{+} \mathcal{K}$ is complete then it is isometric to $\mathbb{H}^{2}$. In general $\partial_{+} \mathcal{K}$ is not complete, not even in the special case when $C$ is the graph of a homeomorphism of $S^{1}$ onto itself. Moreover, it can be not complete even when there are no null triangles on the boundary.

The past part of a standard spacetime. The past part $\mathcal{P}=\mathcal{P}(C)$ of a standard AdS spacetime $\mathcal{Y}(C)$ is the past in $\mathcal{Y}(C)$ of the future boundary $\partial_{+} \mathcal{K}$ of its convex core. The complement of $\partial_{+} \mathcal{K}$ in the frontier of $\mathcal{P}(C)$ in $\mathbb{X}_{-1}$ is called the past boundary of $\mathcal{Y}(C)$, denoted by $\partial_{-} \mathcal{P}$.

Proposition 4.15. Let $\mathcal{P}$ be the past part of some $\mathcal{Y}(C)$. Then $\mathcal{P}$ has cosmological time $\tau$ and this takes values on $(0, \pi / 2)$. For every point $p \in \mathcal{P}$ there exist only one point $\rho_{-}(p) \in \partial_{-} \mathcal{P}$, and only one point $\rho_{+}(p) \in \partial_{+} \mathcal{K}$ such that

1. $p$ is on the timelike segment joining $\rho_{-}(p)$ to $\rho_{+}(p)$.

2. $\tau(p)$ is equal to the length of the segment $\left[\rho_{-}(p), p\right]$.

3. the length of $\left[\rho_{-}(p), \rho_{+}(p)\right]$ is $\pi / 2$.

4. $P\left(\rho_{-}(p)\right)$ is a support plane for $\mathcal{P}$ passing through $\rho_{+}(p)$ and $P\left(\rho_{+}(p)\right)$ is a support plane for $\mathcal{P}$ passing through $\rho_{-}(p)$.

5. The map $p \mapsto \rho_{-}(p)$ is continuous. The function $\tau$ is $\mathrm{C}^{1}$ and its gradient at $p$ is the unit timelike tangent vector $\operatorname{grad} \tau(p)$ such that

$$
\exp _{p}(\tau(p) \operatorname{grad} \tau(p))=\rho_{-}(p) .
$$

Summing up, given the past part $\mathcal{P}$ of a standard AdS spacetime $\mathcal{Y}(C)$, we can construct:

the cosmological time $\tau: \mathcal{P} \rightarrow(0, \pi / 2)$;

the future retraction $\rho_{+}: \mathcal{P} \rightarrow \partial_{+} \mathcal{K}$;

the past retraction $\rho_{-}: \mathcal{P} \rightarrow \partial_{-} \mathcal{P}$.

Corollary 4.16. 1. Given $r$ in the past boundary of $\mathcal{Y}, \rho_{-}^{-1}(r)$ is the set of points $p$ such that the ray starting from $r$ towards $p$ meets at time $\pi / 2$ the future boundary of $\mathcal{K}$.

2. The image of $\rho_{-}$is the set of points of $\partial_{-} \mathcal{P}$ whose dual plane meets $C$ at least in two points.

3. The image of $\rho_{+}$is the whole $\partial_{+} \mathcal{K}$.

The image of the past retraction is called the initial singularity of $\mathcal{Y}(C)$.

For every surface of finite type $S$, $\operatorname{Stand}_{-1}(S)$ denotes the Teichmüller-like space of standard AdS spacetimes admitting a Cauchy surface homeomorphic to $S$. The following is a fundamental step towards the classification.

Theorem 4.17. $\operatorname{Stand}_{-1}(S)=\mathcal{M G H}_{-1}(S)$.

Note that a consequence of this theorem is that, similarly to the flat case, the developing maps of finite type $M G H$ AdS spacetimes are embedding onto convex domains. The fact that every spacetime in $\mathcal{M G H}_{-1}(S)$ is standard follows from the following more general result (Section 7 of [41]). 
Proposition 4.18. Let $Y$ be an Anti de Sitter simply connected spacetime, and $F \subset Y$ be a complete Cauchy surface. Then the developing map $Y \rightarrow \mathbb{X}_{-1}$ is an embedding onto a convex subset of $\mathbb{X}_{-1}$.

The closure of $F$ in $\overline{\mathbb{X}}_{0}$ is a closed disk and its boundary $\partial F$ is a nowhere timelike curve of $\partial \mathbb{X}_{-1}$.

If $Y$ is the maximal globally hyperbolic Anti de Sitter spacetime containing $F$ then $Y=\mathcal{Y}(C)$. The curve $\partial F$ determines $Y$, namely $p \in Y$ iff the dual plane $P(p)$ does not meet $\partial F$.

Conversely $\partial F$ is determined by $Y$, in fact $\partial F$ is the set of accumulation points of $Y$ on $\partial \mathbb{X}_{-1}$. If $F^{\prime}$ is another complete spacelike Cauchy surface of $Y$ then $\partial F^{\prime}=\partial F$.

The main step in order to prove the opposite inclusion is the following proposition (recently achieved also by Barbot [6](2) with a different approach with respect to [10]), that also holds for arbitrary standard spacetimes.

Proposition 4.19. If $\mathcal{P}$ is the past part of $\mathcal{Y}(C)$ then every level surface $\mathcal{P}(a)$ of the cosmological time is complete.

Corollary 4.20. Every level surface $\mathcal{P}(a)$ of the past part $\mathcal{P}$ of a standard AdS spacetime $\mathcal{Y}(C)$ is a complete Cauchy surface of $\mathcal{Y}(C)$ and this last is the maximal globally hyperbolic AdS spacetime that extends $\mathcal{P}$.

Remark 4.21. $\tau$ extends to the cosmological time of $\mathcal{Y}(C)$, that takes values on some interval $\left(0, a_{0}(C)\right)$, for some well defined $\pi / 2<a_{0}(C)<\pi$. Notice however that $\tau$ is $\mathrm{C}^{1}$ only on the past part.

The map $\mathfrak{m}_{-1}$. Let $(F, \lambda) \in \mathcal{M L}(S), F \subset F^{\mathcal{C}} \subset \hat{F}=\mathbb{H}^{2} / \Gamma\left(F^{\mathcal{C}}, \lambda\right)$, with universal coverings $\mathbb{H}^{2} \rightarrow \hat{F}, H \rightarrow F^{\mathcal{C}}$, and $\stackrel{\circ}{H} \rightarrow F$ respectively, as usual. Fix an embedding of $\mathbb{H}^{2}$ in $\mathbb{X}_{-1}$ as a spacelike plane (for instance as $P(I d)$ ). The key ingredient to construct $\mathfrak{m}_{-1}$ is the AdS version of the bending of $H \subset \mathbb{H}^{2}$ along the lamination $\lambda$ (see

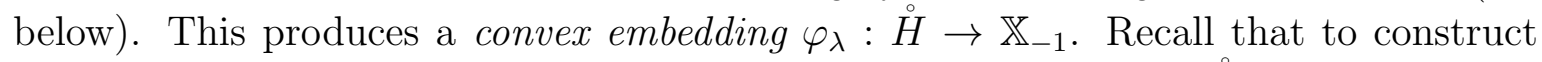
the $H$-hull (via the Wick rotation) we used the bending map $f_{\lambda}: \stackrel{\circ}{H} \rightarrow \mathbb{H}^{3}$, that is a local convex embedding, and then we followed the geodesic rays normal to $f_{\lambda}(\stackrel{\circ}{H})$, in the non-convex side bounded by $f_{\lambda}(\stackrel{\circ}{H})$. Eventually the developing map $d_{\mathbb{H}}$ has been obtained by requiring that the integral lines of the cosmological times would be sent to the integral lines of the normal flow. Also in the present situation we construct a $\mathrm{C}^{1}$ developing map

$$
d_{-1}^{\lambda}: \mathcal{U}_{0}^{\lambda} \rightarrow \mathbb{X}_{-1}
$$

by requiring that the integral lines of the cosmological time of $\mathcal{U}_{\lambda}^{0}$ are sent to the integral line of the normal flow. An important difference, with respect to the hyperbolic case, is that the normal flow is followed now in the convex side bounded by $\varphi_{\lambda}(\stackrel{\leftrightarrow}{H})$ (otherwise singularities would be reached). It turns that the image of $d_{-1}^{\lambda}$ is the past part of a standard AdS spacetime, that plays here the role of a sort of AdS-hull. More precisely we have:

Theorem 4.22. (1) $d_{-1}^{\lambda}$ is an embedding onto the past part $\mathcal{P}^{\lambda}$ of a determined $\mathcal{U}_{-1}^{\lambda}=$ $\mathcal{Y}\left(C^{\lambda}\right)$, which is the universal covering of $Y_{-1}^{\lambda} \in \mathcal{M G H}_{-1}(S)$. The image of the AdS 
bending map $\varphi_{\lambda}$ coincides with $\partial_{+} \mathcal{K}\left(C^{\lambda}\right)$. The map $d_{-1}^{\lambda}$ continuously extends to an isometry between the respective initial singularity.

(2) $\mathcal{U}_{-1}^{\lambda}$ is produced by the rescaling of $\mathcal{U}_{0}^{\lambda}$, directed by the gradient of the cosmological time $T$, with universal rescaling functions

$$
\alpha=\frac{1}{1+T^{2}}, \quad \beta=\frac{1}{\left(1+T^{2}\right)^{2}} .
$$

(3) The cosmological time $\tau$ on $\mathcal{P}^{\lambda}$ is given by

$$
T(p)=\tan \tau(p) .
$$

In such a way we construct an injective map

$$
\mathfrak{m}_{-1}: \mathcal{M L}(S) \rightarrow \mathcal{M G H}_{-1}(S)
$$

The following general proposition (specialized to $\left.\operatorname{Stand}_{-1}(S)\right)$ implies that $\mathfrak{m}_{-1}$ is in fact a bijection.

Proposition 4.23. For every standard $A d S$ domain $\mathcal{Y}=\mathcal{Y}(C)$, the rescaling of its past part $\mathcal{P}$, directed by the gradient of the cosmological time $\tau$, with universal rescaling functions

$$
\alpha=\frac{1}{\cos ^{2} \tau} \quad \beta=\frac{1}{\cos ^{4} \tau}
$$

produces a regular domain, whose cosmological time is given by the formula

$$
T=\tan \tau .
$$

It is not too hard to see, by means of local considerations, that such a rescaling produces a flat spacetime. Showing that it is regular domain is actually more demanding. This is equivalent to show that the future boundary of the convex core is isometric to a straight convex set pleated at a measured lamination. The key point is the fact that level surfaces of $\tau$ are complete (Proposition 4.20).

On AdS bending. We are going to outline more precisely the construction of $d_{-1}^{\lambda}$. The AdS bending runs similarly to the hyperbolic one, but having some remarkable differences (that are eventually responsible, for example, that the AdS developing maps are embeddings, in contrast with the hyperbolic ones). We also stress that orientations play a subtle role in the AdS bending procedure. The basic diffence arises from the different behaviour of the "angles" between hyperbolic planes (that is spacelike planes) and of "rotations" around spacelike geodesics in $\mathbb{X}_{-1}$, with respect to $\mathbb{H}^{3}$. In fact, given two spacelike planes $P_{1}, P_{2}$ meeting each other along a geodesic $l$, the dual points $x_{i}=x\left(P_{i}\right)$ lie on the geodesic $l^{*}$ dual to $l$. Then we define the angle between $P_{1}$ and $P_{2}$ as the distance between $x_{1}$ and $x_{2}$ along $l^{*}$. Notice that:

Fix $P_{1}$, the by varying $P_{2}$, the angles between them are well defined numbers that span the whole of the interval $(0,+\infty)$.

Define a rotation around a spacelike geodesic $l$ simply to be an isometry of $\mathbb{X}_{-1}$ which point-wise fixes $l$. We have

Lemma 4.24. Rotations around a geodesic $l$ act freely and transitively on the dual geodesic $l^{*}$. Such action induces an isomorphism between the set of rotations around $l$ and the set of translations of $l^{*}$. 


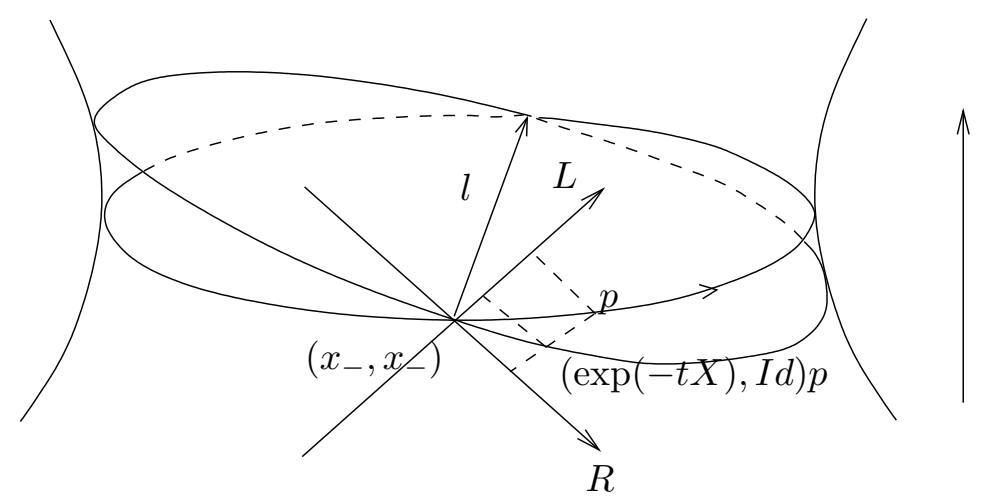

Figure 5. $(\exp (-t X), I d)$ rotates planes around $l$ in the positive sense.

By duality, rotations around $l$ act freely and transitively on the set of spacelike planes containing $l$. Given two spacelike planes $P_{1}, P_{2}$ such that $l \subset P_{i}$, then there exists a unique rotation $T_{1,2}$ around $l$ such that $T_{1,2}\left(P_{1}\right)=P_{2}$.

Lemma 4.25. An isometry of $\mathbb{X}_{-1}$ is a rotation around a geodesic if and only if it is represented by a pair $(x, y)$ such that $x$ and $y$ are isometries of $\mathbb{H}^{2}$ of hyperbolic type with the same translation length.

Given two spacelike planes $P_{1}, P_{2}$ meeting along a geodesic $l$, let $(x, y)$ be the rotation taking $P_{1}$ to $P_{2}$. Then the translation length $\tau$ of $x$ coincides with the angle between $P_{1}$ and $P_{2}$.

There is a natural definition of positive rotation around an oriented spacelike geodesic $l$ (depending only on the orientations of $l$ and $\mathbb{X}_{-1}$ ). Thus, an orientation on the dual line $l^{*}$ is induced by requiring that positive rotations act by positive translations on $l^{*}$. In particular, if we take an oriented geodesic $l$ in $P(I d)$, and denote by $X$ the infinitesimal generator of positive translations along $l$ then it is not difficult to show that the positive rotations around $l$ are of the form $(\exp (-t X), \exp (t X))$ for $t>0$. Actually, by looking at the action on the boundary we deduce that both the maps $(\exp (-t X), I d)$ and $(I d, \exp (t X))$ rotate planes through $l$ in the positive direction (see Fig. 51).

Given $\lambda$ on $H$ as usual, we construct now an AdS bending cocycle

$$
B^{\lambda}=\left(B_{-}^{\lambda}, B_{+}^{\lambda}\right): \stackrel{\circ}{H} \times \stackrel{\circ}{H} \rightarrow P S L(2, \mathbb{R}) \times P S L(2, \mathbb{R})
$$

which formally satisfies the similar properties like the quake cocycles of Section 3.6, or the above hyperbolic bending cocycle. In fact $B_{-}^{\lambda}$ and $B_{+}^{\lambda}$ are exactly the EpsteinMarden cocycles (like the quake cocycles), corresponding to the real-valued measured laminations $-\lambda$ and $\lambda$. Here $-\lambda=(L,-\mu)$, that is we take the negative-valued measure $-\mu$. Although this is no longer a measured lamination in the ordinary sense, the construction of [24] does apply. Besides the usual cocycle properties, $B^{\lambda}$ also verifies that if $x, y$ lie in different strata then $B_{+}^{\lambda}(x, y)$ (resp. $\left.B_{-}^{\lambda}(x, y)\right)$ is a non-trivial hyperbolic transformation whose axis separates the stratum through $x$ and the stratum through $y$. Moreover the translation length is bigger than the total mass of $[x, y]$. 
All this is very simple on the usual local model for finite laminations. In fact, take take a finite measured geodesic lamination $\lambda$ of $\mathbb{H}^{2}$. Take a pair of points $x, y \in \mathbb{H}^{2}$ and enumerate the geodesics in $\lambda$ that cut the segment $[x, y]$ in the natural way $l_{1}, \ldots, l_{n}$. Moreover, we can orient $l_{i}$ as the boundary of the half-plane containing $x$. With a little abuse, denote by $l_{i}$ also the geodesic in $P(I d)$ corresponding to $l_{i}$, then let $B^{\lambda}(x, y)$ be the isometry of $\mathbb{X}_{-1}$ obtained by composition of positive rotations around $l_{i}$ of angle $a_{i}$ equal to the weight of $l_{i}$. In particular, if $X_{i}$ denotes the unit positive generator of the hyperbolic transformations with axis equal to $l_{i}$, then we have

$$
\begin{aligned}
& B_{\lambda}(x, y)=\left(B_{-}^{\lambda}(x, y), B_{+}^{\lambda}(x, y)\right) \in P S L(2, \mathbb{R}) \times P S L(2, \mathbb{R}) \quad \text { where } \\
& B_{-}^{\lambda}(x, y)=\exp \left(-a_{1} X_{1} / 2\right) \circ \exp \left(-a_{2} X_{2} / 2\right) \circ \ldots \circ \exp \left(-a_{n} X_{n} / 2\right) \\
& B_{+}^{\lambda}(x, y)=\exp \left(a_{1} X_{1} / 2\right) \circ \exp \left(a_{2} X_{2} / 2\right) \circ \ldots \circ \exp \left(a_{n} X_{n} / 2\right)
\end{aligned}
$$

with the following possible modifications: $a_{1}$ is replaced by $a_{1} / 2$ when $x$ lies on $l_{1}$ and $a_{n}$ is replaced by $a_{n} / 2$ when $y$ lies on $l_{n}$ The factor $1 / 2$ in the definition of $\beta_{ \pm}$arises because the translation length of $\exp t X$ is $2 t$.

By means of the bending cocycle we construct a $A d S$ bending map: take a base point $x_{0}$ in $\stackrel{\circ}{H}$ and set

$$
\varphi_{\lambda}: \stackrel{\circ}{H} \ni x \mapsto B^{\lambda}\left(x_{0}, x\right) x
$$

Proposition 4.26. The bending map $\varphi_{\lambda}$ is an isometric $\mathrm{C}^{0}$ embedding of $\stackrel{\circ}{H}$ onto an achronal set $\mathbb{X}_{-1}$.

Let $\mathcal{U}=\mathcal{U}_{\lambda}^{0}$ be the flat spacetime encoded by $\lambda$. Just as in the hyperbolic case we want to "pull-back" the bending cocycle $B^{\lambda}$ to a continuous bending cocycle

$$
\hat{B}^{\lambda}: \mathcal{U} \times \mathcal{U} \rightarrow P S L(2, \mathbb{R}) \times P S L(2, \mathbb{R}) .
$$

In fact we get a natural extension such that:

(1) For every $p, q \in \mathcal{U}$ such that $N(p)$ and $N(q)$ do not lie on weighted part of the lamination, then

$$
\hat{B}^{\lambda}(p, q)=B^{\lambda}(N(p), N(q)) .
$$

(2) $\hat{B}^{\lambda}$ on the whole of $\mathcal{U}$ is constant along the integral geodesics of the gradient of the cosmological time $T$.

(3) It is locally Lipschitz (with respect to the Euclidean distance on $\mathcal{U}$ ), and the the Lipschitz constant on $K \times K(K$ being any compact set in $\mathcal{U})$ depends only on the image of the Gauss map $N(K)$, the maximum of the total masses of geodesic paths of $H$ joining points in $N(K)$, and the maximum and the minimum of the cosmological time $T$ on $K$.

Finally we can define our developing map $d_{-1}^{\lambda}: \mathcal{U}_{0}^{\lambda} \rightarrow \mathbb{X}_{-1}$. For every $p \in \mathcal{U}_{\lambda}^{0}$, we define $x_{-}(p)$ as the dual point of the plane $\hat{B}^{\lambda}\left(p_{0}, p\right)(P(I d))$ (that is $\hat{B}^{\lambda}\left(p_{0}, p\right)(I d)$, and $x_{+}(p)=\hat{B}^{\lambda}\left(p_{0}, p\right)(N(p))$. Take representatives $\hat{x}_{-}(p)$ and $\hat{x}_{+}(p)$ in $\operatorname{SL}(2, \mathbb{R})$ such that the geodesic segment between $\hat{x}_{-}(p)$ and $\hat{x}_{+}(p)$, is future directed. Finally set

$$
d_{-1}^{\lambda}(p)=\left[\cos \tau(p) \hat{x}_{-}(p)+\sin \tau(p) \hat{x}_{+}(p)\right]
$$

where $\tau(p)=\arctan T(p)$.

As usual, we end with a few explicit computations for our favourite local model, that is when when $\lambda$ is a single weighted geodesic. 


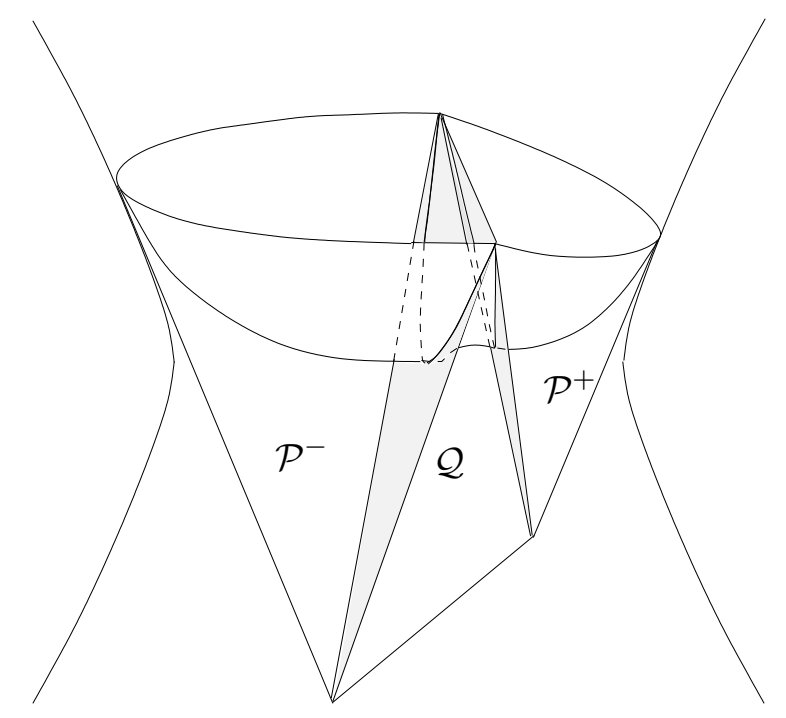

Figure 6. The domain $\mathcal{P}$ with its decomposition. Also the surface $\mathcal{P}(a)$ is shown.

Let us set $\lambda_{0}=\left(l_{0}, a_{0}\right)$ and choose a base point $p_{0} \in \mathbb{H}^{2}-l_{0}$. The surface $P=\varphi_{\lambda}\left(\mathbb{H}^{2}\right)$ is simply the union of two half-planes $P_{-}$and $P_{+}$meeting each other along a geodesic (that, with a little abuse of notation, is denoted by $l_{0}$ ). We can suppose that $p_{0}$ is in $P_{-}$, and $l_{0}$ is oriented as the boundary of $P_{-}$. If $v_{ \pm}$denote the dual points of the planes containing $P_{ \pm}$we have $v_{-}=I d$ and $v_{+}=\exp -a_{0} X_{0}, X_{0}$ being the standard generator of translations along $l_{0}$. The vector $X_{0}$ is tangent to $P(i d)$ along $l_{0}$, orthogonal to it, and points towards $p_{0}$.

By definition, the image, say $\mathcal{P}$, of $\Delta_{0}=d_{-1}^{\lambda_{0}}$ is the union of three pieces: the cone with vertex at $v_{-}$and basis $P_{-}$, say $\mathcal{P}_{-}$, the cone with vertex at $v_{+}$and basis $\mathcal{P}_{+}$, and the join of the geodesic $l_{0}$ and the segment $\left[v_{-}, v_{+}\right]$, say $\mathcal{Q}$.

Fix a point in $l_{0}$, say $p_{0}$, and denote by $v_{0}$ the unit tangent vector of $l_{0}$ at $p_{0}$ (that we will identify with a matrix in $M(2, \mathbb{R}))$. Consider the coordinates on $\mathcal{U}_{0}$, say $(T, u, \zeta)$ introduced in Section 4.5. With respect to these coordinates we have $\Delta_{0}(T, u, \zeta)= \begin{cases}\sin \tau\left(\operatorname{ch} \zeta\left(\operatorname{ch} u \hat{p}_{0}+\operatorname{sh} u v_{0}\right)-\operatorname{sh} \zeta X_{0}\right)+\cos \tau \hat{v}_{-} & \text {if } \zeta<0 \\ \sin \tau\left(\operatorname{ch} u \hat{p}_{0}+\operatorname{sh} u v_{0}\right)+\cos \tau \exp \left(-\zeta \tan \tau X_{0}\right) & \text { if } \zeta \in\left[0, a_{0} / T\right] \\ \sin \tau\left(\operatorname{ch} \zeta^{\prime}\left(\operatorname{ch} u \hat{p}_{0}+\operatorname{sh} u v_{0}\right)-\operatorname{sh} \zeta^{\prime} X_{0}\right)+\cos \tau \hat{v}_{+} & \text {otherwise }\end{cases}$ where $\zeta^{\prime}=\zeta-a_{0} / T, \tau=\arctan T$ and $\hat{p}_{0}, \hat{v}_{+}, \hat{v}_{-} \in S L(2, \mathbb{R})$ are chosen as above. Clearly $\Delta_{0}$ is $\mathrm{C}^{\infty}$ for $\zeta \neq 0, a_{0} / T$. A direct computation shows that the derivatives along the coordinate fields glue on $\zeta=0$ and $\zeta=a_{0} / T$ and this proves that $\Delta_{0}$ is $\mathrm{C}^{1}$. By a direct computation we have

$$
\Delta_{0}^{*}(\eta)= \begin{cases}-\mathrm{d} \tau^{2}+\sin ^{2} \tau\left(\mathrm{d} \zeta^{2}+\operatorname{ch}^{2} \zeta \mathrm{d} u^{2}\right) & \text { if } \zeta<0 \\ -\mathrm{d} \tau^{2}+\sin ^{2} \tau\left(\mathrm{d} \zeta^{2}+\mathrm{d} u^{2}\right) & \text { if } \zeta \in\left[0, a_{0} / T\right] \\ -\mathrm{d} \tau^{2}+\sin ^{2} \tau\left(\mathrm{d} \zeta^{2}+\operatorname{ch}^{2} \zeta^{\prime} \mathrm{d} u^{2}\right) & \text { otherwise }\end{cases}
$$

Since $\mathrm{d} \tau^{2}=\frac{1}{\left(1+T^{2}\right)^{2}}$ and $\sin ^{2} \tau=\frac{T^{2}}{1+T^{2}}$, we finally see that $\Delta_{0}$ is obtained by a rescaling directed by the gradient of $T$ with the right rescaling functions. 
Compatible holonomy. The holonomy representation of $Y_{-1}^{\lambda} \in \mathcal{M G} \mathcal{L}(S), h_{-1}^{\lambda}$ : $\pi_{1}(S) \rightarrow P S L(2, \mathbb{R}) \times P S L(2, \mathbb{R})$, compatible with $d_{-1}^{\lambda}$ is obtained as follows. If $x_{0} \in \stackrel{\circ}{H}$ is the usual fixed base point of the construction, then for every $\gamma \in \pi_{1}(S)=\Gamma$

$$
h_{-1}^{\lambda}(\gamma)=B^{\lambda}\left(x_{0}, \gamma x_{0}\right) \circ(\gamma, \gamma) .
$$

Remark 4.27. It follows from the previous discussion, that the spacetimes in $\mathcal{M G H}_{-1}(S)$ have a few analogies with the hyperbolic 3-manifolds arising as $H$-hulls of quasiFuchsian projective surfaces belonging to $\mathcal{P}(S)$. For instance the curves at infinity $C \subset \partial \mathbb{X}_{-1}$ of $\mathcal{Y}(C)$ play a similar rôle of the Jordan curves that bound the universal coverings embedded in $\partial \mathbb{H}^{3} S_{\infty}^{2}$ of quasi-Fuchsian surfaces. However, there are important difference that make the AdS behaviour much more "tame". For example such Jordan curves are in general rather wild, while the curves $C$ are Lipschitz. Moreover, taking for example $S$ compact, for every $(F, \lambda) \in \mathcal{M L}(S)$, along the ray $(F, t \lambda)$ there is a critical value $t_{0}>0$ such that $S^{t \lambda} \in \mathcal{P}(S)$ is quasi-Fuchsian only for $t<t_{0}$. On the other hand, the description of $Y^{t \lambda}$ is qualitatively the same for every $t>0$; in particular all AdS developing maps are embeddings.

\section{Causal AdS spacetimes, earthquakes and Black holes}

Beyond the classification achieved in the previous Section, the AdS case displays a rich phenomenology that we are going to point out.

5.1. On holonomy pregnancy. Let us recall first the following results of [41], in the case of compact $S$.

Theorem 5.1. If $S$ is compact, and $Y \in \mathcal{M G H}_{-1}(S)$, then:

(a) The holonomy $h=\left(h_{L}, h_{R}\right)$ of $Y$ is made by a couple of Fuchsian representations of $\pi_{1}(S)$, and every such a couple arises in this way (by varying $Y$ ).

(b) $Y$ is completely determined by its holonomy $h=\left(h_{L}, h_{R}\right)$. In fact $Y=\mathcal{Y}(C)$, where $C$ is the graph in $S_{\infty}^{1} \times S_{\infty}^{1}=\partial \mathbb{X}_{-1}$ of the unique orientation preserving homeomorphism that conjugates the action of $h_{L}$ on $S_{\infty}^{1}=\partial \mathbb{H}^{2}$ with the one of $h_{R}$. This curve $C$ is the unique $h$-invariant curve on $\partial \mathbb{X}_{-1}$.

In $[6](2,3)$ we can find the following generalization of point (a). Here we use the notations of Section 3 .

Proposition 5.2. Let $Y \in \mathcal{M G H}_{-1}(S)$ be of finite type, with holonomy representation

$$
h=\left(h_{L}, h_{R}\right): \pi_{1}(Y) \rightarrow \operatorname{PSL}(2, \mathbb{R}) \times \operatorname{PSL}(2, \mathbb{R}) .
$$

Then both $h_{L}$ and $h_{R}$ are holonomy representations of hyperbolic structures belonging to $\mathcal{T}(S)$. Conversely given a pair of representations $h=\left(h_{L}, h_{R}\right)$ corresponding to elements of $\mathcal{T}(S)$, then there exists a spacetime $Y \in \mathcal{M G H}_{-1}(S)$ whose holonomy is $h$.

Concerning point (b), the following partial generalization holds. Here we adopt the notations of Corollary 3.31 .

Proposition 5.3. $Y \in \mathcal{M G H}_{-1}(S)$ is completely determined by its holonomy providing that $Y=Y_{-1}^{\lambda}$, for some $(F, \lambda) \in \mathcal{V}_{\mathfrak{c}}(F) \cap \mathcal{M L}_{\mathfrak{c}}(F)^{0}$. 
This is essentially a consequence of the proof of the Earthquake Theorem considered below. On the other hand, non-equivalent spacetimes in $\mathcal{M G H}_{-1}(S)$ can actually share the same holonomy. This is the theme of the following construction.

5.2. Canonical causal AdS spacetimes with prescribed holonomy. We mostly refer to [6] $(2,3)$. Let us fix a representation $h=\left(h_{L}, h_{R}\right)$ of $\pi_{1}\left(S, p_{0}\right)$ as in Theorem 5.2. We stress that the representation is fixed, not only its conjugation class; for this reason we have also fixed a base point $p_{0} \in S$. We consider the domain

\section{$\tilde{\Omega}(h)$}

of points $x \in \mathbb{X}_{-1}$ such that, for every $\gamma \in \pi_{1}\left(S, p_{0}\right), x$ and $h(\gamma)(x)$ are not causally related. We have:

Proposition 5.4. $\tilde{\Omega}(h)$ is simply connected and h-invariant; the action of $\pi_{1}\left(S, p_{0}\right)$ on it is free and properly discontinuous. The quotient, say $\Omega(h)$, is a causal AdS spacetime homeomorphic to $S \times \mathbb{R}$.

Let us consider now

$$
\mathcal{M G} \mathcal{L}(h)
$$

the set of all AdS $M G H$ spacetimes $Y$ homeomorphic to $S \times \mathbb{R}$, determined by a compatible couple of developing map and holonomy representation $\left(d_{Y}, h_{Y}\right)$ such that $h_{Y}=h$. Equivalently, we are considering spacetimes homeomorphic to $S \times \mathbb{R}$ of the form $Y=\mathcal{Y}(C) / h$ such that the nowhere time-like curve at infinity $C$ is $h$-invariant. Note again that each such a maximal globally hyperbolic spacetime is fixed and not considered up to Teichmüller-like equivalence. However, it is not hard to see that:

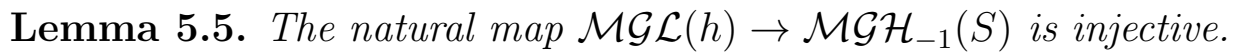

Remark 5.6. If $h^{\prime}=g h g^{-1}$ is conjugate to $h$, then $\tilde{\Omega}\left(h^{\prime}\right)=g \tilde{\Omega}(h)$, as well $\mathcal{M G} \mathcal{L}\left(h^{\prime}\right)=$ $g \mathcal{M G L}(h)$, so that they have the same image in $\mathcal{M G H}_{-1}(S)$. By fixing a representative $h$ in any conjugation class, we get in this way a partition of $\mathcal{M G H}_{-1}(S)$.

Since every $Y \in \mathcal{M G} \mathcal{L}(h)$ is causal, we have

Lemma 5.7. There is a natural h-invariant embedding of $\mathcal{Y}(C)$ in $\tilde{\Omega}(h)$, hence of $Y$ in $\Omega(h)$.

In fact we can prove

Proposition 5.8. $\Omega(h)$ is the union of the $Y \in \mathcal{M G} \mathcal{L}(h)$, as well as $\tilde{\Omega}(h)$ is the union of the h-invariant $\mathcal{Y}(C)$ 's.

See below for a description of the curves $C$ arising in this way.

$\partial_{\infty} \tilde{\Omega}(h)$ and the limit set. The inclusion of Lemma 5.7 is in general strict, in particular $\Omega(h)$ can be not globally hyperbolic. In a sense, it is just the maximal causal extension of every globally hyperbolic $Y \in \mathcal{M G} \mathcal{L}(h)$. The reason is that the adherence,

$$
\partial_{\infty} \tilde{\Omega}(h)
$$

of $\tilde{\Omega}(h)$ on the boundary of $\mathbb{X}_{-1}$ can have non-empty interior part. Such an adherence can be explicitly described by means of the holonomy of the peripheral loops. Let $\gamma \in \pi_{1}\left(S, p_{0}\right)$ freely homotopic to a loop surrounding a point in $V$. If $h_{L}(\gamma)$ (resp. 
$\left.h_{R}(\gamma)\right)$ is of hyperbolic type, we set $I_{L}(\gamma)$ (resp. $\left.I_{R}(\gamma)\right)$ to be the interval of $S^{1}$ whose end-points are the fixed points of $h_{L}(\gamma)$ (resp. $\left.h_{R}(\gamma)\right)$ and that does not meet the limit set of $h_{L}(\gamma)$ (resp. $h_{R}(\gamma)$ ). If $h_{L}(\gamma)$ is parabolic, then let $I_{L}(\gamma)$ be the fixed point of $h_{L}(\gamma)$. Similarly for $I_{R}(\gamma)$. Then the "rectangle" $R(\gamma)=I_{L}(\gamma) \times I_{R}(\gamma)$ is contained in the adherence of $\tilde{\Omega}(h)$, and in fact $\partial_{\infty} \tilde{\Omega}(h)$ is the closure $\bigcup_{\gamma} R(\gamma)$. The closure of the complement of the union of these rectangles $R(\gamma)$ in $\partial_{\infty} \tilde{\Omega}(h)$ can be regarded as a limit set

$$
\Lambda=\Lambda(h)
$$

in the sense that it is contained in the closure of the orbit of any point $x \in \overline{\mathbb{X}}_{-1}$. A rectangle is non-degenerate if both $h_{L}(\gamma), h_{R}(\gamma)$ are of hyperbolic type.

Lemma 5.9. If some rectangle $R(\gamma)$ is non-degenerate, then the interior of $\partial_{\infty} \tilde{\Omega}(h)$ is not empty. $\Omega(h)$ is not globally hyperbolic if and only if there is some non-degenerate rectangles .

For it is possible to find points $p, q$ close to $R(\gamma)$ such that $\mathrm{I}^{+}(p) \cap \mathrm{I}^{-}(q)$ is not precompact in $\mathbb{X}_{-1}$ and this contradicts the global hyperbolicity.

The asymptotic regions. Notice that a non degenerate rectangle $R(\gamma)$ has exactly two vertices that are the end-points of a spacelike geodesic $l_{\gamma}$ that is invariant for $\left(h_{L}(\gamma), h_{R}(\gamma)\right)$. The boundary lines of $R(\gamma)$ together with $l_{\gamma}$ span a surface $\tilde{H}(\gamma)$ embedded in $\tilde{\Omega}(h)$ made by two null triangles intersecting at $l_{\gamma}$. This surface divides $\tilde{\Omega}(h)$ in two components. The component whose adherence in $\partial_{\infty} \tilde{\Omega}(h)$ is $R(\gamma)$ is called an asymptotic region of $\tilde{\Omega}(h)$, denoted by $\tilde{A}(\gamma), \tilde{H}(\gamma)$ is its horizon. $(A(\gamma), H(\gamma))$ is invariant for $\left(h_{L}(\gamma), h_{R}(\gamma)\right)$ and the quotient embeds in $\Omega(h)$ giving us an asymptotic region $A(\gamma)$ with horizon $H(\gamma)$. This last is the union of two null annuli along a spacelike closed geodesic. The length of this spacelike geodesic is called the size of the horizon, whereas the momentum is the twist factor for the parallel transport along it. If $l_{L}, l_{R}$ are the translation lengths of $h_{L}(\gamma)$ and $h_{R}(\gamma)$, the size is simply

$$
s=\left(l_{L}+l_{R}\right) / 2
$$

whereas the momentum is

$$
m=\left(l_{L}-l_{R}\right) / 2 .
$$

$\Omega(h)$ has exactly $k$ asymptotic regions, where $k$ is the number of points $p \in V$ such that the surrounding circle is of hyperbolic type for both $h_{L}$ and $h_{R}$.

More about $\mathcal{Y}(C) \subset \tilde{\Omega}(h)$. Clearly the $h$-invariant curve at infinity $C$ is contained in $\partial_{\infty} \tilde{\Omega}(h)$. On the other hand, every nowhere time-like meridian of $\partial \mathbb{X}_{-1}$ contained in $\partial_{\infty} \tilde{\Omega}(h)$ is determined by drawing in each non-degenerate rectangle $R(\gamma)$ an arc $l_{\gamma}$ joining the vertices that are the end-points of the spacelike geodesic of the corresponding horizon. In the degenerate case the segment $l_{\gamma}$ coincide with $R(\gamma)$. The closure $C$ of the union of these $l_{\gamma}$ 's is a nowhere timelike meridian; moreover, if the segments are chosen in $h$-invariant way (that is $l_{\alpha \gamma \alpha^{-1}}=h(\alpha) l_{\gamma}$ ), then $C$ is is the curve at infinity of the universal covering of some $Y \subset \Omega(h)$. 
5.3. AdS bending and Earthquake Theorems. By extending the arguments given in [41] in the case of compact $S$, we have for a general $S$ of finite type:

Proposition 5.10. Let $Y \in \mathcal{M G H}_{-1}(S)$ be encoded by $(F, \lambda) \in \mathcal{M L}(S)$, and $h=$ $\left(h_{L}, h_{R}\right)$ be its holonomy. Then $h_{L}\left(h_{R}\right)$ is the holonomy of the surface $F_{L}=\beta_{\lambda}^{L}(F)$ $\left(F_{R}=\beta_{\lambda}^{R}(F)\right)$, that is the surface in $\tilde{\mathcal{T}}(S)$ obtained by the left (right) earthquake on $F$ along $\lambda$.

We stress that Proposition 5.10, together with Theorem 5.1, actually give an AdS proof of the Earthquake Theorem 3.33 when $S$ is compact. For, given $F^{0}, F^{1}$ two hyperbolic structures on a compact surface $S$, there exists a unique spacetime $Y_{-1}^{\lambda} \in \mathcal{M G \mathcal { H }} \mathcal{H}_{-1}(S)$ whose holonomy is $h=\left(h^{0}, h^{1}\right)$, where $h^{j}$ is the hyperbolic holonomy of $F^{j}$. Then the left earthquake along $2 \lambda$ transforms $F^{0}$ into $F^{1}$.

We consider the subset

$$
\mathcal{M G H}_{\mathfrak{c}}(h)
$$

of $\mathcal{M G \mathcal { H }}(h)$ consisting of the spacetimes $Y$ that satisfy the further condition of being encoded by couples $(F, \lambda) \in \mathcal{M} \mathcal{L}_{\mathfrak{c}}(S)$. In order to get such an AdS proof of the full Earthquake Theorem 3.33, we need to characterize the spacetimes $Y=\mathcal{Y}(C) / h \in$ $\mathcal{M G H}_{\mathfrak{c}}(h)$ in terms of the curve at infinity $C$. Consider again the general description of an $h$-invariant meridian $C$ given above. A case of particular interest is when the segments $l_{\gamma}$ are chosen on the boundary of $R(\gamma)$. Meridians $C$ obtained in this way are called extremal. Notice that for each asymptotic region there are only two ways to chose such an arc: an upper extremal arc and a lower extremal arc. Thus, there are exactly $2^{k} h$-invariant extremal arcs where $k$ is defined as above. This holds also when $k=0$; in such a case $\Omega(h)=\mathcal{Y}(C) / h$ is globally hyperbolic, and $C$ is its extremal meridian. Finally we the following nice geometric characterization (see [19]).

Proposition 5.11. $\mathcal{Y}(C)$ is the universal covering of some $Y \in \mathcal{M G H}_{\mathfrak{c}}(h)$ if and only if $C$ is an h-invariant extremal meridians.

Corollary 5.12. $\Omega(h)$ is globally hyperbolic if and only if it belongs to $\mathcal{M G H}_{\mathfrak{c}}(h)$ and is encoded by $(F, \lambda)$ such that $F \in \mathcal{T}_{g, r}$ and the lamination does not enter the cusps

We are ready to prove Theorem 3.33. Let $F^{0}, F^{1}$ be the the interior of the convex cores of $\mathbb{H}^{2} / h^{0}, \mathbb{H}^{2} / h^{1}$ respectively, that are both homeomorphic to $S$. Set $h=\left(h^{0}, h^{1}\right)$ and take $\Omega(h)$. Let us apply Proposition 5.10 to every $Y \in \mathcal{M G \mathcal { G }}(h)$, encoded by some $(F, \lambda) \in \mathcal{M L}_{\mathfrak{c}}(S)$. As the convex cores are uniquely determined by the holonomy, and $\mathcal{T}_{\mathfrak{c}}(S)$ is closed under earthquakes, it follows that $F^{0}=\beta_{\lambda}^{L}(F), F^{1}=\beta_{\lambda}^{R}(F)$, so that $F^{1}=\beta_{2 \lambda}^{L}\left(F^{0}\right)$. The determined lack of uniqueness in Theorem 3.33, the "enhanced" version 3.34, as well as Corollary 3.35 are now rather easy consequences of Proposition 5.11, Lemma 3.28, and the definition of the (enhanced) quake-flow.

5.4. Convex core of $\Omega(h)$ and black hole. We denote by

$$
\Omega_{\mathfrak{c}}(h) \subset \Omega(h)
$$

the union of the spacetimes belonging to $\mathcal{M G H}_{\mathfrak{c}}(h)$. We do similarly for $\tilde{\Omega}_{\mathfrak{c}}(h) \subset \tilde{\Omega}(h)$. It follows from Proposition 5.11 that the connected components of $\Omega(h) \backslash \Omega_{\mathfrak{c}}(h)$ coincide with the asymptotic regions defined above. Similarly for $\tilde{\Omega}(h) \backslash \tilde{\Omega}_{\mathfrak{c}}(h)$. 
The limit set $\Lambda$ is contained in the adherence of $\tilde{\Omega}_{\mathfrak{c}}(h)$ which is the union of a finite number of globally hyperbolic spacetimes. Hence there is a spacelike plane $P$ that does not intersect $\tilde{\Omega}_{\mathfrak{c}}(h)$, so that we can take the convex hull

$$
\tilde{\mathcal{K}}(h)
$$

of $\Lambda$ in $\mathbb{R}^{3}=\mathbb{P}^{3} \backslash \hat{P}$, where $\hat{P}$ is the projective plane containing $P$. It turns that $\tilde{\mathcal{K}}(h)$ is contained in the closure of $\tilde{\Omega}_{\mathfrak{c}}(h)$, it is $h$-invariant and does not depend on the choice of $P . \tilde{\mathcal{K}}(h)$ is called the convex core of $\tilde{\Omega}(h)$, as well as its quotient

$$
\mathcal{K}(h)
$$

is the convex core of $\Omega(h)$. We can see that $\tilde{\Omega}(h)$ coincides with the set of points in $\mathbb{X}_{-1}$ whose dual plane does not intersect $\tilde{\mathcal{K}}(h)$, and that every plane dual to some point of $\tilde{\mathcal{K}}(h)$ does not intersect $\tilde{\Omega}(h)$. The boundary of $\tilde{\mathcal{K}}(h)$ contains the spacelike geodesics of the horizons of $\tilde{\Omega}_{\mathfrak{c}}(h)$. Such geodesics disconnects $\partial \tilde{\mathcal{K}}(h)$ into two $h$ invariant pleated surfaces whose quotients are homeomorphic to $S$. One, say $\partial_{+} \tilde{\mathcal{K}}(h)$, is in the future of the other one, say $\partial_{-} \tilde{\mathcal{K}}(h)$, an they are called the future and the past boundary of $\tilde{\mathcal{K}}(h)$ respectively. It turns that $\partial_{+} \tilde{\mathcal{K}}(h)$ is obtained via the AdS bending of $\left(F_{+}, \lambda_{+}\right) \in \mathcal{M} \mathcal{L}_{\mathfrak{c}}(S)$ (according to Section 4) so that it is the future boundary of the past part of a specific $\mathcal{Y}\left(C_{+}\right) / h \in \mathcal{M G H}_{\mathfrak{c}}(h)$; in fact:

The extremal $h$-invariant meridian $C_{+}$is obtained by taking the lower extremal arc in each rectangle.

Similarly $\partial_{-} \tilde{\mathcal{K}}(h)$ is the past boundary of the future part of a specific spacetime $\mathcal{Y}\left(C_{-}\right) / h \in$ $\mathcal{M G H}_{\mathfrak{c}}(h)$ ( whose future boundary of the past part is obtained by bending a certain $\left.\left(F_{-}, \lambda_{-}\right) \in \mathcal{M} \mathcal{L}_{\mathfrak{c}}(S)\right)$. The corresponding extremal $h$-invariant meridian $C_{-}$is obtained by taking the upper extremal arc in each rectangle. This makes sense also when $\Omega(h)$ is globally hyperbolic; in such a case $C_{-}=C_{+}$.

Assume now that $\Omega(h)$ is not globally hyperbolic. For each boundary component $c_{i}$ of $F_{+}$, we have that $l_{c_{i}}$ is the size of the corresponding horizon, whereas $\mathrm{I}_{c_{i}}\left(\lambda_{+}\right)$is the corresponding momentum. It follows that $\lambda_{+}$belongs to the closure of $\mathcal{V}_{\mathfrak{c}}\left(F_{+}\right)$(recall Corollary 3.31). In fact this property uniquely characterizes $Y_{+}$within $\mathcal{M} \mathcal{G H}_{\mathfrak{c}}(h)$. In particular this selects a privileged one among the earthquakes of Theorem 3.33 . For $\left(F_{-}, \lambda_{-}\right)$we have the somehow opposite behaviour, that is for every boundary component $\mathrm{I}_{c_{i}}\left(\lambda_{-}\right)>l_{c_{i}}$.

Set

$$
\tilde{B}(h)=\mathcal{Y}\left(C_{-}\right), \quad B(h)=\tilde{B}(h) / h, \quad \tilde{W}(h)=\mathcal{Y}\left(C_{+}\right), \quad W(h)=\tilde{W}(h) / h .
$$

Denote by $K B(h), K W(h)$ the respective convex cores as MGH spacetimes. We have

Proposition 5.13. (1) $\mathcal{K}(h)=K B(h) \cap K W(h)$.

(2) $\tilde{\Omega}_{\mathfrak{c}}(h)=\tilde{B}(h) \cup \tilde{W}(h), \Omega_{\mathfrak{c}}(h)=B(h) \cup W(h)$.

In Physics literature the special globally hyperbolic spacetime $B(h)(W(h))$ is known as the multi black hole ( multi white hole) contained in the causal spacetime $\Omega(h)$. The attribute "multi" mostly refer to the fact that it has a "multi" horizon. $B(h)$ looks like a honest black hole in the sense that every future inextensible causal curve emanating from any event in $B(h)$ never leave $B(h)$ and eventually reach the final singularity 
$\Sigma_{+}$of $B(h)$ in finite time. In particular, lightlike rays emanating from $B(h)$ do not reach $\partial_{\infty} \Omega(h)$. So the final singularity $\Sigma_{+}$is an actual singularity for the spacetime $\Omega(h)$ itself, as it reflects its future timelike geodesic incompleteness ( at the initial singularity of $B(h)$ that is contained in its interior, $\Omega(h)$ is perfectly non singular). The initial singularity $\Sigma_{-}$of the white hole $W(h)$ plays a similar rôle with respect to the past. However, $\Sigma_{+}$is "censured" by the multihorizon of $B(h)$, while $\Sigma_{-}$is a "naked" singularity. In Figure 7 we see a schematic picture of $\Omega(h)$ with its convex core and its black hole.

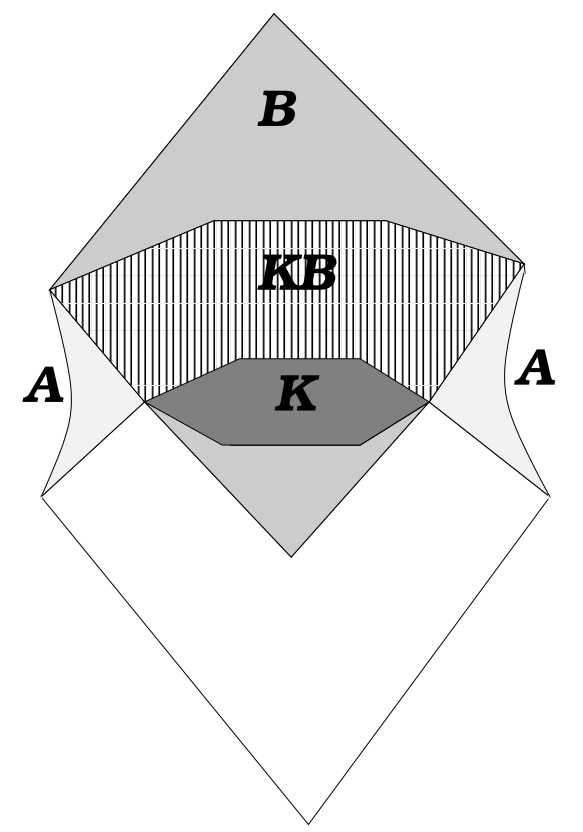

FiguRE 7. A schematic picture of $\Omega$ with its convex core $\mathcal{K}$ and asymptotic regions $\mathcal{A}$. It contains the black hole $\mathcal{B}$ with is convex core $\mathcal{K} \mathcal{B}$ containing $\mathcal{K}$.

Asymptotic regions and BTZ black holes. Every asymptotic region $A=A(\gamma)$ of $\Omega(h)$ has by itself a natural extension to a maximal causal AdS spacetime $\mathcal{B}=\mathcal{B}(\gamma)$, homeomorphic to $\left.\left(S^{1} \times \mathbb{R}\right) \times \mathbb{R}\right)$. $\mathcal{B}$ contains a maximal globally hyperbolic spacetime $\mathcal{B H}$, with a complete Cauchy surface homeomorphic to the annulus $S^{1} \times \mathbb{R}$, which is known as the BTZ black hole contained in $\mathcal{B}$ (see [5], [20]). $\mathcal{B}$ has been particularly studied because it supports Kerr-like metrics with several qualitative analogies with the classical rotating black hole solutions of $(3+1)$ gravity. Let us briefly recall this matter. It is convenient to lift $\mathbb{X}_{-1}=P S L(2, \mathbb{R})$ in $\hat{\mathbb{X}}_{-1}=S L(2, \mathbb{R})$ so that it is given by the matrices of the form

$$
X=\left(\begin{array}{cc}
T_{1}+X_{1} & T_{2}+X_{2} \\
-T_{2}+X_{2} & T_{1}-X_{1}
\end{array}\right)
$$

such that $\operatorname{det}(X)=1,0<T_{1}^{2}-X_{1}^{2}<1, X_{1}, T_{1}$ have a definite sign. We fix also a suitable $S L(2, \mathbb{R})$-lifting of the isometry $\left(h_{L}(\gamma), h_{R}(\gamma)\right)$ corresponding as above to the 
given asymptotic region. Let us assume for simplicity that it is of the form

$$
\left.\left(\begin{array}{cc}
\exp \left(r_{+}-r_{-}\right) & 0 \\
0 & \exp \left(r_{-}-r_{+}\right)
\end{array}\right),\left(\begin{array}{cc}
\exp \left(\left(r_{+}+r_{-}\right)\right) & 0 \\
0 & \exp \left(-\left(r_{+}+r_{-}\right)\right)
\end{array}\right)\right)
$$

and that $r_{+}>r_{-} \geq 0$. This isometry generates a group $\mathcal{G}$ that acts on the whole of $\hat{\mathbb{X}}_{-1}$, with a constant vector field $\xi$ as infinitesimal generator, and we have $q(\xi)=$ $\left(T_{2}^{2}-X_{2}^{2}\right) r_{+}+\left(T_{1}^{2}-X_{1}^{2}\right) r_{-}(q$ is defined in Section 2$)$. Roughly speaking $\tilde{\mathcal{B}}$ is the maximal region of $\mathbb{X}_{-1}$ such that:

(1) $q(\xi)>0$ on $\tilde{\mathcal{B}}$, so that we can take the function $r=q(\xi)^{1 / 2}>0$;

(2) $\left\{r_{+}>r>r_{-} \subset \tilde{\mathcal{B}}\right.$;

(3) $\tilde{\mathcal{B}}$ is $\mathcal{G}$-invariant, the group acts nicely and the quotient $\mathcal{B}$ is a causal spacetime homeomorphic to $\left(S^{1} \times \mathbb{R}\right) \times \mathbb{R}$.

$\tilde{\mathcal{B}}$ admits a $\mathcal{G}$-invariant "tiling" by regions of three types I, II, III contained in $\left\{r>r_{+}\right\}$, $\left\{r_{+}>r>r_{-}\right\},\left\{r_{-}>r\right\}$ respectively. Each region is bounded by suitable null horizons at which $r=r_{ \pm}$. We can see that our asymptotic regions $\tilde{A}$ are of type III.

By "joining" (the lifting of) the spacelike line $l_{\gamma}$ with the two liftings of the dual line $l_{\gamma}^{*}$ respectively, we get two "tetrahedra" say $\tilde{\mathcal{B}} \mathcal{H}$ and $\tilde{\mathcal{W}} \mathcal{H}$ embedded in $\tilde{\mathcal{B}}$ intersecting at $l_{\gamma}$. These are the two regions of type II that form the whole of $\left\{r_{+}>r>r_{-}\right\}$. One projects onto the BTZ black hole $\mathcal{B H}$, the other one covers the white hole embedded in $\mathcal{B}$, say $\mathcal{W H}$. Note that both $\tilde{\mathcal{B}} \mathcal{H}$ and $\mathcal{W H}$ are instances of "degenerate" globally hyperbolic spacetimes in the sense of Section 4.7 .

For suitable coordinates $(v, r, \phi)$ on $\mathcal{B}$, where $(r, \phi)$ look like polar coordinates on the $v$-level surfaces, the Kerr-like metric is of the form

$$
d s^{2}=\left(M-r^{2}\right) d v^{2}+f^{-1} d r^{2}+r^{2} d \phi^{2}-J d v d \phi
$$

where

$$
M=r_{+}^{2}+r_{-}^{2}, \quad J=2 r_{+} r_{-}, \quad M \geq J, \quad f=-M+r^{2}+\frac{J^{2}}{4 r^{2}}
$$

and they are related to the previously defined "size" and "momentum" by

$$
M+J=s^{2}, \quad M-J=m^{2} .
$$

Each region of $\mathcal{B}$ support this metric, the null horizons of the regions being just "coordinate singularities".

BTZ black holes naturally arise in the framework of Wick rotation-rescaling theory for the elementary surfaces of finite type, that is having Abelian fundamental group: $S=S^{1} \times \mathbb{R}$ and $S=S^{1} \times S^{1}$. This displays an interesting rôle of quadratic differentials instead of geodesic laminations. See Chapter 7 of [10] for more details.

5.5. (Broken) $T$-symmetry. Let $Y \in \mathcal{M G H}_{-1}(S)$. By reversing the time orientation we get onother spacetime $Y^{*} \in \mathcal{M G \mathcal { H }} \mathcal{H}_{-1}$. This involution is called T-symmetry as well the involution induced on $\mathcal{M L}(S)$ via the map $\mathfrak{m}_{-1}$. If $\mathcal{Y}(C)$ is the universal covering of $Y$, then the universal covering of $Y^{*}$ is $\mathcal{Y}^{*}=\mathcal{Y}\left(C^{*}\right)$, where $C^{*}$ is the image of the curve $C$ under the involution of $\partial \mathbb{X}_{-1}=S_{\infty}^{1} \times S_{\infty}^{1}$

$$
(x, y) \mapsto(y, x)
$$



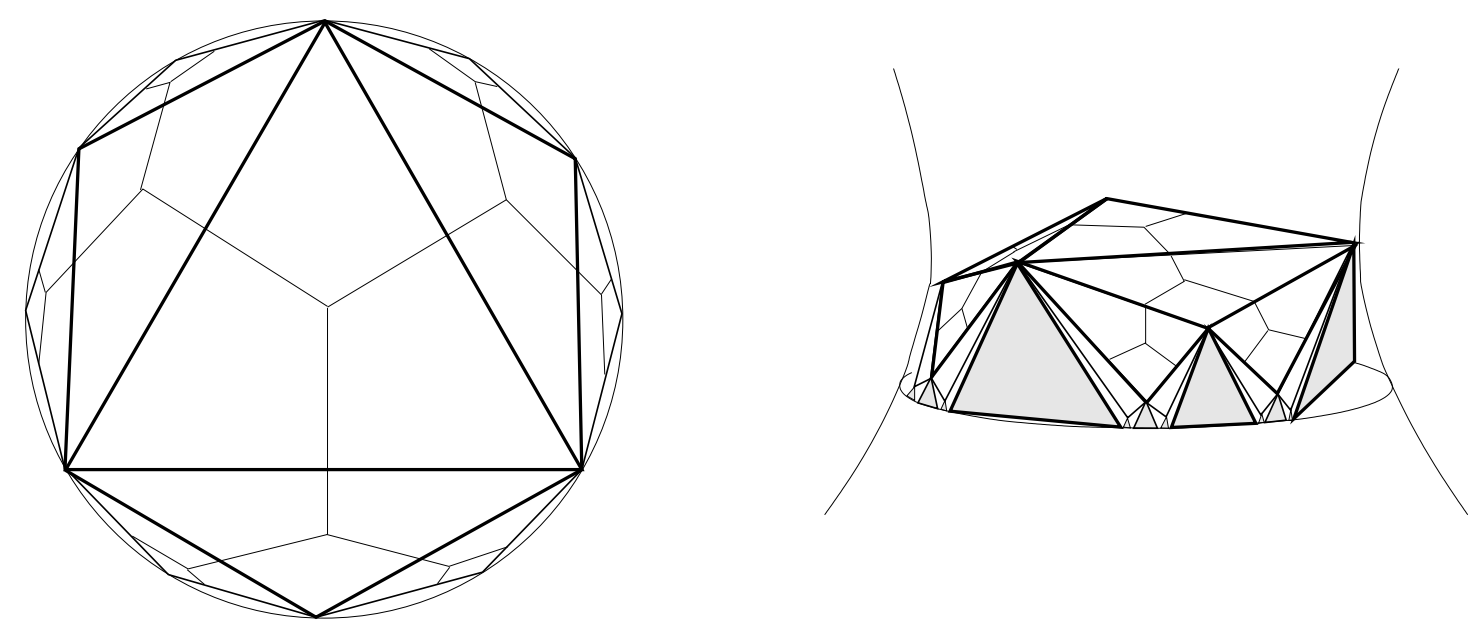

Figure 8 . The convex core of a black hole $B(h)$. On the left the lamination with its dual spine. On the right the bending of $\mathbb{H}^{2}$ along $\lambda$ in $\mathbb{X}_{-1}$. Grey regions are null components of the past boundary of $\mathcal{K}_{\lambda}$.

Moreover, the holonomy $h^{*}$ of $Y^{*}$ is obtained by exchanging the components of the holonomy of $h$ of $Y$

$$
h=\left(h_{-}, h_{+}\right) \leftrightarrow h^{*}=\left(h_{+}, h_{-}\right) .
$$

If $B(h)$ is the black hole of $\Omega(h)$, then $B(h)^{*}=W\left(h^{*}\right)$. The opposite behaviour "I $\mathrm{I}_{c}\left(\lambda_{+}\right) \leq l_{c}$ vs $\mathrm{I}_{c}\left(\lambda_{-}\right)>l_{c}$ " at the future boundary of the respective past parts (see above), can be considered as the basic feature of "broken $T$-symmetry". A particular instance is when $B(h)$ is encoded by $(F, \lambda)$ such that $F \in \mathcal{T}_{g, r}=\mathcal{T}(S) \cap \mathcal{T}_{\mathfrak{c}}(S)$ (the smallest stratum of $\mathcal{T}_{\mathfrak{c}}(S)$ ), and $\lambda$ enters the cusps (in the Figure we show an example of $B(h)$ where $F$ has $g=0, r=3$, and the lamination is like in Example 3.15 with respect to a standard ideal triangulation of $F$ by two triangles). In this case $F^{*}$ belongs indeed to a higher dimensional cell of $\mathcal{T}_{\mathfrak{c}}(S)$ and the white hole $W\left(h^{*}\right)$ has the property that $\mathrm{I}_{\lambda^{*}}(c)=l(c)$ at every boundary curve, and every asymptotic region has null momentum.

\section{INCLUDING PARTICLES}

In 3-dimensional gravity massive point particles can be modeled as cone singularities along timelike lines. In particular, the rest mass $m$ of a particle is related to the curvature $k$ concentrated along its timelike geodesic "world line" by

$$
k=2 \pi m, \quad k=2 \pi-\alpha
$$

where $\alpha$ is the cone angle. If we require that the mass is positive, then it is bounded by $0 \leq m \leq 1$, while $2 \pi \geq \alpha \geq 0$. However, there are no real geometric reasons to exclude cone angles bigger than $2 \pi$.

It is a natural question whether Wick Rotation-rescaling theory does apply also on cone spacetimes. In such a perspective, it is quite natural to extend the space $\mathcal{T}_{\mathfrak{c}}(S)$ defined in Section 3 , by extending the notion of "type" to $\theta=V_{\mathcal{H}} \cup V_{C}$, and allowing hyperbolic structures $F$ on $S$ whose completion $F^{\mathcal{C}}$ is compact, as usual has geodesic boundary components corresponding to the points of $V_{\mathcal{H}}$, and possibly has conical singularities at the points of $V_{C}$. In particular we allow also holonomy of elliptic type 
at the circles surrounding these points. The parabolic holonomies correspond now to cone angles equal to 0 , hence to particles of extremal mass. In order to preserve the conical structure, we can consider measured geodesic laminations on such cone surfaces $F$ that have compact support $L$ in $F$, that is whose closure in $F^{\mathcal{C}}$ does not intersect the singularities.

For the sake of simplicity (and following [18] to which we will refer for most results stated in this Section), from now on we will consider the particular case such that $V_{\mathcal{H}}=\emptyset$. Not even in this simplest case a complete answer to the above question is known. Only a few partial results are known, mostly concerning the case of "small" cone angles $(<\pi)$, or equivalently the case of particles with "big" masses.

Let $\hat{S}, S=\hat{S} \backslash V, V=\left\{p_{1}, \ldots, p_{r}\right\}$ be as usual. Let $g$ be the genus of $\hat{S}$. Fix an $r$-tuple of angles $\mathcal{A}=\left(\alpha_{1}, \ldots, \alpha_{r}\right)$, such that the "Gauss-Bonnet inequality"

$$
\sum_{j}\left(1-\frac{\alpha_{j}}{2 \pi}\right)>2-2 g
$$

holds; notice that we are not requiring here that the cone angles are smaller than $2 \pi$. We denote by

$$
\mathcal{T}_{\mathfrak{c}}(S, \mathcal{A})
$$

the Theichmüller space of hyperbolic structures $F$ on $S$ whose completion $F^{\mathcal{C}}$ has conical singularities at $p_{1}, \ldots, p_{r}$, of cone angles $\alpha_{1}, \ldots, \alpha_{r}$.

By a general result of Troyanov [51, we have

Proposition 6.1. The natural map

$$
\mathcal{T}_{\mathfrak{c}}(S, \mathcal{A}) \rightarrow \mathcal{T}_{g, r}
$$

that associates to every $F \in \mathcal{T}_{\mathfrak{c}}(S, \mathcal{A})$ the unique complete hyperbolic structure of finite area $\tilde{F}$ on $S$ in the same conformal class of $F$, is a bijection.

This means in particular that $\mathcal{T}_{\mathfrak{c}}(S, \mathcal{A})$ is not empty. If $\alpha_{j}=0$ for every $j$, then $\tilde{F}=F$ and $\mathcal{T}_{\mathfrak{c}}(S, 0)$ just coincides with $\mathcal{T}_{g, r}$.

For every $F \in \mathcal{T}_{\mathfrak{c}}(S, \mathcal{A})$, we denote by $\mathcal{M L}_{\mathfrak{c}}(F, \mathcal{A})$ the space of measured geodesic laminations on $F$ with compact support. The space of all such $(F, \lambda)$ 's is denoted by $\mathcal{M L}_{\mathfrak{c}}(S, \mathcal{A})$. When $\mathcal{A}=0$, then $\mathcal{M L}_{\mathfrak{c}}(F, 0)$ just coincides with $\mathcal{M} \mathcal{L}_{\mathfrak{c}}(\tilde{F})^{0}$ (defined in Section [3). More generally we have:

Proposition 6.2. Assume that for every $j, \alpha_{j}<\pi$. Then there is a natural identification between $\mathcal{M L}_{\mathfrak{c}}(F, \mathcal{A})$ and $\mathcal{M L}_{\mathfrak{c}}(\tilde{F})^{0}$.

We give a brief sketch of the proof of this proposition, assuming some familiarity with "train-tracks". Since $\alpha_{i}<\pi$, for small $\epsilon>0$, the complement, say $\Sigma_{\epsilon}$, of a regular neighborhood of $V$ in $F^{\mathcal{C}}$ of ray $\epsilon$ is convex. As a consequence, any non-peripheral loop on $F$ admits a geodesic representative whose distance from $V$ is at least $\epsilon$.

Given $\tilde{\lambda} \in \mathcal{M L}_{\mathfrak{c}}(\tilde{F})^{0}$, its support is contained in $\Sigma_{\epsilon}$, for $\epsilon$ sufficiently small. Since $\Sigma_{\epsilon}$ is compact, it follows that the leaves of $\tilde{\lambda}$ are quasi-geodesic in $F$. Since $\Sigma_{\epsilon}$ is convex, they can be stretched to become geodesic with respect to $F$. The union of all these leaves makes a geodesic lamination $\lambda$ on $F$. A train-track carrying $\tilde{\lambda}$ carries also $\lambda$ so 
this last can be equipped with a transverse measure corresponding to the measure on $\tilde{\lambda}$.

Remark 6.3. If some cone angle $\alpha_{i}$ is bigger than $\pi$, then Proposition 6.2 fails. In fact it is not difficult to construct a surface with cone angles bigger than $\pi$ and a loop $c$ whose geodesic representative passes through the singular point.

6.1. Maximal globally hyperbolic spacetimes with particles. Since the causal structure of a spacetime with timelike geodesic world lines of conical singularities extends also on the singular locus, we can extend as well the notion of Cauchy surfaces. These turn to be spacelike with conical singularities. Such a cone spacetime is said globally hyperbolic if it contains a Cauchy surface. Similarly to the smooth case, we can restrict our study to maximal globally hyperbolic ones. More precisely we set

$$
\mathcal{M G H}_{\kappa}(S, \mathcal{A})
$$

the Teichmüller-like space of cone spacetime structures $h$ on $\hat{S} \times \mathbb{R}$, of constant curvature $\kappa \in\{-1,0,1\}$, such that:

- $h$ is non singular on $S \times \mathbb{R}$;

- $h$ has a timelike geodesic line of conical singularity of angle $\alpha_{i}$ at each $\left\{p_{i}\right\} \times \mathbb{R}$;

- $h$ is a maximal globally hyperbolic and has a Cauchy surface orthogonal to the singular set;

- these structures are considered up to isotopies of $\hat{S} \times \mathbb{R}$ preserving $V \times \mathbb{R}$.

By easily adapting the constructions of Section 4 we get, for every $\kappa=0, \pm 1$, the map

$$
\mathfrak{m}_{\kappa}: \mathcal{M L}_{\mathfrak{c}}(S, \mathcal{A}) \rightarrow \mathcal{M G \mathcal { H }}{ }_{\kappa}(S, \mathcal{A})
$$

The main differences are that even for $\kappa=0,-1$ the developing maps are no longer embeddings; moreover, also the asymptotic complex projective structures produced by the Wick rotations have conical singularities. As in the smooth case, the maps $\mathfrak{m}_{\kappa}$ are injective. For the so obtained spacetimes have cosmological time, and one can recover the corresponding data $(F, \lambda)$ by looking at the level surfaces of the cosmological time. Moreover, by construction, canonical Wick rotations and rescalings, directed by the gradient of the cosmological times, with the usual universal rescaling functions, apply to the spacetimes belonging to the images of the maps $\mathfrak{m}_{\kappa}$.

On the other hand, the question of having an intrinsic characterization of the images $\operatorname{Im}\left(\mathfrak{m}_{\kappa}\right)$ is largely open. In particular one asks to determine, for every $\kappa$, the angle assignments $\mathcal{A}$ such that $\mathfrak{m}_{\kappa}$ gives a parametrization of the whole of $\mathcal{M G H}_{\kappa}(S, \mathcal{A})$ (possibly inverting the time orientation). We have (see [18])

Proposition 6.4. If all the cone angles are less than $\pi$, then the spacetimes belonging to $\operatorname{Im}\left(\mathfrak{m}_{-1}\right)$ are precisely those admitting a convex Cauchy surface orthogonal to the singular locus.

In fact, under such a "big masses" hypothesis, being in the image of $\mathfrak{m}_{-1}$ turns to be equivalent to admit a convex core, that is a minimal convex subset. The convex core is homeomorphic to $\hat{S} \times \mathbb{R}$ and its boundary is the union of two $\mathrm{C}^{0,1}$-spacelike, intrinsically hyperbolic bent cone surfaces $\partial_{+} \mathcal{K}(Y)$ and $\partial_{-} \mathcal{K}(Y)$, orthogonal to the singular locus. Just like the non-singular case, $Y$ is encoded by $(F, \lambda)$ iff the future boundary of its 
convex core is obtained by bending $F$ along $\lambda$; similarly for the past boundary, via $T$-symmetry.

One would expect that for big masses, the map $\mathfrak{m}_{-1}$ actually is a bijection, that is a convex Cauchy surface should always exist.

If some cone angle is bigger or equal than $\pi$, it is known that in general the maps $\mathfrak{m}_{\kappa}$ are not onto, even if all masses are positive. For example in [11](2), by applying a so called "patchwork" construction, one produces flat MGH cone spacetimes with positive masses and with some cone angles equal to $\pi$, that do not belong to the image of $\mathfrak{m}_{0}$. In fact it is remarkable that these spacetimes have nevertheless cosmological time whose level surfaces are orthogonal to the singular locus, and are flat instead of hyperbolic at the singular points of cone angle $\pi$. The canonical rescalings apply to them so that we finally get also spacetimes that do not belong to the images of $\mathfrak{m}_{ \pm 1}$.

6.2. Earthquakes on hyperbolic cone surfaces. As every lamination $\lambda \in \mathcal{M} \mathcal{L}_{\mathfrak{c}}(F, \mathcal{A})$ avoids the conical points, the notion of earthquake along such a lamination is defined as well. Similarly to the non-singular case, we have (see [18])

Theorem 6.5. If $Y$ has big masses, belongs to $\operatorname{Im}\left(\mathfrak{m}_{-1}\right)$, and is encoded by $(F, \lambda) \in$ $\mathcal{M L}_{\mathfrak{c}}(S, \mathcal{A})$, then the left (resp. right) earthquake on $F$ along $\lambda$ produces surfaces $\beta_{L}(F, \lambda)$ (resp. $\left.\beta_{R}(F, \lambda)\right) \in \mathcal{T}_{\mathfrak{c}}(S, \mathcal{A})$ whose holonomy coincides with the right (resp. left) holonomy of $Y$.

Under the big masses hypothesis, let us consider the map

$$
\mu: \operatorname{Im}\left(\mathfrak{m}_{-1}\right) \rightarrow \mathcal{T}_{\mathfrak{c}}(S, \mathcal{A}) \times \mathcal{T}_{\mathfrak{c}}(S, \mathcal{A})
$$

that associates to every $Y$ the points obtained by left and right earthquake on $(F, \lambda)$ respectively, as above. We have ([18])

Theorem 6.6. The following equivalent facts hold

(1) Given $F, F^{\prime} \in \mathcal{T}_{\mathfrak{c}}(S, \mathcal{A})$ there exists a unique $\lambda \in \mathcal{M L}_{\mathfrak{c}}(F, \mathcal{A})$ such that $\beta_{L}(F, \lambda)=$ $F^{\prime}$.

(2) The map $\mu$ is bijective.

Notice that the first statement is in purely hyperbolic terms. The equivalence between the two statements follows from Theorem 6.5. This equivalence between the hyperbolic and Lorentzian formulations plays an subtle rôle in the proof of Theorem 6.6. In fact by means of the hyperbolic formulation the map $\mu$ is proved to be locally injective, whereas Lorentzian geometry is used to prove that it is a proper map.

Finally we mention that in Chapter 7 of [10], we have described a quite different family of spacetimes with cone angles $\geq \pi$ (i.e. possibly with negative masses) that are governed by quadratic differentials rather than by measured geodesic laminations, and such that Wick rotation-rescaling machinery does apply to them.

\section{REFERENCES}

[1] W. Abikoff, The real analytic theory of Teichmüller space; Springer LNM 820 (1980);

[2] L. Andersson, G.J. Galloway and R. Howards, The cosmological time function, Class. Quantum Grav. 15 (1998) 309-322;

[3] L. Andersson, V. Moncrief, A.J. Tromba, On the global evolution problem in $2+1$ gravity, J. Geom. Phys. 23 (1997), no. 3-4, 191-205; 
[4] B. Apanasov, The geometry of Nielsen's hull for a Kleinian group in space of quasi-conformal mappings, Ann.Glob.Anal.Geom. 6 (1998) 207-230;

[5] M. Banados, M. Henneaux, C. Teitelboim, J. Zanelli, Geometry of the 2+1 black hole, Physical Review D, 48, (1993);

[6] - (1) T. Barbot, Globally hyperbolic flat spacetimes, Journ. Geom. Phys. 53 (2005), 123-165;

- (2) Causal properties of AdS-isometry groups I: Causal actions and limit sets, arXiv: math.GT/0509552;

- (3) Causal properties of AdS-isometry groups II: BTZ multi black-holes, arXiv: math.GT/0510065;

[7] T. Barbot, G. Zeghib, F. Beguin Constant mean curvature foliations of globally hyperbolic spacetimes locally modelled on $A d S_{3}$; arXiv math.MG/0412111;

[8] (1) S. Baseilhac, R. Benedetti, Quantum hyperbolic invariants of 3-manifolds with PSL $(2, \mathbb{C})$ characters, Topology, 43 Issue 6, (2004), 1373-1423;

- (2)Classical and Quantum Dilogarithmic Invariants of 3-Manifolds with Flat PSL(2, C)-bundles, Geometry and Topology, 9, 2005;

- (3) Quantum hyperbolic geometry, arXiv math.GT/0611504, to appear in Algebraic and Geometric Topology;

[9] J.T. Beem, P.E. Ehrlich, K.L. Easley, Global Lorentzian Geometry, 2nd edn., Pure and Applied Mathematics, Vol. 202. Dekker ;

[10] R. Benedetti, F. Bonsante, Canonical Wick rotations in 3-dimensional gravity, arXiv math. math.DG/0508485, v3 25 October 2006, to appear in Memoirs A.M.S;

[11] (1) R. Benedetti and E. Guadagnini, Cosmological time in (2+1)-gravity, Nuclear Phys. B 613 (2001), 330-352;

- (2) Geometric cone surfaces and (2+1)-gravity coupled to particles, Nuclear Phys. B 588 (2000), no. 1-2, 436-450;

- (3) Classical Teichmüller theory and (2+1) gravity, Physics Letters B 441 (1998) 60-68;

[12] R. Benedetti, C. Petronio, Lectures on Hyperbolic Geometry, Universitext, Springer 1992;

[13] L. Bers, Uniformization by Beltrami equations, Comm. Pure Appl. Math., 14(1961), 215-228;

[14] - (1) F. Bonahon, Geodesic laminations on surfaces, Laminations and foliations in dynamics, geometry and topology (Stony Brook, NY, 1998), 1-37, Contemp. Math., 269, Amer. Math. Soc., Providence, RI, 2001;

- (2)Kleinian groups which are almost Fuchsian, J.Reine Angew. Math, 587 (2005), 1-15;

- (3) The geometry of Teichmüller space via geodesic currents, Invent. Math 92 (1988), 139-162;

- (4) Shearing hyperbolic surfaces, bending pleated surfaces and Thurston's symplectic form, Ann. Fac. Sc. Toulouse Math. 5 (1996) 233-297;

[15] F. Bonahon and J-P. Otal, Laminations mesurées de plissage des varietés hyperboliques de dimension 3, Ann. of Math, 160 (2004), 1013-1055;

[16] F. Bonsante, PhD Thesis, Scuola Normale Superiore, Pisa (2005);

[17] F. Bonsante, Flat Spacetimes with Compact Hyperbolic Cauchy Surfaces, Journ. Diff. Geom. 69, (2005), 441-521;

[18] F. Bonsante, J.M. Schlenker, AdS manifolds with particles and earthquakes on singular surfaces, arXiv: math.GT/0609116;

[19] F. Bonsante, K. Krasnov, J.M. Schlenker, Multi black holes and earthquakes on Riemann surfaces with boundaries, in preparation;

[20] S. Carlip, The $(2+1)$-dimensional black hole, Classical Quantum Gravity 12 (1995), no. 12, 2853-2879;

[21] S. Carlip, Quantum gravity in $2+1$ dimensions, Cambridge Monographs on Mathematical Physics. Cambridge University Press, Cambridge, 1998;

[22] Y. Choquet-Bruhat, R.Geroch. Global aspects of the Cauchy problem in general relativity, Comm. Math. Phys., 14 (1969), 329-335;

[23] N. Dunfield, Cyclic surgery, degree of maps of character curves, and volume rigidity for hyperbolic manifolds, Inventiones Mathematicae 136 (1999), 623-657; 
[24] D.B.A. Epstein and A. Marden, Convex hulls in hyperbolic space, a theorem of Sullivan and measured pleated surfaces, London Math. Soc. Lectures Note Ser., Cambridge University Press 111 (1987), 113-253;

[25] A. Fathi, F. Laudenbach, V. Poenaru, Travaux de Thurston sur les surfaces, Astérisque 66-67 (1979);

[26] V.V. Fock, A.B. Goncharov, Dual Teichmuller and lamination spaces, In Handbook on Teichmüller theory (A. Papadopoulos, ed.), Volume I, EMS Publishing House, Zürich 2007;

[27] G.W. Gibbons, J.B. Hartle, Real tunneling geometries and the large-scale topology of the universe, Phys. Rev. D (3) 42 (1990), no. 8, 2458-2468;

[28] G.W. Gibbons, Real tunnelling geometries, Topology of the Universe Conference (Cleveland, OH, 1997). Classical Quantum Gravity 15 (1998), no. 9, 2605-2612;

[29] (1) W.M. Goldman, Projective structures with Fuchsian holonomy, J. Differential Geom. 25 (1987) 297-326; 136, (1999), 623-657

- (2) The Margulis invariant of isometric actions of Minkowsky (2+1)-space in "Rigidity in Dynamics and geometry (Cambridge 2000)" 187-201, Springer 2002;

[30] W.M. Goldman, G.A. Margulis, Flat Lorentz 3-manifolds and cocompact Fuchsian groups, Contemp. Math. 262 (2000), 135-145.

[31] S. Hawking, G. Ellis, The large scale structure of space-time, Cambridge University Press 1978;

[32] M. Kapovich, Hyperbolic manifolds and discrete groups, Progress in Mathematics 183, Birkhäuser (2001);

[33] R.M. Kashaev, A link invariant from quantum dilogarithm, Modern Phys. Lett. A 10 (1995) 1409-1418

[34] R. M. Kashaev, The hyperbolic volume of knots from the quantum dilogarithm, Lett. Math. Phys. 39 (1997), 269-275

[35] S.P. Kerckhoff, The asymptotic geometry of Teichmüller space, Topology, 19, N. 1 (1980);

[36] K. Krasnov, J.M. Schlenker, Minimal surfaces and particles in 3-manifolds, arXiv: math.DG/0511441;

[37] R.S. Kulkarni, U. Pinkall, A canonical metric for Möbius structures and its applications, Math. Z. 216 (1994) n. 1, 89-129;

[38] G.A. Margulis, Complete affine locally flat manifolds with free fundamental group, J. Soviet Math 134 (1987) 129-134;

[39] H-J. Matschull, The phase space structure of multi-particle models in $2+1$ gravity, Classical Quantum Gravity 18 (2001), no. 17, 3497-3560;

[40] C. Mc Mullen, Complex eartquakes and Teichmüller theory, J. Amer. Math. Soc. 11 (1998) 283320 ;

[41] G. Mess, Lorentz Spacetimes of Constant Curvature, Preprint IHES/M/90/28, Avril 1990;

[42] V. Moncrief, Reduction of the Einstein equations in $2+1$ dimensions to a Hamiltonian system over Teichmüller space, J. Math. Phys. 30 (1989), no. 12, 2907-2914;

[43] J-P. Otal, The hyperbolization theorem for fibered 3-manifolds, SMF/AMS Texts and Monographs, 7. American Mathematical Society, Providence, RI; Société Mathématique de France, Paris, 2001;

[44] R.C. Penner with J.L. Harer, Combinatorics of Train Tracks, Princeton University Press, 1992;

[45] Scannell, Flat conformal structures and classification of de Sitter manifolds, Comm.Anal.Geom. 7 (1999) 283-320;

[46] H. Masur, J. Smillie Quadratic differentials with prescribed singularities and pseudo-Anosov diffeomorphisms, Comment. Math. Helv. 68 (1993), no. 2, 289-307;

[47] (1) G. 't Hooft, Causality in (2+1)-dimensional gravity, Classical Quantum Gravity 9 (1992), no. $5,1335-1348$;

- (2) G. 't Hooft, Nonperturbative 2 particle scattering amplitudes in $2+1$-dimensional quantum gravity, Comm. Math. Phys. 117 (1988), no. 4, 685-700;

- (3) S. Deser, R. Jackiw, G. 't Hooft, Three-dimensional Einstein gravity: dynamics of flat space, Ann. Physics 152 (1984), no. 1, 220-235;

[48] J.G. Ratcliffe, Foundations of hyperbolic manifolds, Graduate Text in Math., Springer (1994); 
[49] W.P. Thurston, Geometry and topology of 3-manifolds, Electronic Version 1.0 Octber 1997 http://www.msri.org/gt3m/;

[50] W.P. Thurston, Earthquakes in two-dimensional hyperbolic geometry, in Analytical and Geometric Aspects of Hyperbolic Space, D.B.A. Epstein ed. London Math. Soc. Lectures Notes 111, Cambridge University Press, London 1987;

[51] M. Troyanov, Prescribing curvature on compact surfaces with cone singularities, Trans. Amer. Math. Soc., 324(1991), 793-821;

[52] (1) E. Witten, $2+1$-dimensional gravity as an exactly soluble system, Nuclear Phys. B 311 (1988/89), no. 1, 46-78;

- (2) Topology-changing amplitudes in $(2+1)$-dimensional gravity, Nuclear Phys. B 323 (1989), no. 1, 113-140. 